\title{
RESILIENT INFRASTRUCTURE: \\ REDEFINED
}

IMPROVING WATER INFRASTRUCTURE IN THE WAIRARAPA 



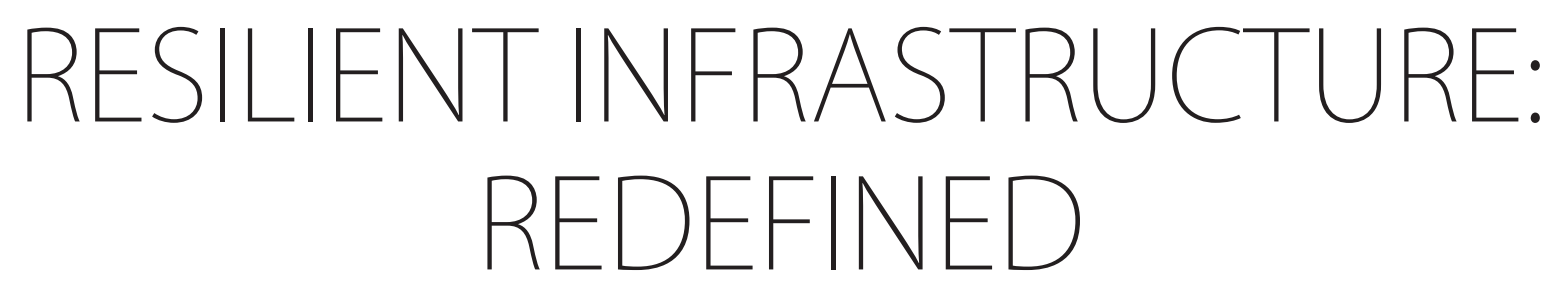

IMPROVING WATER INFRASTRUCTURE IN THE WAIRARAPA

A 120 Point thesis submitted in partial fulfilment of the requirements of Master of Landscape Architecture Victoria University of Wellington, School of Architecture 2016

\section{Kurt Cole}

Victoria University of Wellington, 2016

School of Architecture 



\section{ACKNOWLEDGEMENTS}

The completion of my master degree would not have been possible without the infinite support offered to me by many people, whether or not they are aware of how much I have appreciated it over the last five years.

First and foremost I must thank Bruno Marques, my supervisor and friend. Your perseverance, guidance and encouragement is the reason I am completing this Master's degree with the utmost pride in my work. Your commitment and endless help has provided me with the inspiration needed to complete this research.

To my fellow classmates, words can not express how much I will forever be in your debt. To the best bunch of people that I have shared the past five years with, thank you. In particular I must thank my classmates, flatmates and best friends, Megan and Grachia. I could not think of two better people to spend so, so many hours with, its been the best.

I must also make a special mention to the Wellington Regional Council for providing the 'Wairarapa Group' with funding to ensure many field trips to the Wairarapa were possible. To the Wairarapa Moana Management Team your support also was much appreciated giving invaluable feedback and endless knowledge.

Thanks to Ra Smith for his endless time and energy invested in this project, and those previously completed. The privilege to have access to such an extensive knowledge bank of the Wairarapa, alongside continued enthusiasm for our work, has driven us all to reach this final point in our studies, kia ora.

Finally I owe the biggest thanks to my family, for their endless support throughout my studies and always providing me with the encouragement that I needed to push on through. Understanding when I could not make special events due to my studies, listening to me when I was ready to give up and laughing with me when I needed some humour. You guys are the best. 



\section{ABSTRACT}

The abundance of natural environments within New Zealand is under threat, and unhindered profit-driven development is ever increasing, putting our landscapes at risk. A weak relationship with our land is currently resulting in detrimental development. In particular, designed infrastructure is often imposed on the landscape with little consideration for the effects it has on wider ecological systems. The degradation of our natural environment is spiralling out of control, landscape architecture has the potential to protect and enhance our natural environment through integrated design that benefits our natural systems and the people who exist within them.

This research aims to mitigate the adverse effects development has on the landscape through the use of naturally integrated water treatment infrastructure design. The cleansing abilities of natural wetlands are currently overlooked as precedents for design. An opportunity lies within the integration of natural wetlands and infrastructure, the outcome being new multipurpose landscapes. The fusion of water infrastructure and natural systems has the potential to not only mitigate adverse effects of current development, but also provide the public with diverse open spaces that support recreation and natural amenity.

Wairarapa Moana in the South Wairarapa is the site for this design research to take place. The abundance of public open spaces surrounding Lake Waiararapa, paired with the severe degradation of the water quality provide an opportunity for design research to explore possible solutions. The intention of this work is to diminish the harmful effects of development and poor land use in the area, resulting in the creation of natural spaces that have an underlying function of water treatment and fitting seamlessly into the wider ecological systems of the site. The space will also cater for various recreational activities, providing the South Wairarapa with a new typology of landscape that is resilient and responsive to the natural flux of this unique lake system. 


\section{CONTENTS}

\subsection{INTRODUCTION}

1.1 THE SITUATION 03

1.2 THE WAIRARAPA SCOPE 07

1.3 WAIRARAPA MOANA IN CONTEXT 09

1.4 LITERATURE REVIEW 10

1.4.1 LANDSCAPE FROM THE BASE 10

1.4.2 ENVIRONMENTAL INFRASTRUCTURE 12

1.4.3 CULTURE IDENTIFIED 15

\subsection{APPROACH}

2.1 METHODOLOGY 21

$\begin{array}{lll}2.2 & \text { SITE ANALYSIS } & 23\end{array}$

2.2.1 SOUTH WAIRARAPA SITUATION 23

$\begin{array}{lll}\text { 2.2.2 BUILTINFRASTRUCTURE } & 27\end{array}$

$\begin{array}{lll}2.2 .3 & \text { HYDROLOGY } & 29\end{array}$

2.2.4 VEGETATION 31 
2.2.5 LANDSCAPE GOVERNANCE 33

2.2.6 WAIRARAPA MOANA 35

2.2.7 CONCLUSIONS

\subsection{ANALYSIS}

3.1 PRECEDENT ANALYSIS 41

3.1.1 AVONHEAD RIVER 43

3.1.2 BRICK PITRING WALK

3.1.3 NATIONAL TOURISTROUTE 51

3.2 PROGRAMME ANALYSIS

3.2.1 DETAIL SITE 1

3.2.2 DETAIL SITE 2

3.2.3 DETAIL SITE $3 \quad 61$

3.2.4 REGIONAL CONCLUSIONS 63 


\subsection{DESIGN}

4.1 DESIGNED RESILIENCE 67

4.2 PRELIMINARY DESIGN 69

4.2.1 DESIGNTESTING 1

4.2.2 DESIGN TESTING 2 75

4.2.3 DESIGN TESTING 3

4.2.4 REGIONAL SITE APPLICATION 83

4.3 RESILIENT LANDSCAPES 85

4.3.1 FUNCTIONING HYDROLOGY 87

4.3.2 DESIGNING FOR FLUX 97

$\begin{array}{lll}\text { 4.3.3 ECONOMICAL LANDSCAPES } & 107\end{array}$

4.3.4 RECREATIONAL INFRASTRUCTURE 113

4.3.5 AMENITYVIEWS 125

4.3.6 THE SEASONAL SITE 133 
5.0 DISCUSSION

5.1 A KINSHIP WITH THE LAND 141

$\begin{array}{lll}5.3 & \text { FINAL FINDINGS } & 144\end{array}$

$\begin{array}{lll}6.0 & \text { BIBLIOGRAPHY } & 146\end{array}$

$\begin{array}{lll}7.0 & \text { LIST OF FIGURES } & 148\end{array}$

$\begin{array}{lll}8.0 & \text { APPENDIX } & 150\end{array}$ 




\subsection{THE SITUATION}

New Zealand's landscape is an invaluable resource that is often taken for granted and undervalued in today's economy. Providing economic benefits alongside cultural identity, the natural landscape is fast diminishing as the population expands and development sprawls. Currently development is driven by economic benefits, often having adverse effects on New Zealand's landscape. A shift in thinking is required to ensure the natural environment is protected for the future, a shift that sees the amenity values of the land having equal benefit to the economic return of development. A unique opportunity to regain some of the natural landscape values through design is present within this country, harnessing the relatively short time that has passed since harmful development began to effect the natural systems.

Development has been harsh on New Zealand's landscape, driven by economics to ensure developers get maximum return on their investment, even if this proves detrimental to our landscape. Modern relationships with the land lack respect and consideration for the adverse effects that development takes on the environment. This thesis argues that a relationship between the New Zealand people and their landscape can be altered through design. A shift from a European influence that currently sees a negative relationship that is driven by economy. Value will be restored to the land through the new formed bond, something that has not been present since pre European Maori dominated the landscape, taking from it only what they needed to survive. The value returned to the landscape as a crucial natural resource will therefore positively inform design to achieve resilient spaces for the unpredictable future.

Protecting natural public space within New Zealand is becoming tougher as the economic viability to develop such space increases, overshadowing amenity value. Exploring how this threatened natural land can be used through design to create landscapes that not only provide amenity value but also begin to mitigate the adverse effects that inevitable development has. Creating a catalyst for the future, the landscape will be designed in such 


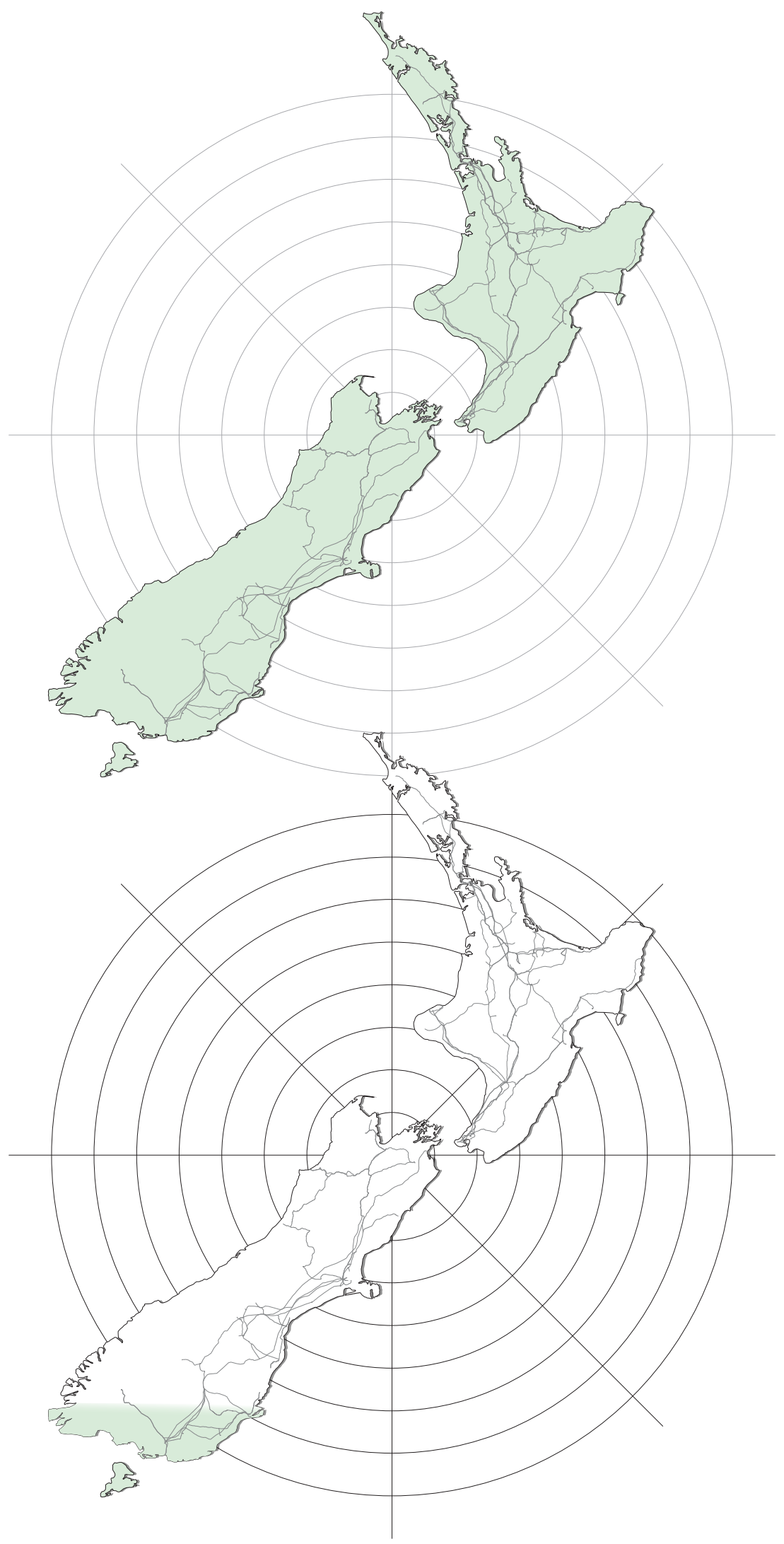


a way that it supports public users with an underlying ecological function that benefits the environment. An opportunity design for the environment in a positive way could lead landscape architecture into the forefront of infrastructure design.

The design of public space has many complexities in both theory and practicality, the outcome needs to be both successful in supporting a vibrant public ecology but also economically viable as little funding is commonly available. New Zealand's landscape is unique, natural systems stripped from the land in order to make it more productive are long lost. (Fig 1.0) Landscape architecture will be used to explore how much of this past natural system is suitable for restoration to lessen the effects of current heavy water infrastructure. To ensure the natural system that once dominated the landscape have the opportunity to be restored successfully, water treatment infrastructure will be integrated.
Fig 1.0 Wetland Decline Diagram (Left)

Diagram expressing the $90 \%$ area decline of New Zealand's indigenous wetland ecosystems over the past 150 years. 

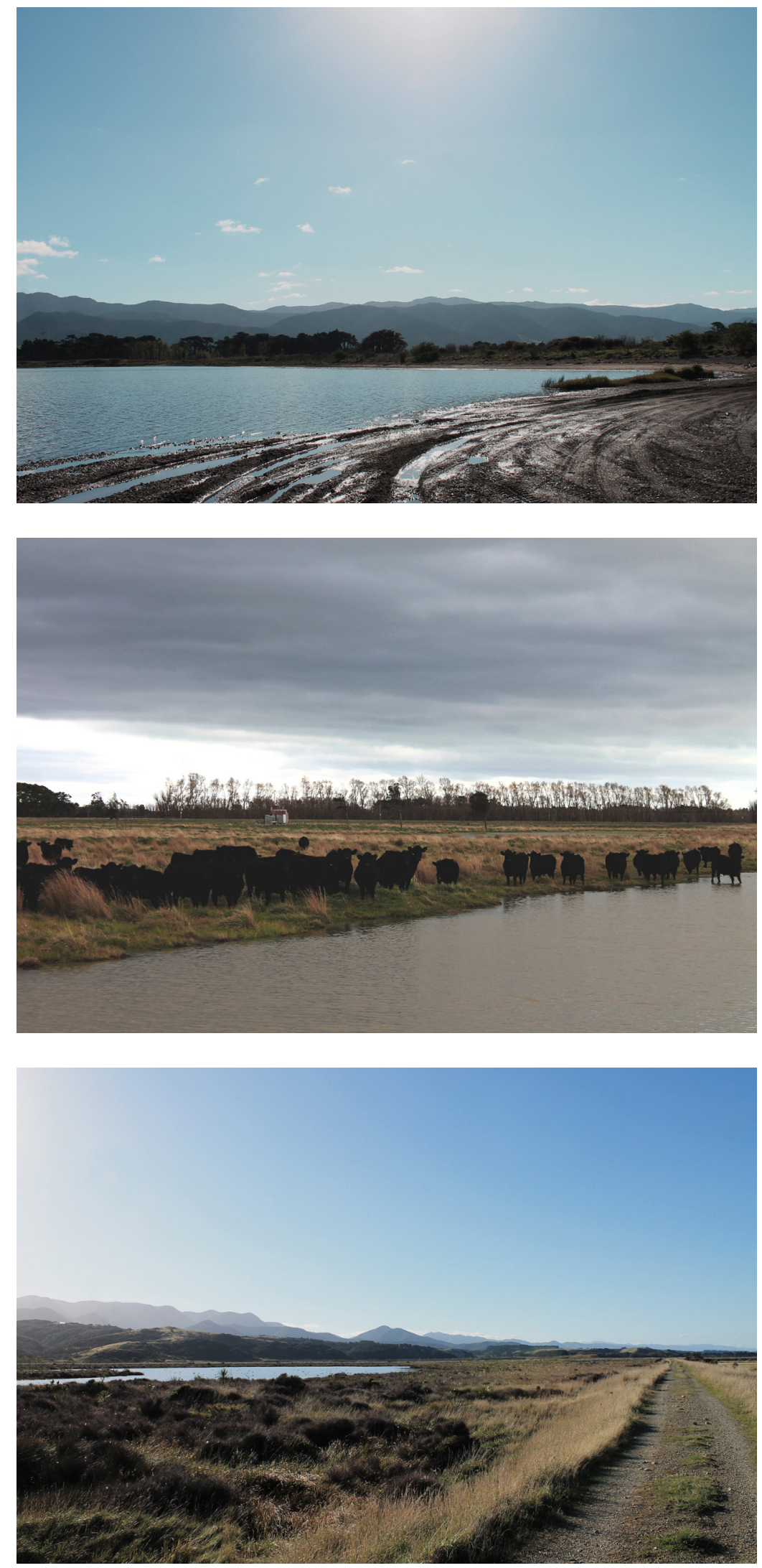

Fig 2.0 Lake Domain

Wairarapa Moana Wetlands Park, Site 1.

Fig 3.0 Wairio Block

Wairarapa Moana

Wetlands Park, Site 3.

Fig 4.0 Pounui Lagoon

Wairarapa Moana

Wetlands Park, Site 5. 


\subsection{THE WAIRARAPA SCOPE}

South Wairarapa is situated in the Wellington region, located $62 \mathrm{kms}$ North East of Wellington City where land is becoming scarce. (Fig 2.0 - 3.0) South Wairarapa has a low population of 9,528 people and expanses of open space(Statistics New Zealand). Included in this open space is a unique lake system that has been artificially altered from the once flourishing natural wetland system, into a degraded and highly polluted water system. This degradation is typical of many lake systems within New Zealand. Lake Wairarapa has the interest of the Regional council and local iwi to improve the water quality, but limited financial assistance is available.

This thesis argues that existing public spaces can be used to improve water quality at a lower cost than traditional infrastructure heavy methods. Overall aiming to reduce the effects of surrounding development, improve water quality and raise awareness for the value of the landscape. Lake Wairarapa's hydrological system covers a large area of land, the opportunity for large scale design that effects both the larger scale ecosystem and the small scale human activity is already evident at this site. Once dominated by wetlands, the expansive flat landscape only requires minor intervention, through wetland restoration in key areas to have a positive impact on the larger ecosystem.

The combination of large and small scales interventions could revitalise existing public space, harnessing natural function of the unique ecosystem that once dominated the land. 


\subsection{WAIRARAPA MOANA IN CONTEXT}

Wairarapa Moana is defined by this research as "lakes Wairarapa, Oneke and the rivers, streams, wetlands, lagoons and adjacent land that are all part of the same interconnected ecosystem." (Te Whaiti, Rhodes and Barnett) Surrounded by expansive amounts of land owned by the Greater Wellington regional Council (GRWC), the Department of Conservation (DOC) and the South Wairarapa District Council (SWDC) and containing one of the largest wetland systems in the Southern North Island, this sensitive water system is in serious decay. Wairarapa Moana as a research site has the potential to support landscape design that mitigates the effects of the intensive dairy farming, creates cultural identity for the local people of the land, and invites human interaction through the careful design of public space.

Artificial flood and water table alterations have transformed the landscape. Formerly a wetland and lagoon system, now pastoral land for dairy farming. Although the feasibility of removing the dairy farming and returning the landscape to wetlands is unrealistic, mitigating the effects of this practice through the readily available publicly owned land has much potential. The weak relationship between humans and the land has resulted in poor water quality and silt build-up within the lake.

To achieve landscape resilience, the application of respect for the environment is first needed. Considering its natural function, and taking from the land only what is absolutely necessary are two key principles that are needed. Outcomes driven by these principles will signify the end of decay for the lake system and the begining of water quality improvements. Protecting the natural ecosystems for the future.

How can a respectful view of nature be used in landscape architecture to achieve resilient mitigatory design that benefits the people of Wairarapa and their natural environment? 


\subsection{LITERATURE REVIEW}

The value of the natural landscape that supports the world we live in is continually changing. Once highly respected and valued for the natural resource and amenity value it possessed, it currently stands as an afterthought of development. A shift in thinking is again beginning to dawn, (Fig 5.0) starting with landscape architecture theorists such as Prof. Kathryn Moore argues that the landscape needs to be treated as a base layer for design, not an afterthought (Moore).

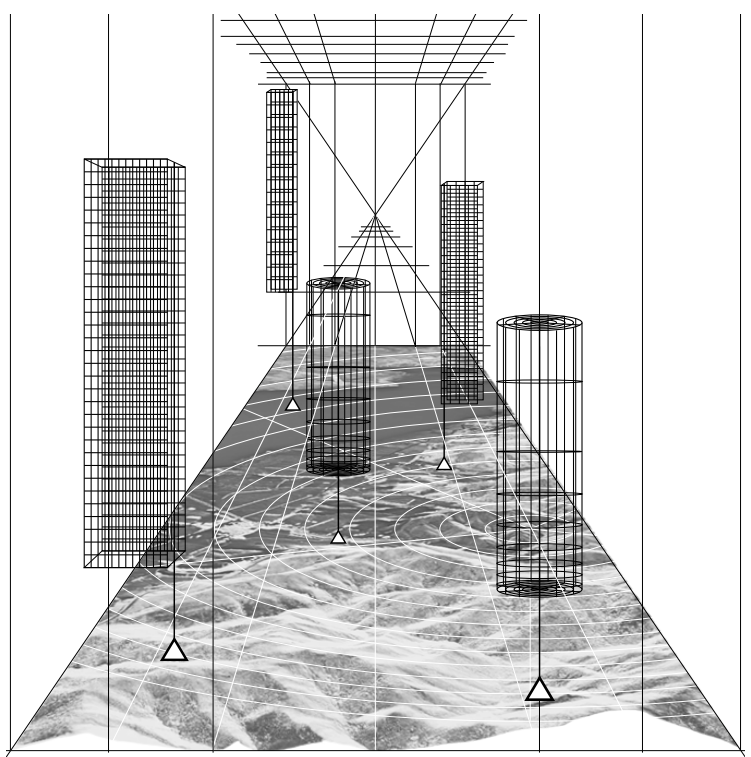

Fig 5.0 Landscape Perception Diagram

\subsubsection{LANDSCAPE FROM THE BASE}

Rather than being a decorative postdesign addition, landscape needs to be considered as the fundamental beginning. The power it holds within people and its ability to shape the world we live in subconsciously means it is a crucial consideration. The concept of eidetic is termed by Corner to help define the power a space has additional to environmental and experiential qualities.

"Landscape reshapes the world not only because of its physical and experiential characteristics but also because of its eidetic content..." (Corner 1)

By first considering landscape, development has the potential to work in synchronisation with the natural environment saving the land from a detrimental effect. A much more holistic outcome can be achieved between infrastructure and the natural environment if design ensures these two aspects work together, changing the 
current thinking of what infrastucture is and how it functions. However the power to positively inform people's subconscious participation in life can simultaneously be achieved on another level, through the way we design our environment. Although subjective, the power landscape has to inform our environmental milieu is invaluable. Yet still little protection is granted, protection that easily faltered.

Considering key landscape concepts for all design is crucial in the modern world, however like all drivers, some control is required. Alex Wall suggests in his essay 'Programming the Urban Surface' that landscape urbanism should cover every aspect of the land we inhabit. In Wall's opinion cities should spread and merge with the surrounding space to creative an 'interactive surface' that allows the landscapes natural self-organisational traits to dominate.

"The grafting of new instruments and equipment onto strategically staged surfaces allows for a transformation of the ground-plane into a living, connective tissue between increasingly disparate fragments and unforeseen programs." (Wall)

By allowing urbanism to sprawl uncontrolled into all urban spaces, bringing with it the self-organised surface, the natural landscape forfeits some of its own power.

This would result in the creation of a monotonous surface that removes the unique diversity that landscapes possess, their character, cultural identity and to some degree amenity value. The only aspect of the landscape that is retained is the functionality of specific programmes. The ability of a landscape to allow humans to escape the urban milieu would be stripped under Wall's theory. Allowing urban and natural spaces to coexist is the future of design, however limitations on this theory are needed. Ensuring the natural landscape is preserved is imperative, especially in New Zealand where the cultural identity of the landscape positively informs 
society through a deep connection with the land. The balance between urbanism and natural landscapes are sought in this thesis through a designed environment. This environment however needs to consider a multitude of scales to reach the objective of improving water quality of the Wairarapa Moana ecosystem.

\subsubsection{ENVIRONMENTAL INFRASTRUCTURE}

Infrastructure is a necessity of every space that supports human inhabitants. Landscape infrastructure is equally as important yet requires careful consideration when altering natural systems. Ecology and infrastructure form a vital relationship that is currently missing in New Zealand's landscape. Common practice sees heavily artificial infrastructure forced into the land, driven by functionality. There is a negligence of respect given to the complexity of natural ecological systems. Little understanding results in the destruction and harmful manipulation of our natural environment.

Using the natural system of a site to undergo tasks, which traditionally infrastructure would be introduced to do, seems to be a logical yet underutilised solution. Existing predominately in theory, this inclusion of the environment into infrastructure design is the core principle to be tested through the practical application of water remediation in this thesis. 'Wetland Design' by Robert L France explains how the natural environment is being destroyed by development, 
with little to no attempt at minimising the adverse effects this has. France proposes a new way of designing infrastructure that sees the landscape being considered and more importantly used to its advantage for its water treatment values. This achieves the same outcome that traditional rigid infrastructure would typically achieve, while having a positive influence on the environment and larger ecosystem.

The concept behind a city having green infrastructure within our natural environment stretches back into the history of landscape architecture and urban planning. Sir Ebenezer Howard describes an interesting relationship between humans and the natural environment in his 1965 book 'Garden Cities of To-morrow'. Howard argues that that cities need to be planned with large spaces for garden and productive agricultural land, all with a close enough proximity that people can easily access them from any dwelling within the city. Development of Howards' garden city theory brings the three key aspects of gardens, agricultural land and urban areas into one, arguably key elements of landscape resilience. Although Howard's concept of a utopian city can be dismissed, the relationship between urban areas and green space is still current. Benedict and McMahon state in their book 'Green Infrastructure: linking landscapes and communities', that the first integrated 'green infrastructure' was designed in the 1990's in the United States, this infrastructure is however very basic supporting corridors of green space over a large urban area. Little development in this field of research creates an opportunity to explore environmental infrastructure, a development of green infrastructure that sees the function of the environment taking lead over artificial function.

This design research is bringing on board and testing principles of functionality within 'green infrastructure' which are inclusive of environmental considerations. As well, this study is researching on how a landscape can function as water infrastructure while holding cultural and amenity values. Supporting France's argument that landscape architecture can be used as a medium to create space that 
encompasses human, environmental and functional factors into one holistic design.

"A great opportunity now exists to reshape and positively advance... through the field of landscape architecture, whose design motivations encompass a more pluralistic view of the natural environment..." (France)

The ecological function of such designed landscapes is equally as important as the human experience, finding the balance is imperative in a successful design. Nina-Marie Lister addresses this issue in Large Parks. The the idea of a 'designer ecology' is argued to be used a lot in public park space on a similar scale to this research. This leads to ecological function being more of an aesthetic gesture rather than a key functional element. With open space being so precious in New Zealand, design must move away from 'designer ecologies' which are commonly seen through the country. Green washed landscapes that have no positive effect on the larger ecosystem in which they exist. Modern design needs to function ecologically, while allowing human interaction to simultaneously happen within the space to encourage an interplay between recreation, learning and cultural identity. A theory of landscape architecture that remains uncommon in New Zealand's expanses of designed spaces. 


\subsubsection{CULTURE IDENTIFIED}

Culture within landscape is very subjective, identifying cultural landscapes is highly depended on many factors that change with every site. A Place to Stand by Raewyn Peart argues that the naturalness of New Zealand is being put under threat by development, the protection of important landscapes is crucial but hard to prove with solid facts when development has such high finical feasibility. Cultural landscapes with natural heritage need protection because of their unique ability to positively inform social constructs.

"The purpose of protected landscapes reinforce the positive aspects of the relationship between people and the land" (Peart, 7).

In support of this argument that the natural landscape needs protection, the Maori culture can be explored. Specifically the value of respect that this culture holdswith the land. Infrastructure design typically has little respect for that land it consumes, instead functionality holds the core value, stripping the landscape of its natural character. To provide this protection our remaining natural spaces require design that ensures the essence of the landscape character is retained, strengthening our cultural identity.

Many concepts within the Maori culture can be applied to the landscape, these concepts can then be used alongside design principals to achieve outcomes that are respectful of the natural environment. The concept of Kaitiakitanga within the culture is expressed as guardianship of the land, people and the landscape are one unity with neither having more power over the other. The principle of respect for the natural environment, and providing protection for the future through guardianship, is an important Maori value that is embedded in this design research. Taking from the land only what is vital for survival is another value that is instilled in the Maori culture. Exploitation of the landscape as a resource is common practice all around the world. A stronger cultural identity and realtionship with the land can be achieved through the application of respect. 


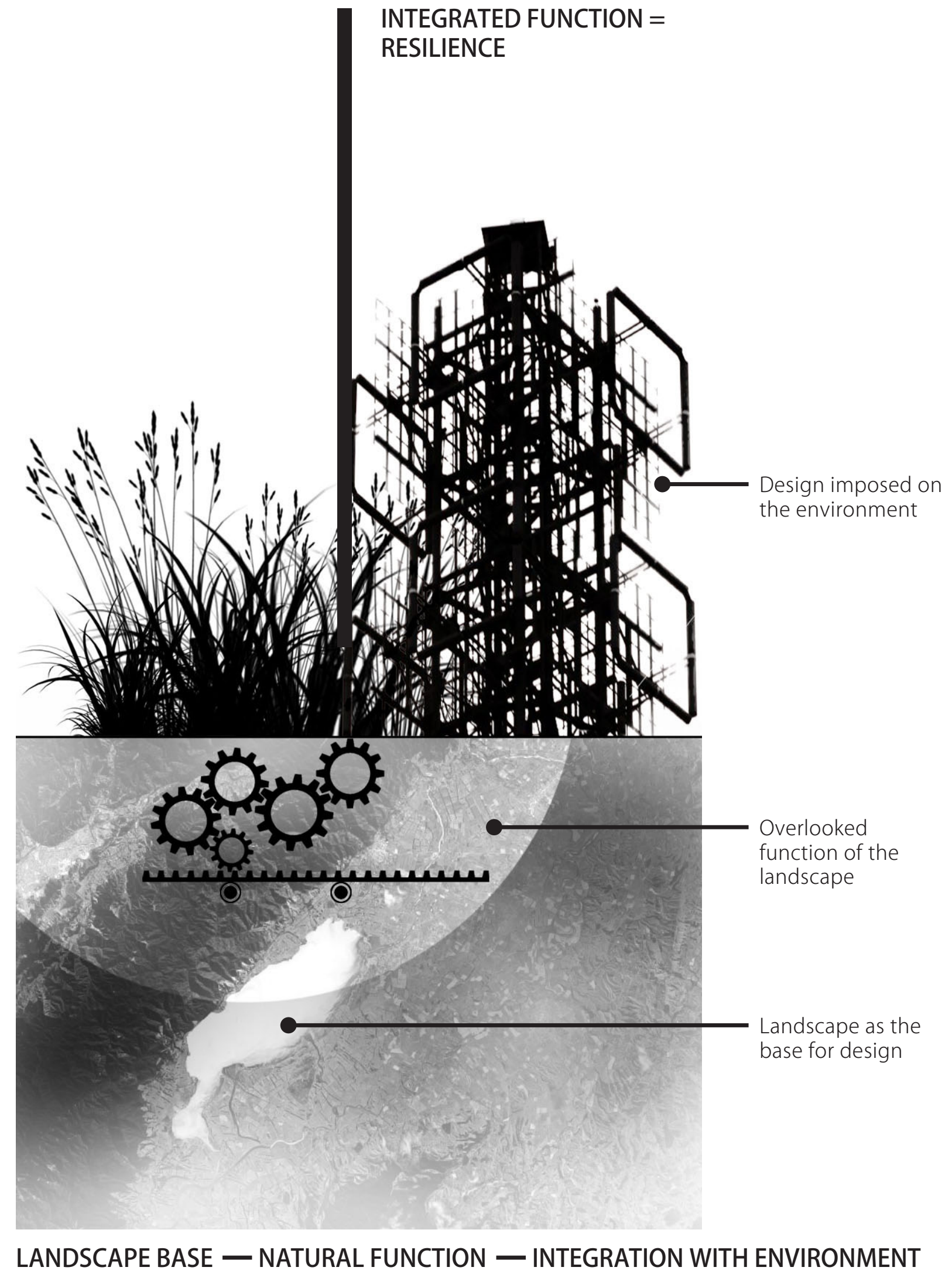


Research in the field of natural heritage in landscape architecture, cultural landscapes, is ever increasing. The linking of this research with ecology and infrastructure design is however not imminent, this thesis aims to explore the theoretical void through practical application. By researching initially how principals from Maori culture can be used to inform design, a landscape architecture methodology will be applied resulting in a design outcome that protects, and informs a positive relationship between people, and the land.

"The reappearance of landscape in the larger cultural imagination is due, in part, to the remarkable rise of environmentalism and a global ecological awareness" (Corner, Terra Fluxus)

Culture in landscape is now identified as a crucial element, ensuring its protection is imperative. Corner states in his article 'Terra Fluxus' that the new found interest in landscapes and their protection as an integral aspect of culture, is partially due to the interest in environmentalism and ecological awareness across the globe. The newly discovered interest in the fusion of landscape and culture is something that can be researched further to create design that offers more quantifiable additions to the world's cultural identity. In particular this theory has potential to be explored in New Zealand where the landscape already forms such a large part of the native culture. This thesis follows this momentum to develop landscape design that positively informs New Zealand's cultural identity.

\section{Fig 6.0 Literature review diagram (Opposite)}

Summarising diagram of key concepts gained from literature review. 


\section{APPROACH}




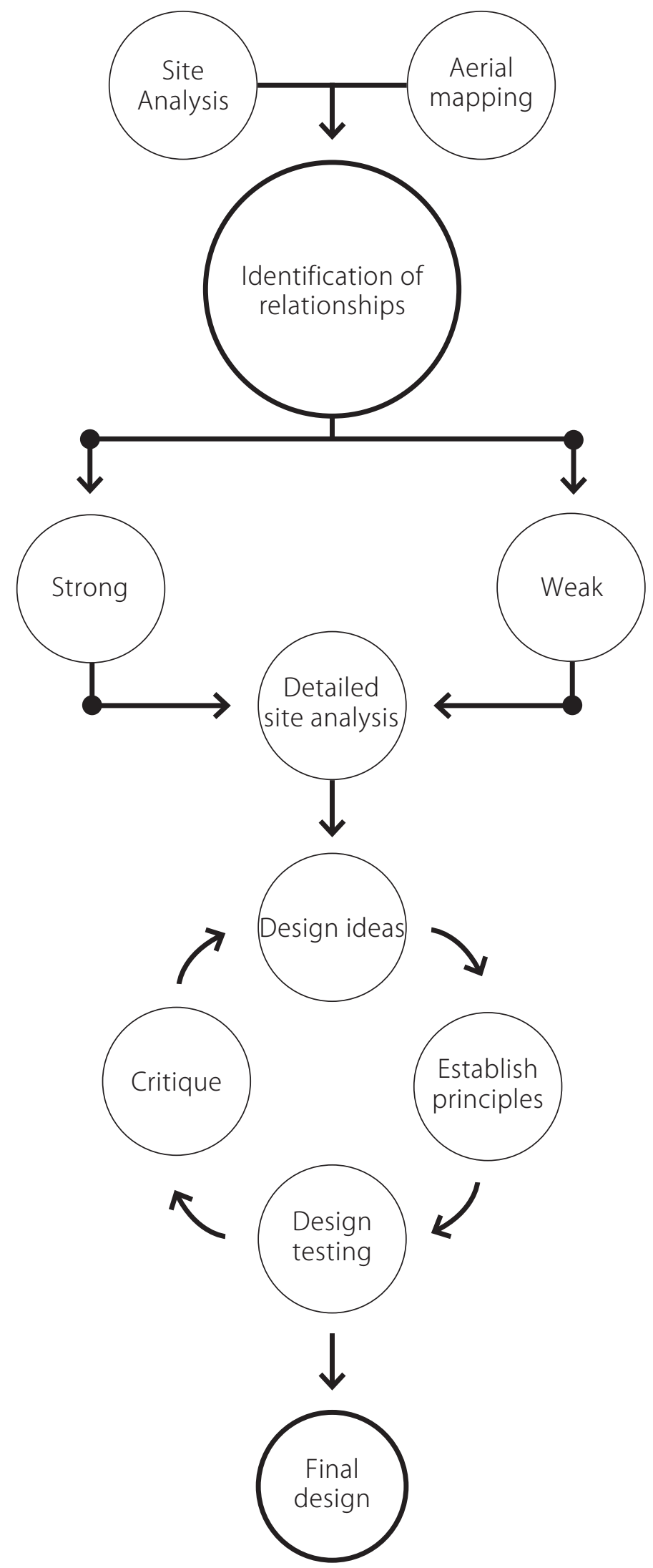




\subsection{METHODOLOGY}

This thesis explores how landscape architecture design can have a positive impact on public land, integrating water infrastructure into the region. Focusing on the issue of water quality in the South Wairarapa Region and using generative design as a vehicle to improve people's relationship with their land. Exploring how publicly owned land could be used as a multifunctional space that is respectful of the surrounding natural ecosystem, creates cultural identity and begins to mitigate the adverse effects of development.

Carl Troll, a very influential geographer in the $20^{\text {th }}$ century was one of the first to conduct landscape analysis through aerial photography, used to identify relationships between vegetation and environment. This thesis follows Troll's method of analysis to identify were weak and strong relationships exist based on vegetation; native vegetation showing a strong relationship and exotic vegetation showing a weak realtionship. The identification of these relationships will generate a foundation from which a rigorous site analysis explores the landscape in more specific detail.
A regional scale analysis explores and identifies the larger opportunities in the South Wairarapa. Following the regional scale a site specific analysis highlights the opportunities of the selected sites at an intimate scale. One site that is representative of the entire selection will be used to form principles and test design iterations. A cycle design method here allows principles of design and design iterations to continualy evolve as the thesis progresses. To ensure this research is continualy generating new design outcomes, iterations will be assessed against principles of design that are established in the literature review and site analysis phases. (Fig 7.0)
Fig 7.0 Methodology process diagram

(Opposite) 


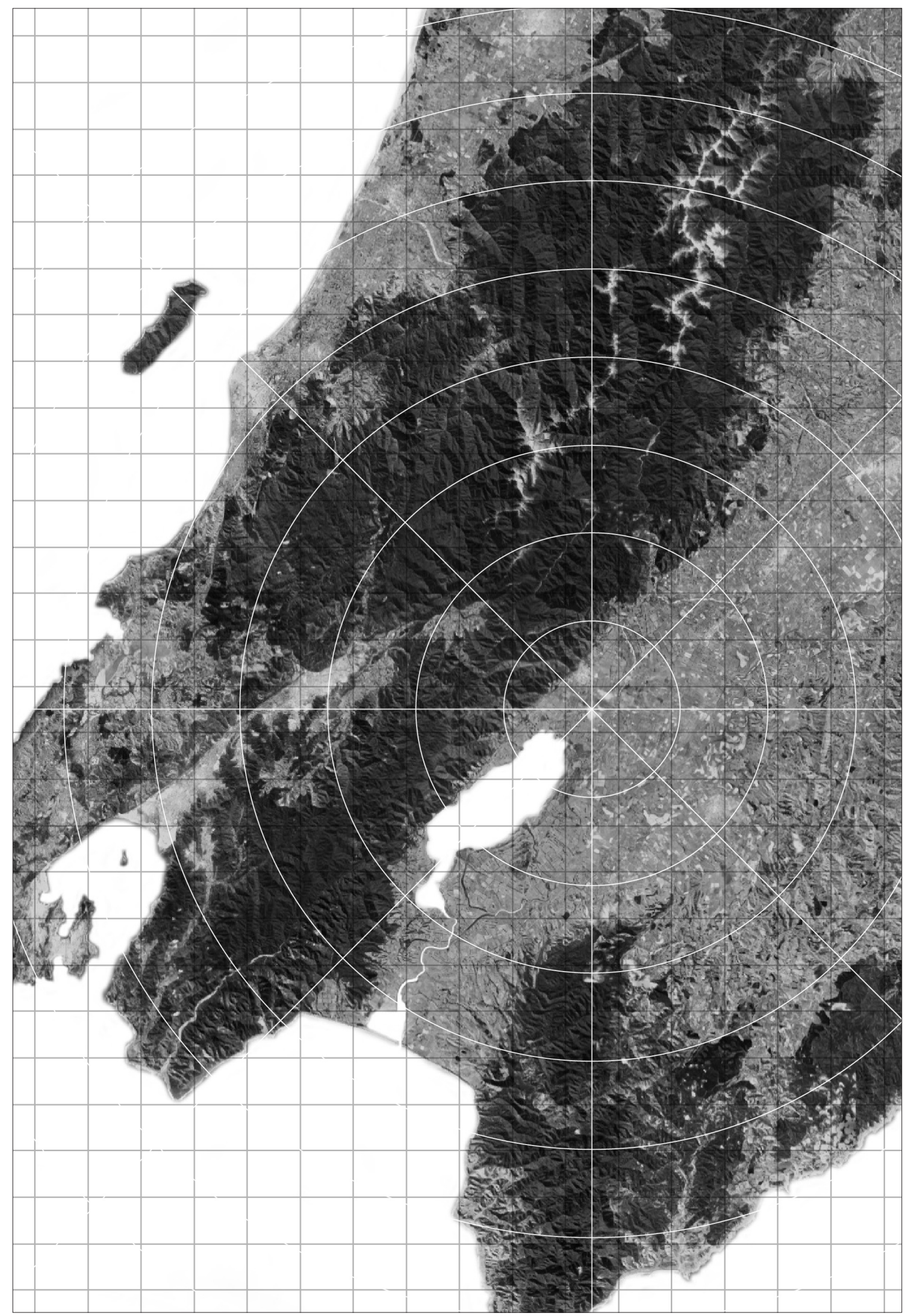




\subsection{SITE ANALYSIS}

The South Wairarapa region contains a unique natural landscape and ecosystem that is littered with human intervention and rigid infrastructure. Locating poor land use will enable the mitigation of the adverse effects detrimental infrastructure has on the natural landscape. The hydrology of the region begins to define the limits of potential intervention and what sites are most suitable to support landscape architecture design. The exploration of vegetation provides further justification for site selection, highlighting the amount of native flora in the region. Finally the land governance of the South Wairarapa ensures that this thesis is able to focus on achieving mitagatory landscape architecture design without additional political issues. Each of these requirements for design are evident in the South Wairarapa region.Through a rigorous analysis the specific sites for intervention will be identified and developed through the design phase.

\subsubsection{SOUTH WAIRARAPA SITUATION}

Wairarapa Moana, situated in the South Wairarapa Region contains a sensitive lake ecosystem that has undergone many transformations throughout its history. Lake Wairarapa, a shallow coastal lake, currently has such poor water quality that it is in a supertrophic state (Perrie and Milne). Recreational use of this lake is not recommended as the water quality can cause illness. Intensive dairy farming in the lakes catchment is partially responsible for pollution, alongside sewage and industrial discharges into the hydrological system. The lake however still provides habitat for many threatened species of native flora and fauna, giving Wairarapa Moana national significance for ecological value. (Fig 9.0)

Fig 8.0 South Wairarapa context (Opposite)

Fig 9.0 Wairarapa Moana infographic

(Following) 


\section{NITROGEN | SUPERTROPHIC STATE SACCHI DEPTH PHOSPHORUS CHLOROPHYLL \\ Lake Wairarapa has degraded water quality, characterised by elevated concentrations of nutrients and algal biomass and poor water clarity.}

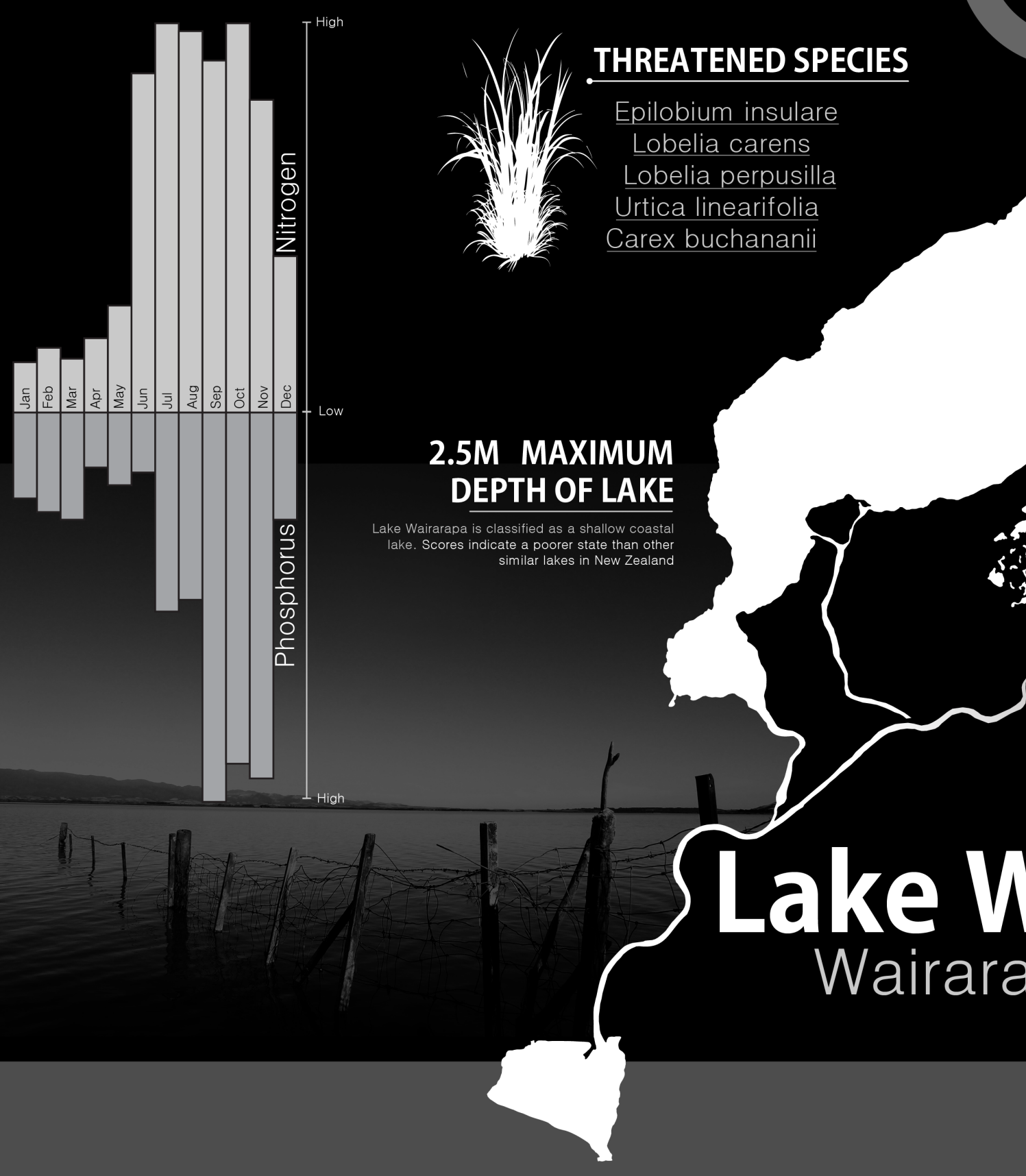


Hazardous activities from 384 registered sites in the Wairarapa Area

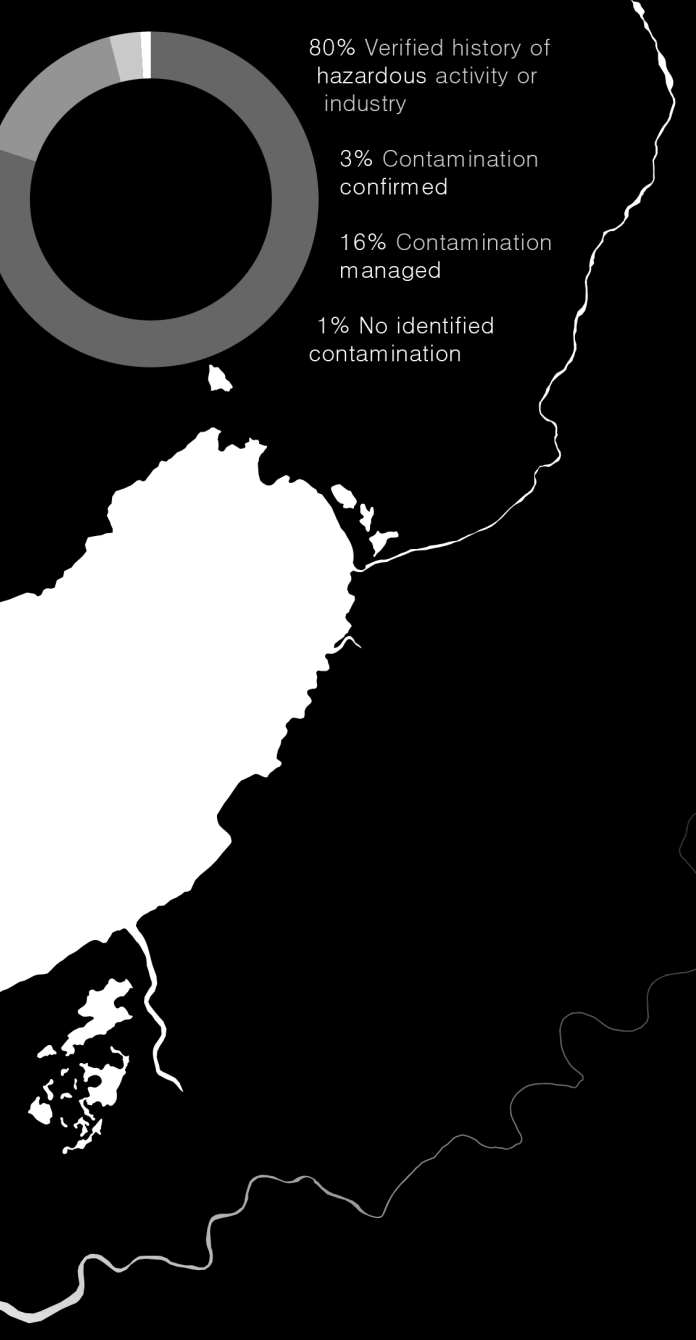

Providing Habitat

\section{BIRD SPECIES}

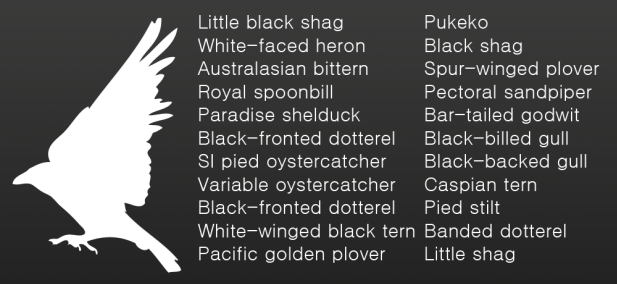

Annual average data vales, Lake Wairarapa 200
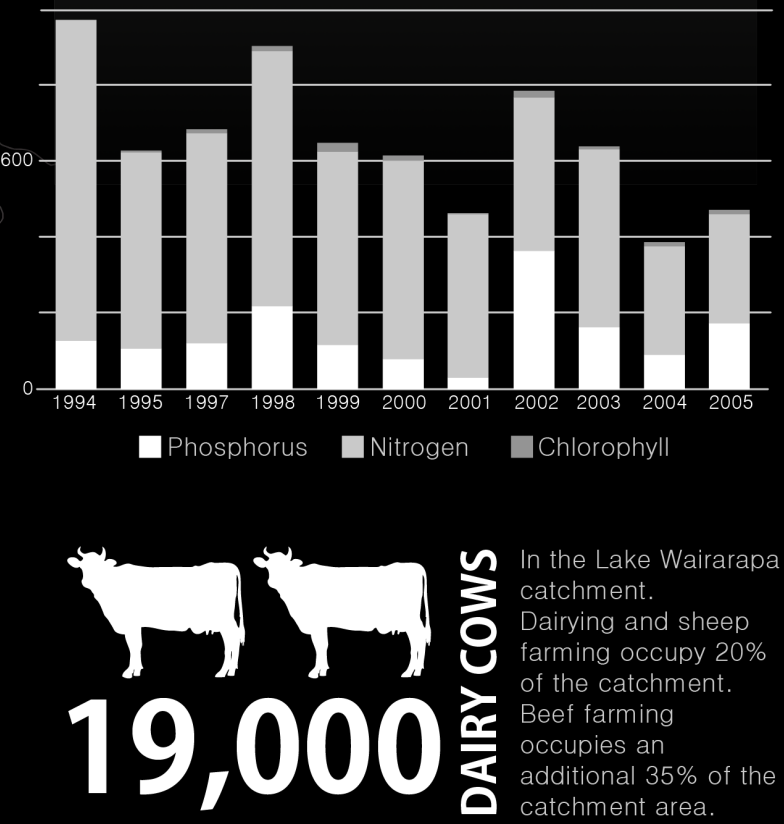

Wairarapa irapa moana 


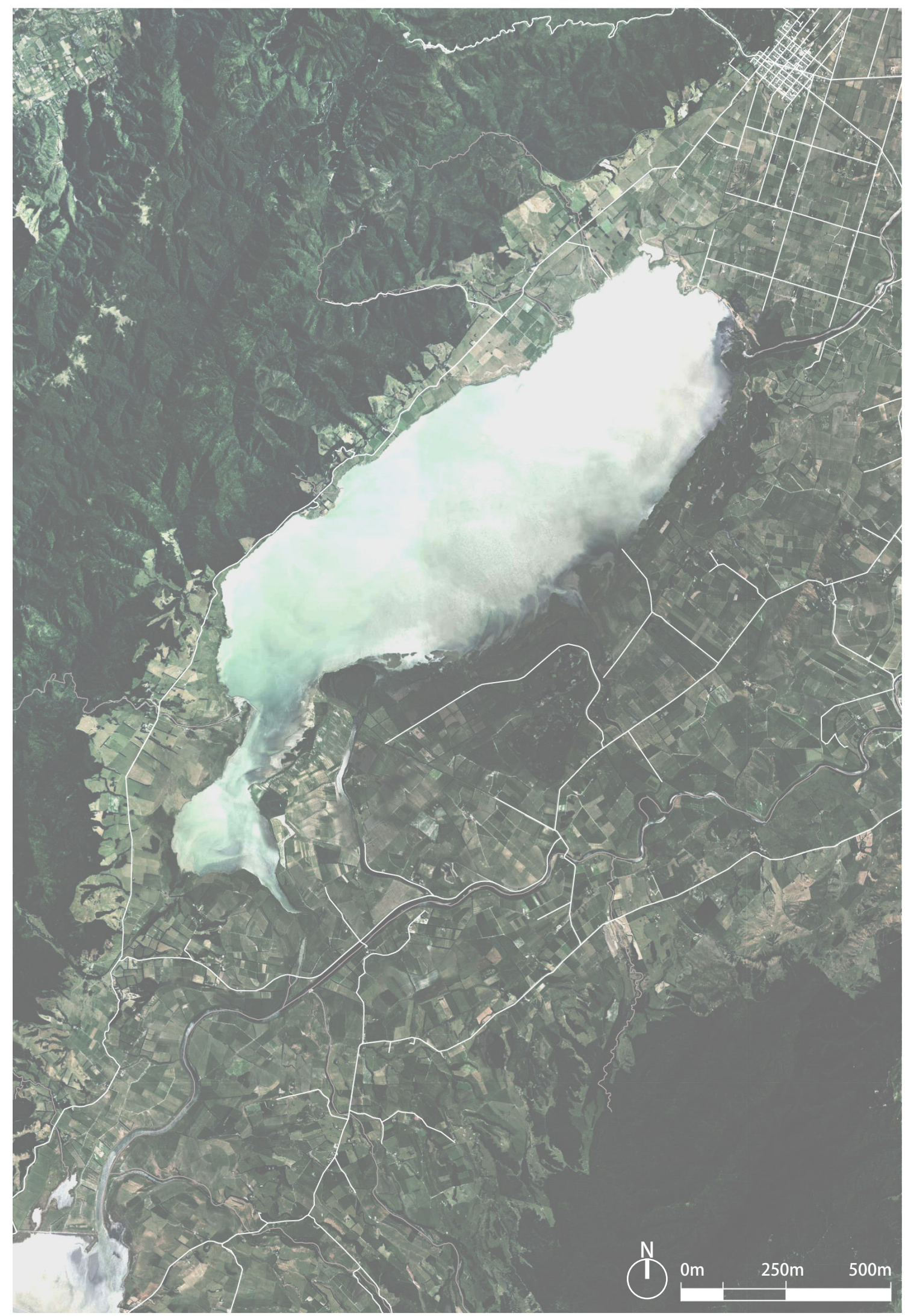




\subsubsection{BUILT INFRASTRUCTURE}

The South Wairarapa region contains many different types of traditional built infrastructure that was historically necessary for human settlement, most of this infrastructure has come at a cost to the natural environment. A major form of infrastructure are roads, providing important access to the entire region. Road infrastructure creates circulation and unity.

Roads within the South Wairarapa have a close relationship with the waterways, typically located very close the water runoff containing various pollutants from vehicular traffic which enters the hydrological system. Although the removal of road infrastructure would have a positive effect on the natural environment, it would impact the accessibility of the region. This gives an opportunity for design to explore how the pollution to the hydrological system can be mitigated or improved through landscape architecture.
Map key:

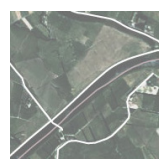

Road Infrastructure providing vehicular access to Wairarapa Moana. 


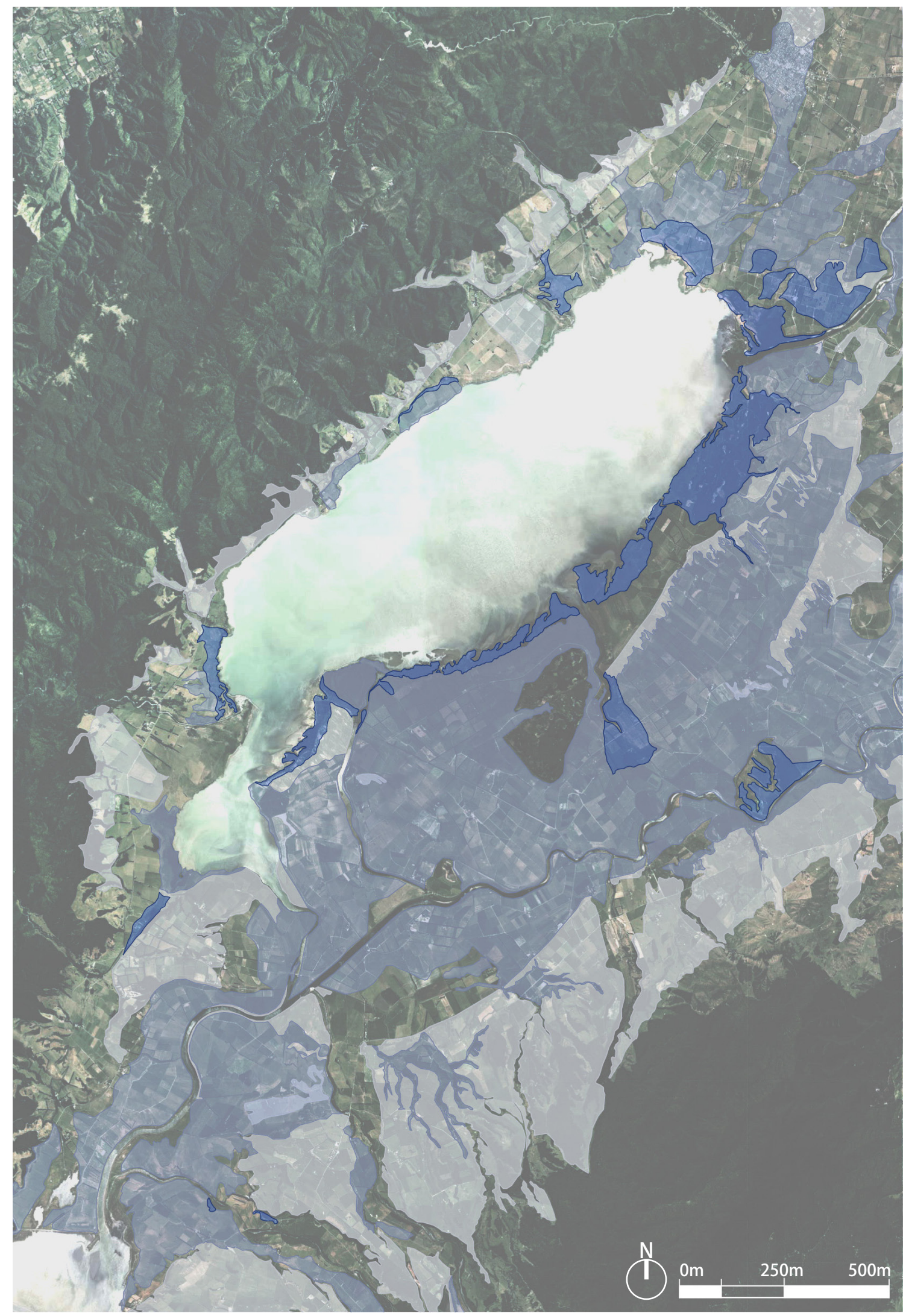




\subsubsection{HYDROLOGY}

Artificial management of the South Wairarapa hydrological system has severely altered the function of the natural waterways of the region. Soil moisture levels indicate types of flora that are best suited to specific areas. The South Wairarapa was once a wetland system therefore the soil moisture levels remain high.

By identifying the level of ground water through soil moisture data, the best outcome for the land can be achieved. Hydrological systems are very sensitive and expansive, little alteration can have a large effect on the ecosystem. Any further design of this area will need to accommodate the natural environment. The lake water levels are currently artificially altered and the direct result is raised ground water levels. Another more detrimental effect is the restriction of pollution-ridden sedimentation to be flushed from the lakes hydrological system. This study integrates the increased soil moisture and how that can be used alongside design to reduce the harmful effects on the hydrological system.
Map key:

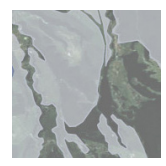

Moderate Soil moisture levels.

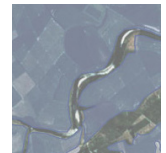

High soil moisture levels.

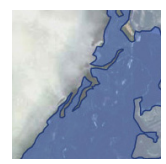

Very high soil moisture levels.

Fig 11.0 Regional hydrological analysis

(Opposite) 


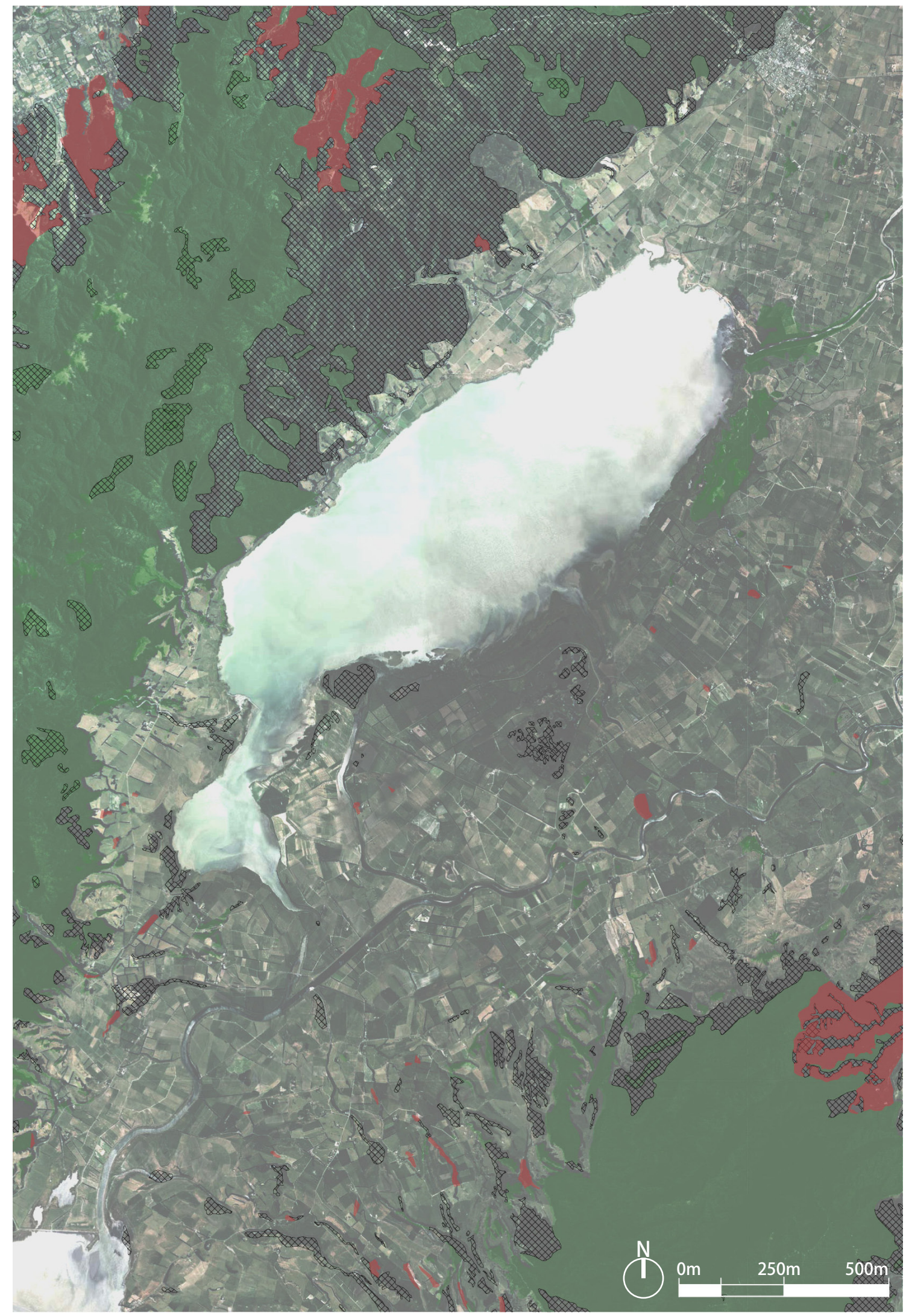




\subsubsection{VEGETATION}

The identification of how human settlement has had an impact on the natural environment can be identified through Troll's method of aerial analysis of vegetation cover, as shown in (Fig. 12.0) The function of a natural ecosystem relies on native vegetation. Exotic vegetation visible at a regional scale identifies a weak relationship between the people and their natural environment. The South Wairarapa vegetation cover identifies where the natural systems still exist, aiding in the establishment of sites for this research.

Exotic vegetation would need to be removed in order to align this research with principles of Maori's respect for the land. As little exotic vegetation is evident in the South Wairarapa region, there are few areas that are unsuitable for this study to take place. As this thesis research relies heavily on the function of the natural systems to inform design and improve water quality, it is imperative that this system is evident. Site specific analysis will clarify further any smaller sections of exotic vegetation, however the regional analysis shows little impact on the natural ecosystem.
Map key:

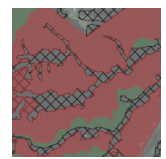

Exotic vegetation.

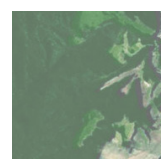

Native vegetation cover.

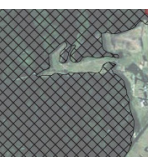

Scrub vegetation cover.

Fig 12.0 Regional vegetation analysis

(Opposite) 


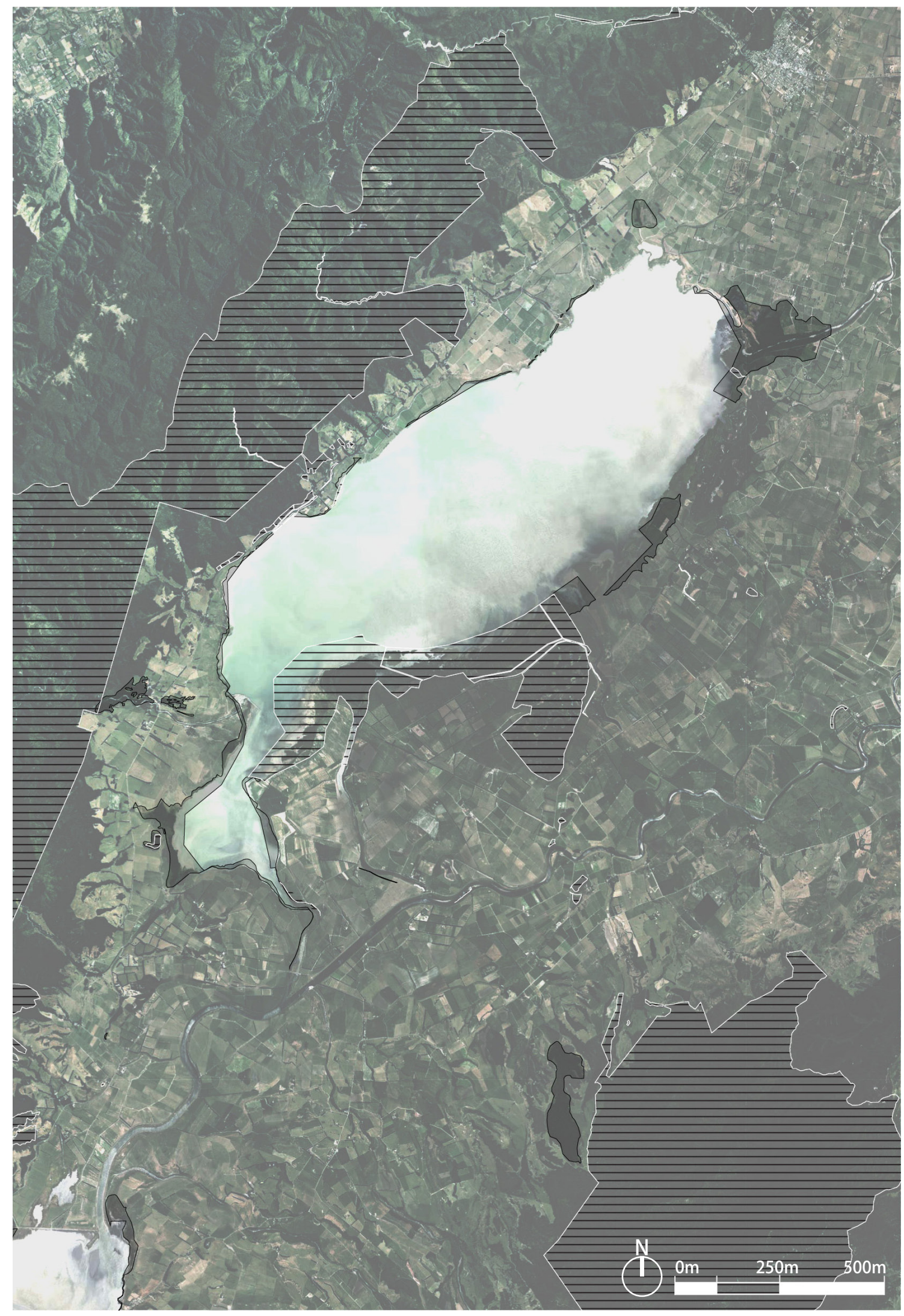




\subsubsection{LANDSCAPE GOVERNANCE}

The South Wairarapa region has a combination of private and publicly owned and managed land that is typical of New Zealand. The natural character of the land that still remains, even in its dilapidated state, is of interest to the Department of Conservation (DOC) alongside the Greater Wellington Regional Council (GWRC). Both of these stakeholders have an interest in the protection and enhancement of the natural landscape for the future. This thesis relies on existing publicly owned and/or managed land to allow for the focus of landscape architecture design to develop with more rigor. Excluding a political issue of land governance that could hinder design.

There is a vast amount of public land within the region, most of which is under utilised and has little environmental value in its current state. This provides an opportunity for design exploration of the land, leading to more productive and active spaces that align with the interests of DOC and GWRC Identifying which land parcels are owned and/ or managed by DOC will aid in the identification of specific sites for further analysis. Alongside current land, DOC. also have areas of land that they recommend to be protected for the future as they have significant natural value, further potential sites of interest

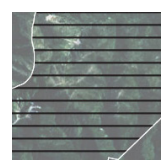

Publicly owned/managed land.

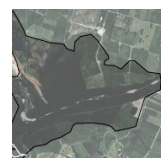

Recommended areas for future protection.

Fig 13.0 Regional public land governance (Opposite) 


\section{Wairarapa Moana Wetlands Park}

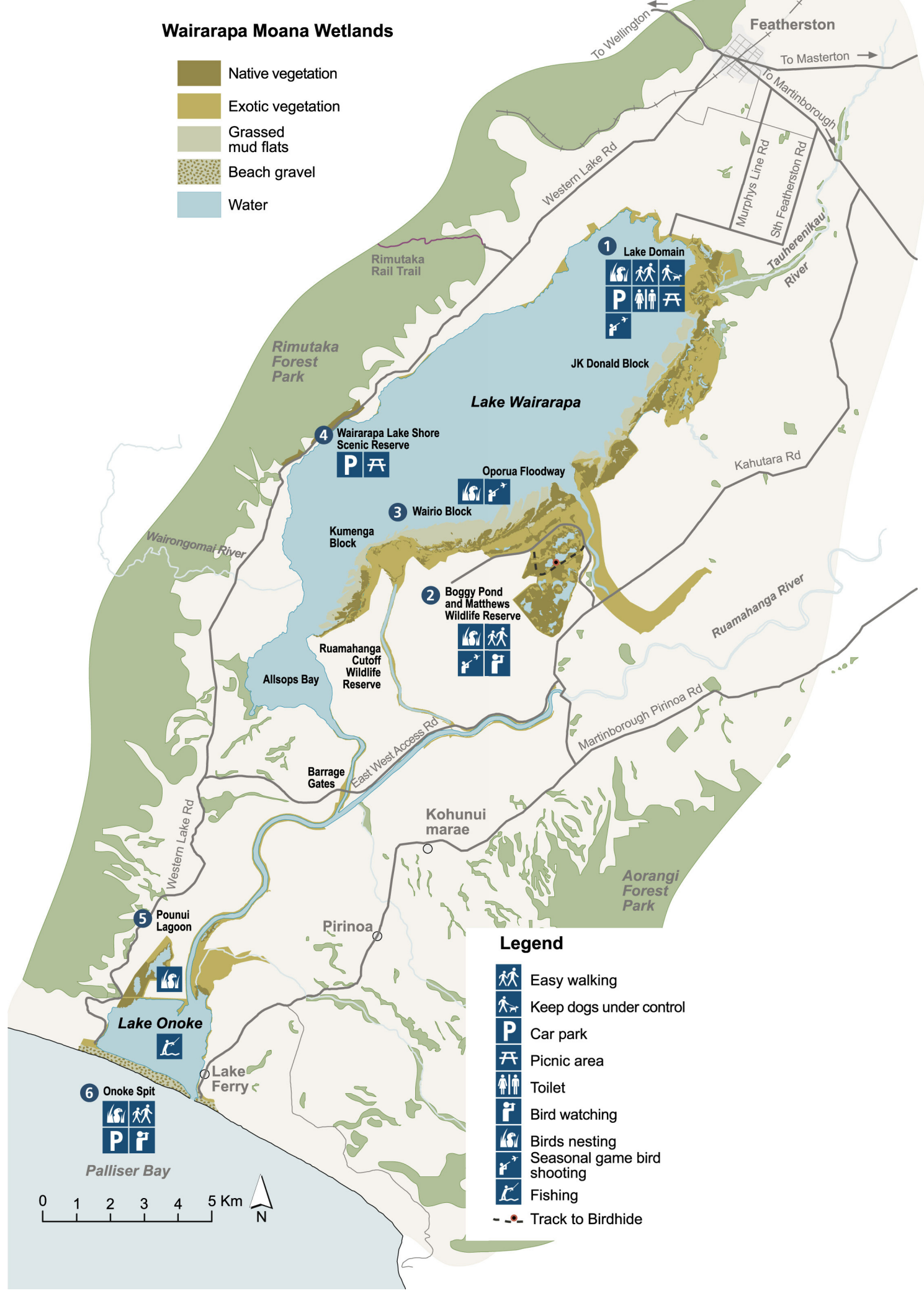




\subsubsection{WAIRARAPA MOANA}

Wairarapa Moana Wetlands Park is an area of land encompassing Lake Wairarapa, Lake Oneke and the publicly owned land that adjoins them. The GWRC has an interest in six key sites, with funding allocated to begin to revive this landscape with the intention of improving recreation in the area. To ensure the politics of land governance do not have a negative impact on the design progression of this thesis, these six sites are assessed against the research aims. Three key sites were selected as they have the required variables; publicly owned land, access to Lake Wairarapa, high soil moisture levels, access via roading infrastructure and containing natural vegetation.

Fig 14.0 Wairarapa Moana Wetlands park Map (Opposite)

http://www.gw.govt.nz/assets/Parks-andRecreation/Wairarapa-Moana/wairarapamoana-wetlands-park-brochure.pdf 


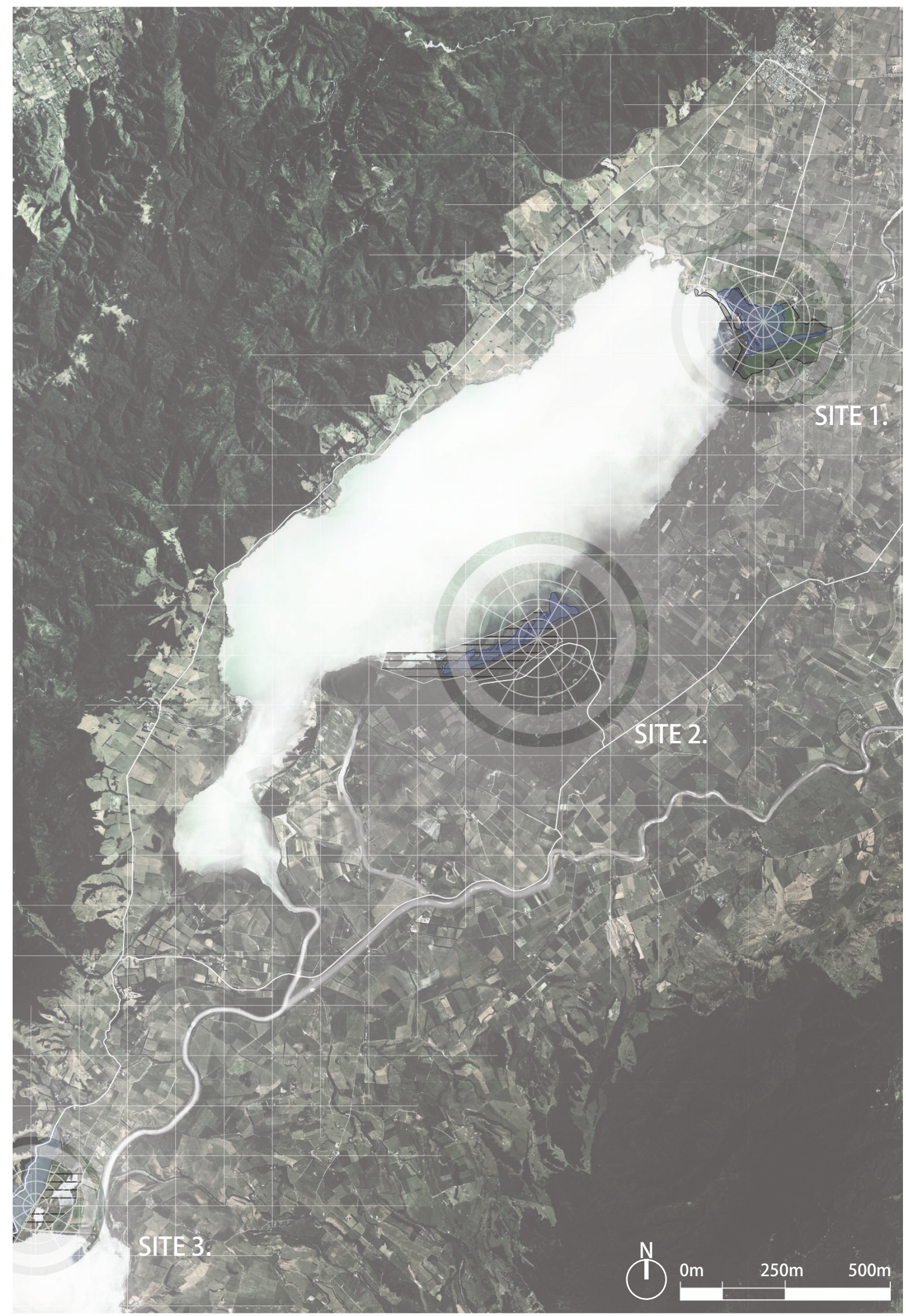




\subsubsection{CONCLUSIONS}

Undergoing a regional analysis, the South Wairarapa has many potential sites for this thesis to explore through design. The infrastructure in the region is minimal compared to that of other similar regions of New Zealand. This allows design to lessen the effects of this infrastructure while simultaneously improving the hydrological system of the area. Minimal amounts of invasive vegetation and exotic species also provide a perfect canvas for design to develop and harness the values of the native flora. These conclusions have been drawn from their respective identification through aerial analysis. The establishment of Wairarapa Moana, specifically the similar visions for the landscape, will ensure at all stages the feasibility of any design intervention is considered and remains practical. The removal of land governance issues reinforces the suitability of the South Wairarapa as a vessel for this thesis.

The conclusions of this analysis has led to the selection of three sites suitable for this research to explore in detail. The sites have been selected due to their aptness of present infrastructure, hydrological elements, vegetation cover and land governance. Each of these sites can be used to support environmental infrastructure that mitigates the adverse effects that development has had on this landscape, which will be explored in the following chapters.

Fig 15.0 Regional synthesised analysis

Analysis Map showing the conclusions drawn from the regional analysis including the three selected sites for research. 



\section{ANALYSIS}




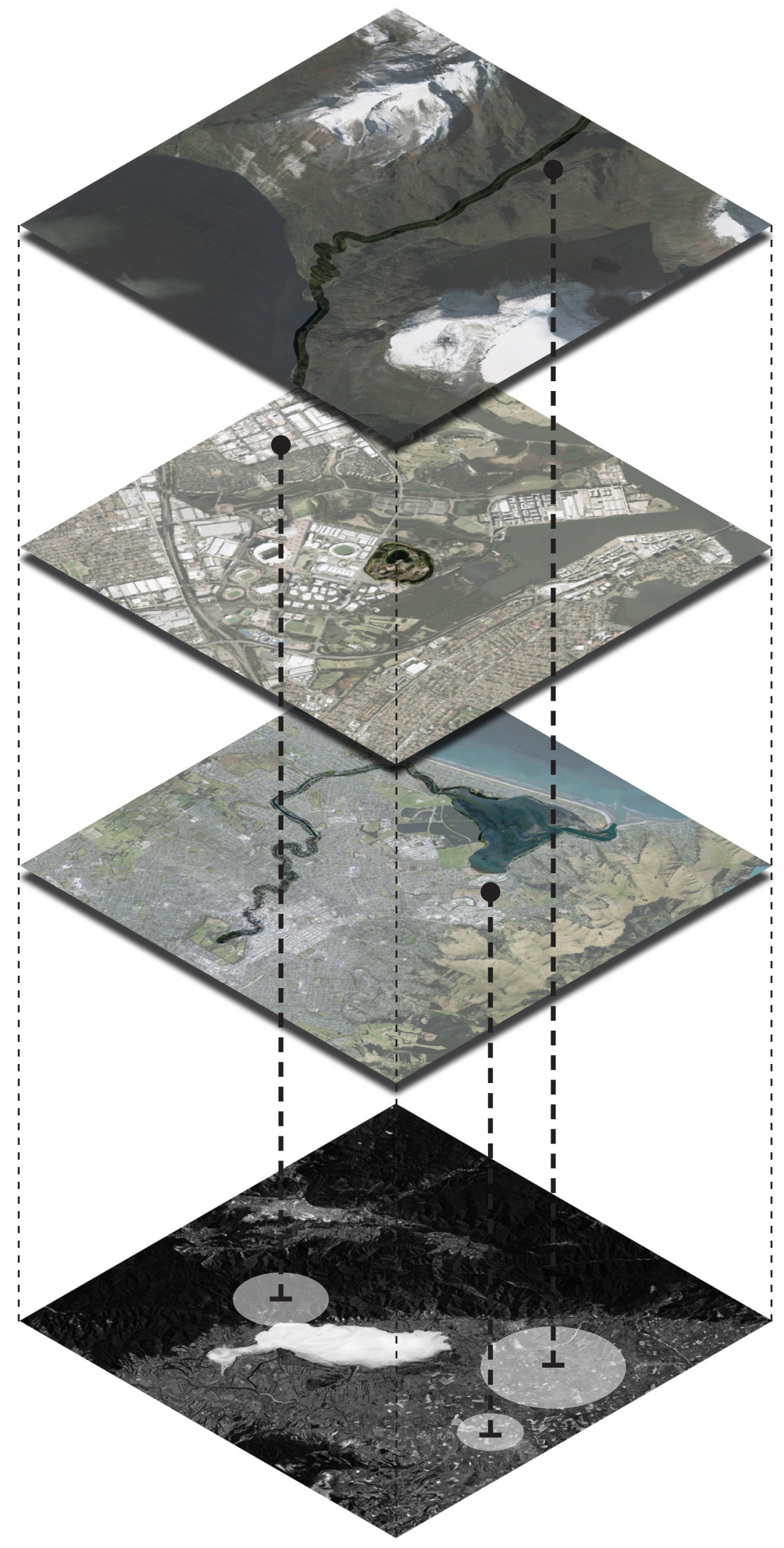




\subsection{PRECEDENT ANALYSIS}

Precedent analysis is used to explore existing disciplinary solutions of sites that hold similarities to South Wairarapa. Similarities such as scale, environment, protection values and infrastructure design. These existing solutions will then be applied to the site selected for this research to identify similar elements that can be adapted and developed to ensure the best outcome is achieved.

Looking at the design method behind the formal solution will allow new findings to surface and be transported to this study. Learning from the faults of these approaches will inform the development of resilient infrastructure in the South Wairarapa. (Fig 16.0)

Fig 16.0 Precedent analysis diagram (Opposite)

Taking the key ideas from each precedent and layering them on the selected site will encourage a generation of new ideas. 

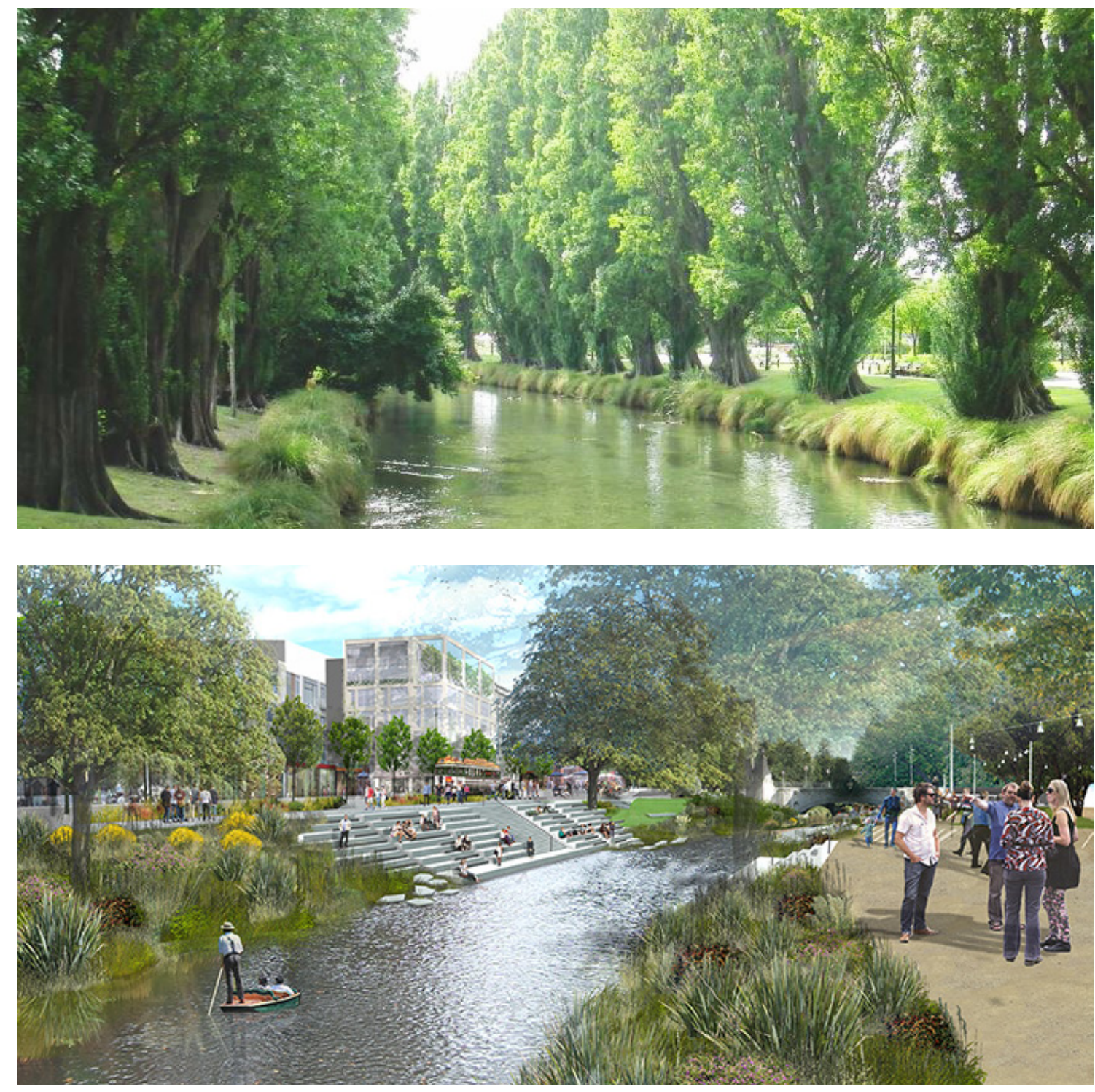

Fig 17.0 Avonhead River post construction (Top)

Fig 18.0 Avonhead River Render (Middle)

Fig 19.0 Aerial Image of Avonhead river (Bottom)

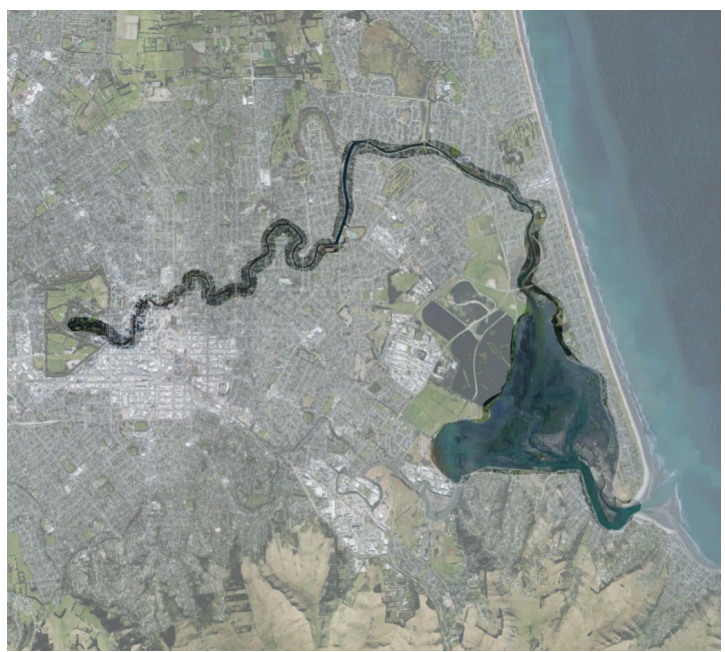




\subsubsection{AVONHEAD RIVER}

Situated in the heart of a city recovering from a natural disaster, the Avonhead River project is a refreshing synergy between ecology and landscape architecture design (Fig 19.0). Land that was once a natural wetland system and Native Kaihikatea forest now supports a large city. The Avonhead River flows through the CBD of Christchurch, New Zealand where it is leading the way in ecological design. Driven by the objective of enhancing natural habitat for native species of flora and fauna. One of the first projects of this nature, Avon River provides a built example of urban waterway restoration that is much needed in New Zealand. Ecologists and designers have come together to study the ecological diversity of this system, using their findings to improve the habitat for the future. (Fig 18.0) The river is broken up into three main typologies of design: Riffle Habitat, Run Habitat and Deposition areas. Each has an important ecological function, all three work together to achieve the overall goal of habitat restoration.

Respecting the history of the site, the exotic planting that currently exists on the banks of the river is retained and supplemented with new native planting (Fig 17.0). This will encourage more native bird life into the area. Sections of the river have been narrowed, which speeds up the flow of the water and ultimately increases the benefits. Silt build up on the river bed is flushed out, exposing the gravel beneath which provides an ideal spawning habit for trout. New riparian planting covers the banks of the narrowed sections, providing additional river edge habitat for eel and other aquatic species of fauna to thrive. Raingardens ensure any water runoff from the nearby road infrastructure is clean upon entering the river, previously heavily polluted water would enter the system with a detrimental effect on water quality. The banks of the river also boast a number of vibrant, multiuse public spaces allowing people to interact and learn about the sensitive ecology that they share the land with.

The combination of public space and environmental systems as drivers for design are increasingly being practiced. The integration of these two drivers to 


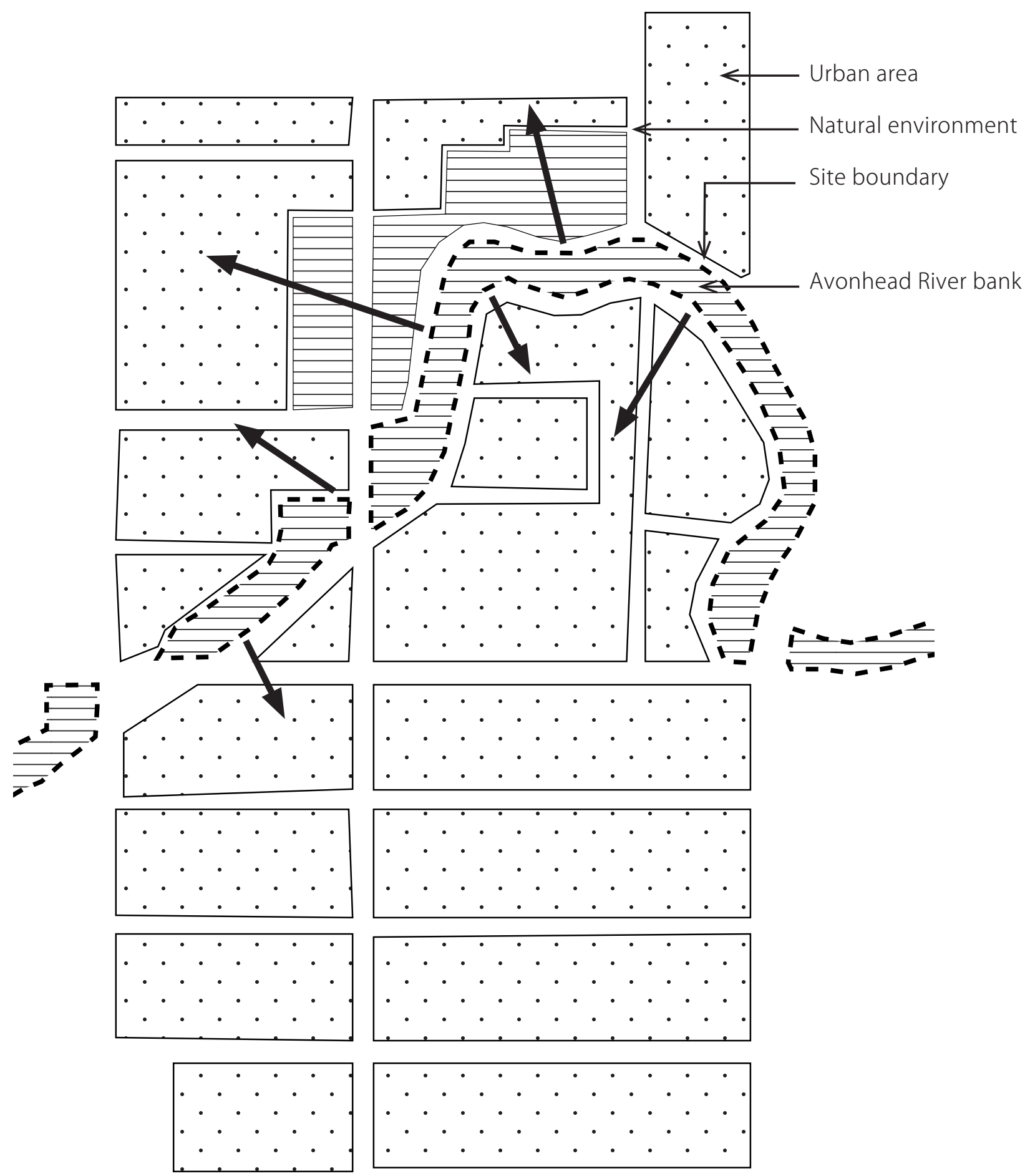


share the same space, and function as one unit, is still yet to be seen. Avonhead River successfully uses ecology to inform public space, however they still exist side by side as separate entities. Clear zoning between the river system and public spaces leaves an opportunity for design to push the limits of landscape architecture, combining these two zones to work as one, multi-functioning space.

The Avonhead River project is successful in creating designed urban spaces that are driven by ecology and providing restoration to the natural environment. Strategic areas of the river system have undergone significant change to create key spaces. The new journey allows people to learn about the natural habitat that is often taken for granted. Public space exists alongside the natural ecology of the site and the impact of human development on the river is mitigated. Awareness has been raised for the sensitivity of our natural systems and their need for future protection. An example of how landscape architecture can work with the natural ecology of the land to create a new typology of design
(Fig 20.0). One that has a positive effect on the natural environment, rather than a negative.

Fig 20.0 Avonhead River analysis diagram (Opposite)

Highlighted is how the intervention integrates within the urban context creating natural amenity in the city limits. 

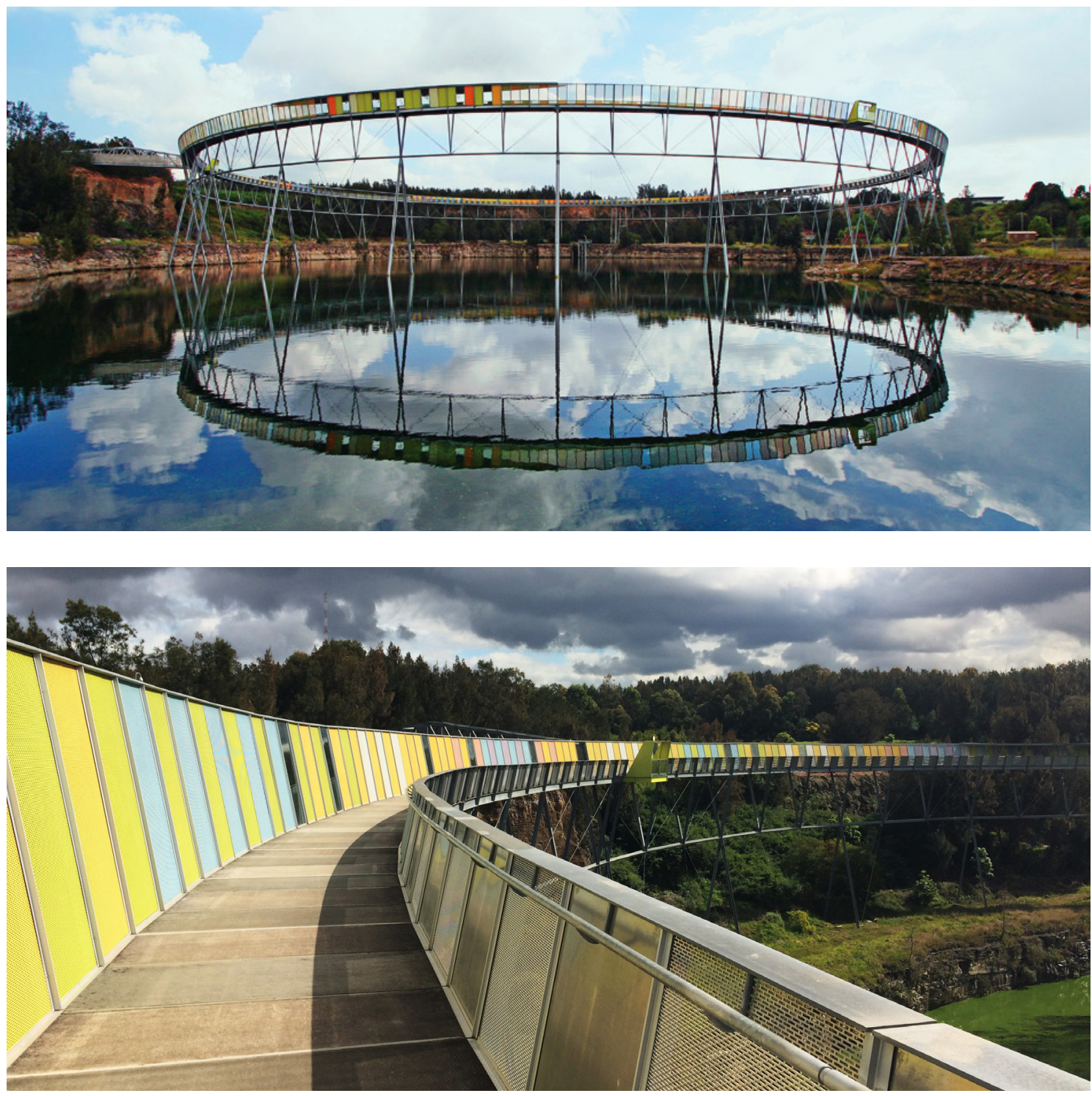

Fig 21.0 Brick Pitt Ring walk (Top)

Fig 22.0 Centre view of Brick Pit Ring Walk (Middle)

Image taken from the ring walkway showing the views into the site from above.

Fig 23.0 Aerial Image of Brick Pit in context (Bottom)

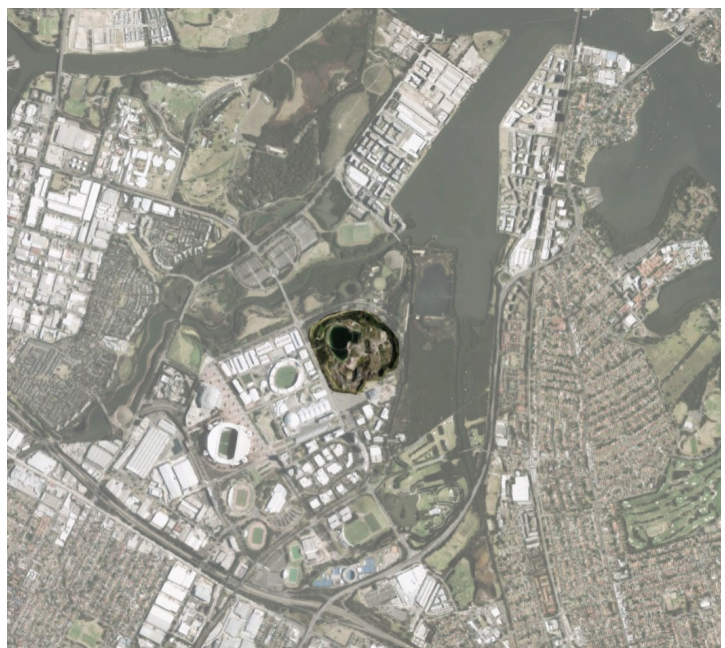




\subsubsection{BRICK PIT RING WALK}

Nestled within a dense urban context, the Brick Pit Ring Walk shows how a precious ecology can be protected, and enhanced through landscape architecture design. The Brick Pit has an extensive history, many of the bricks used in the construction of Sydney came from this site. Present day it provides habitat for the endangered Green and Golden Bell Frog. The main consideration when developing this site as part of the Sydney Olympic park was the retention of the frog habitat. Intervention needed to create a space for people that was not disruptive to the endangered species. "A simple ordering device, the ring walk facilitates both access and interpretation to the brick pit, while fully recognizing its extremely fragile habitat." (Landezine) The intervention uses height to separate human from habitat, a raised circular platform sits above the landscape (Fig 21.0). This allows visitors to interact only by sight and sound, but not by touch.

Protection of the sensitive habitat has been the driver behind this design, the urban context of the site adds to the individuality of the space (Fig 23.0).
The traditional approach to this type of landscape would see the environment shut off from its surroundings, left to function as a standalone system. The approach used for this site however uses the environment as a cornerstone of the design. Allowing people into this space had the potential to be detrimental, however the use of height in the intervention creates a separation between the two functions of the land. The ring walk begins to educate and inform people of the uniqueness of their experience. Using design as a vessel, awareness has been raised for the protection of an endangered species, ensuring its survival.

The vision to enhance a natural habitat through highlighting the function of a site has ensured this design protects the landscape and its natural system. The structure that forms this design is a success due to its minimal impact on the landscape below (Fig 24.0). By creating a raised, artificial ground level this design subconsciously informs visitors how human development, specifically infrastructure, has an invasive and detrimental effect on the natural 


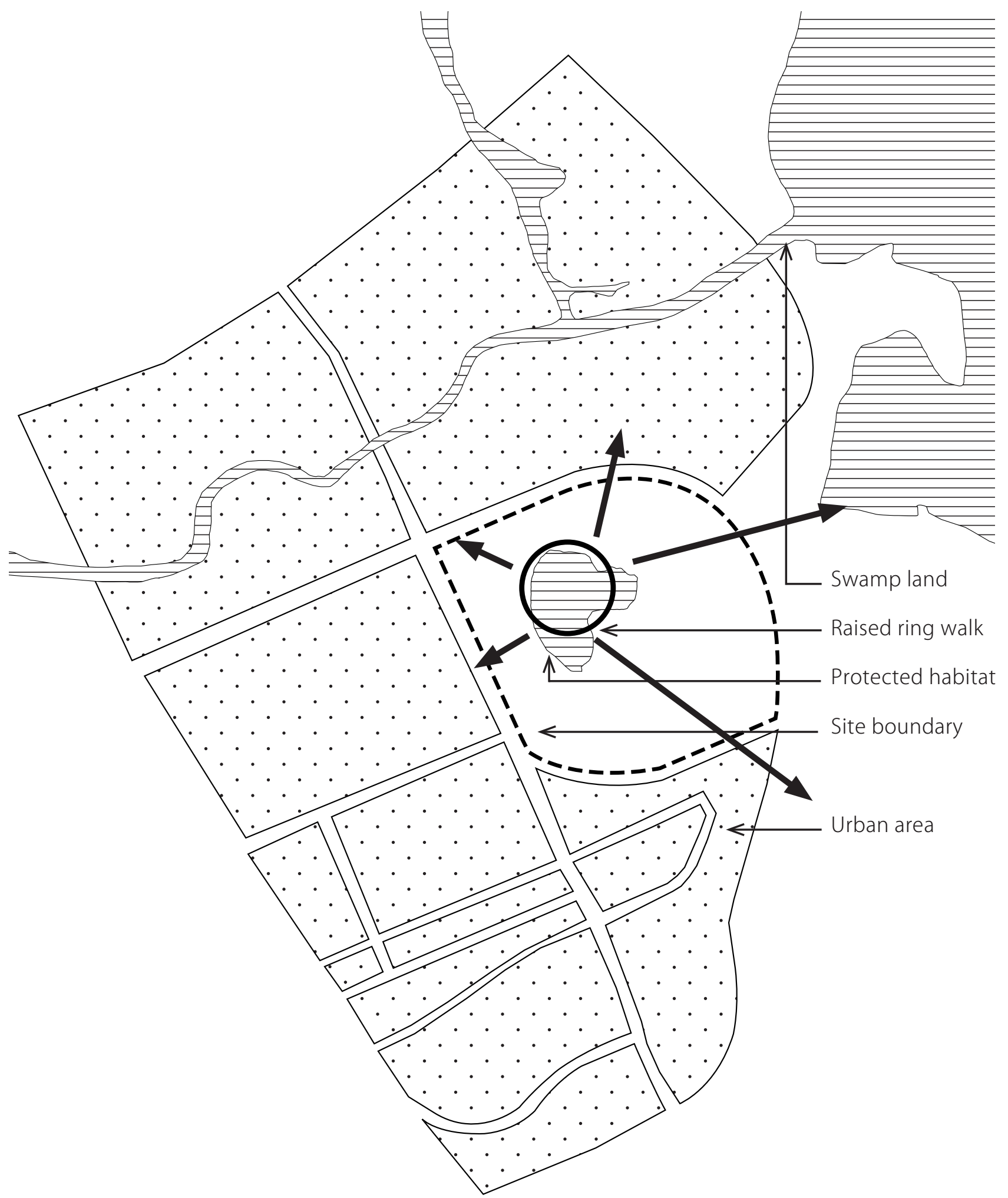


landscape (Fig 22.0). By designing alongside the natural environment a unique level of symbiosis is achieved between the local people of the site and their environment. This Brick Pit Ring Walk is successful in creating respect between people and the land through design, an achievement which many public spaces fail to meet.

\section{Fig 24.0 Brick Pitt Analysis Diagram}

Highlighted are the views outward from the site that seamless integrate the sensitive environment into the urban context. 

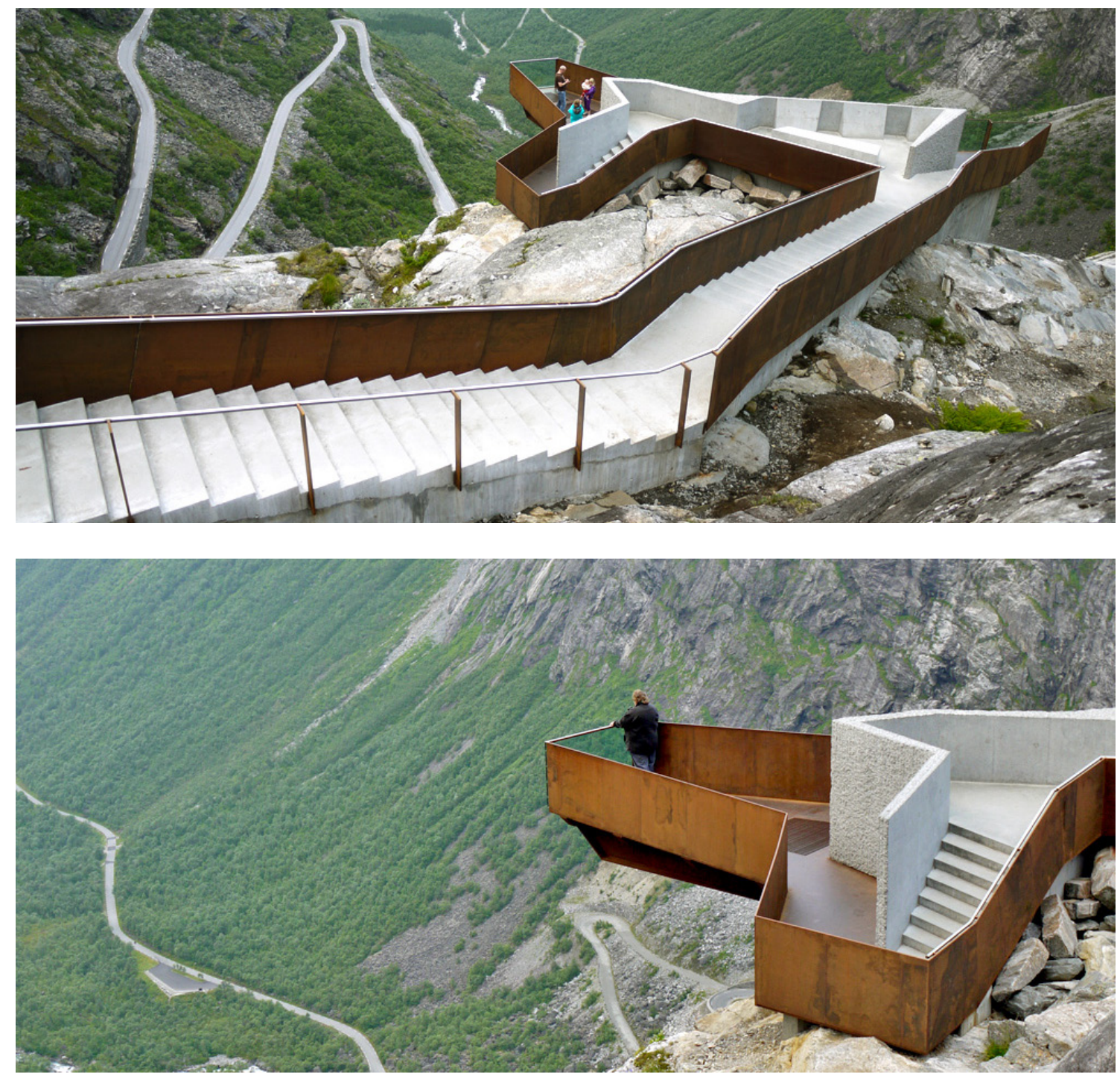

Fig 25.0 National tourist route intervention (Top)

Fig 26.0 National tourist route view platform (Middle)

Fig 27.0 Aerial of national tourist route (Bottom)

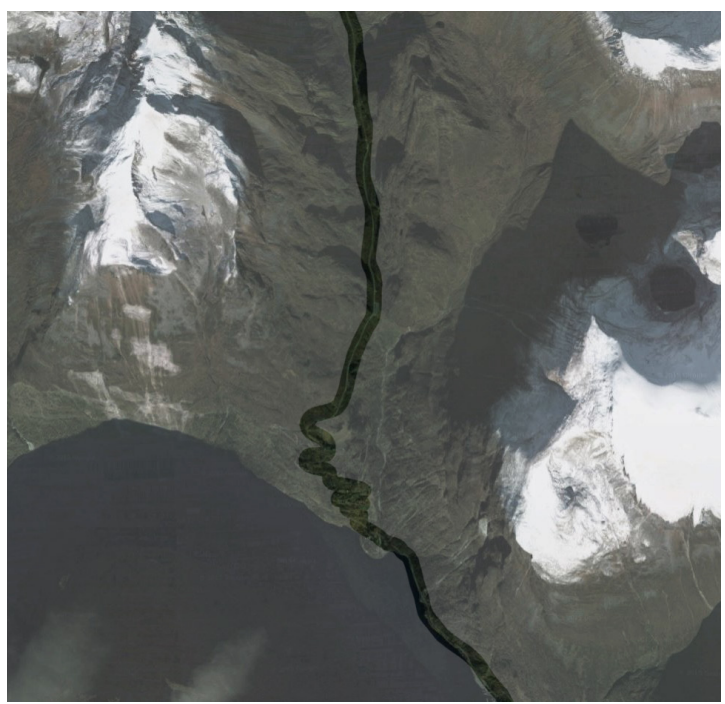




\subsubsection{NATIONAL TOURIST ROUTE}

Complementing the rugged mountainous context Trollstigplataet; National tourist route has been designed to allow people to access to difficult sites, ensuring experience is not lost and harmful effects on the landscape are spared (Fig 27.0). High visitors numbers can degrade the natural environment. The Trollstigplataet design controls how visitors navigate through the landscape in a very controlled fashion. Still providing the unique experience but only taking a minimal toll on the environment. The series of intervention pathways and platforms magnify the scale of the landscape, focusing on the naturalness of the site. Clear transitions between the natural and built allow specific areas of natural sensitivity to be identified by visitors (Fig 25.0). Visitor foot traffic is kept away from the function of the natural system through an elevated walkway, allowing both people and nature to coexist in one space respectfully.

Mirroring the zigzag form of the road that provides access to the site, Trollstigplataet consists of a series of walkways and viewing platforms that fit seamlessly into the wider natural mountain environment. Constructed from carefully selected materials such as corten steel, stainless steel and textured concrete, the detail within the materiality create the sense that the intervention is a byproduct of nature. Each intervention is elevated above the natural landscape, ensuring the native function beneath is retained. Visitors are subconsciously controlled as they move through these zones. The natural hydrology of the site has a significant effect on the design, the dynamic flux of the water throughout the year creates a varying interest; frozen in winter and flowing in summer. Vast amounts of rocks and rigid structures provide a static, more permanent element to the design to retain some consistency.

When designing with nature, the smallest alteration to a system can cause adverse effects. Although this design has as minimal impact on the natural environment as possible, little effort is provisioned to environmental restoration. An effect to not only protect, but enhance this environment would achieve a much more resolved outcome. 


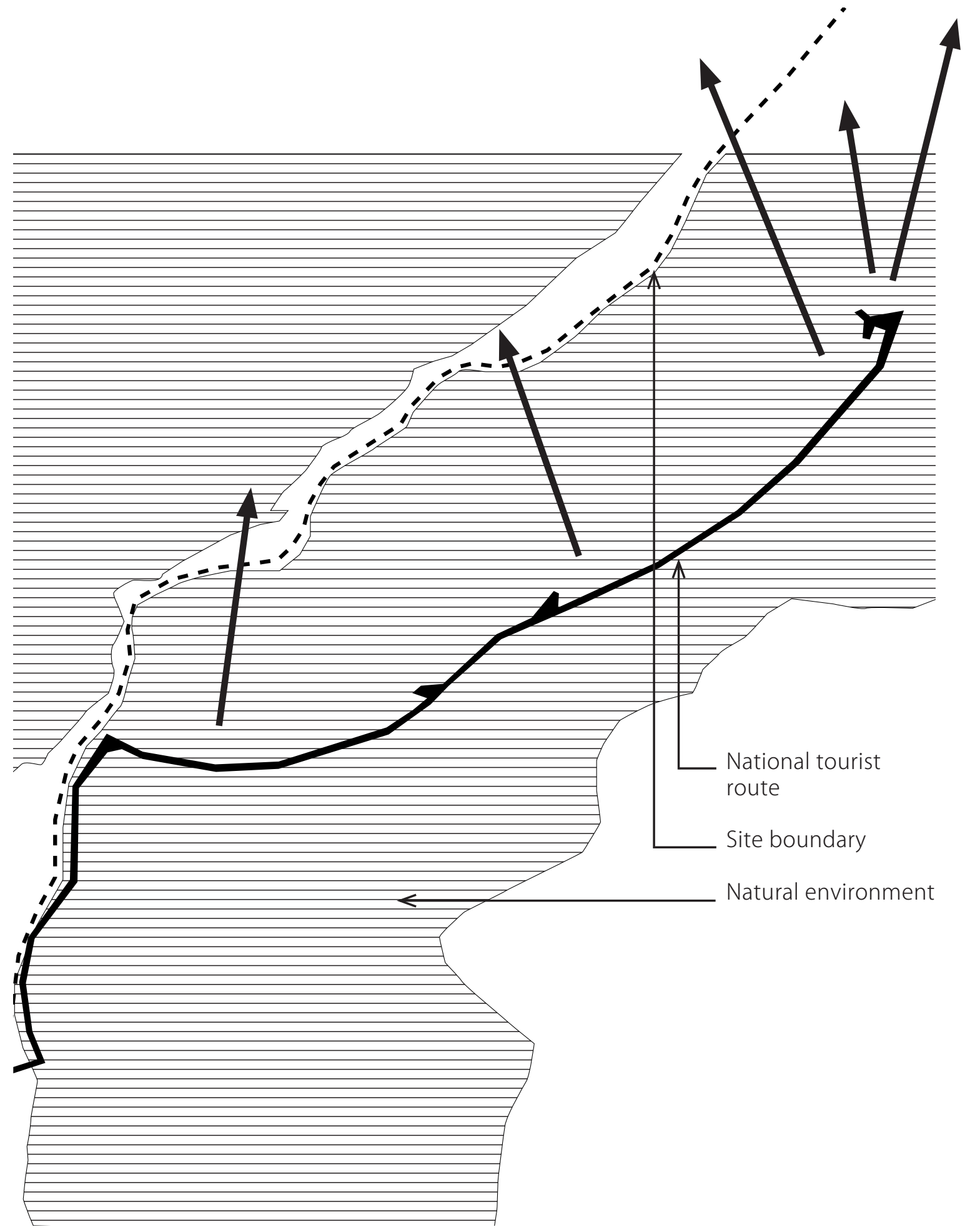


A large amount of road infrastructure cuts through this mountainous terrain, an unavoidable feature of this site. The incorporation of design elements that mitigate the effect of such infrastructure has on the landscape, at a regional scale, is absent from space. Often overlooked, the impact specific sites have on a region bridges the void between a 'designer ecology' and a functioning natural system.

The restraint used in this design to identify which locations require intervention, and which can be left to interpretation, allow the space to function as a vibrant landscape. (Fig 26.0) Moments of intensive intervention create the distinctive transition between the sensitive areas of the natural system and the more resilient without obvious notions such as signs and information boards. Visitors can interact with the landscape, experience its power, and respect the natural amenity it provides all from the safety of this intervention. The attention to detail reflects the natural character of the landscape, materials blend seamlessly with the terrain while still allowing people to realise the transitional nature of the design. Throughout the site, a coherency of form and experience create a vibrant space for visitors while allowing the environment to function without interruption or artificial manipulation. A precedent for design that shows how heavy infrastructure can have a light touch on the landscape (Fig 28.0). A success due to the environment being considered as a dynamic element that is to be designed for, not against.

\section{Fig 28.0 National Tourist Route Diagram (Opposite)}

Highlighted are the views outward from the site that allows visitors to experience the landscape while not damaging it. 



\subsection{PROGRAMME ANALYSIS}

When dealing with a regional site, moving between scales for analysis and design are imperative in the overall success of the outcome. Often overlooked, the medium scale deals with the larger context of a detail site, in reference to regional implications. To ensure a detailed site approach responds to its immediate surroundings a medium scale programme analysis is required. This analysis identifies the opportunities that already exist in the wider context of the site, and how these can be used in both detailed site design and regional design respectively.

Land use on the lake shores and surrounding landscape of Wairarapa Moana varies significantly. Intensive dairy farming adds significantly to the pollution of the hydrological system. Sewage discharge from the neighbouring town of Featherston also enters this system. Detrimental recreational activities around the region are established in many areas. An opportunity for this research to address these issues through landscape architecture is present. This analysis phase will identify aspects of the surrounding landscape that will ensure the design interventions are responsive and site appropriate. 


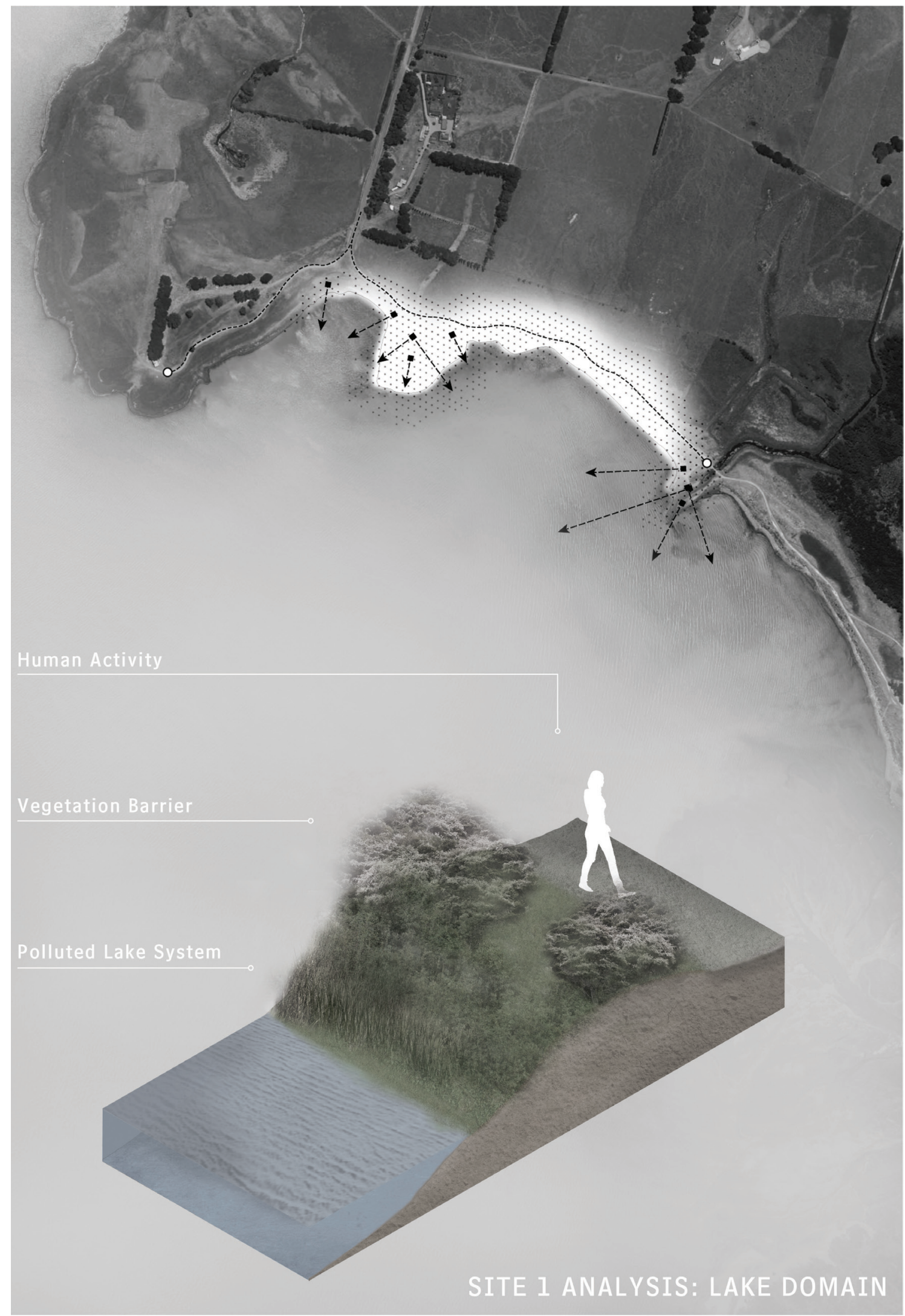




\subsubsection{DETAIL SITE 1}

Located with closest proximity to the township of Featherston, Lake Domain is one of the most visited of the three sites. The lake shore of this site hosts a clash of natural landscapes and disruptive recreation. Alongside polluted streams, Lake Domain also contains a sensitive smaller pond that is a hub for native biodiversity. Although under threat by the neighbouring recreational activities this site has an opportunity to create a gateway to the following two southern sites explored through this design research.

At the Northern tip Lake domain is an entry point of Wairarapa Moana, its proximity to main traffic routes results in ease of access to passers by. The site currently is surrounded by farmland, little organisation of the site leads to unappealing first impressions. Promoted as a recreation hub with amenities to suit a range of needs, this site could set the high standard of experience that Wairarapa Moana has the potential to offer.

Intensive dairy farming is a necessary but degrading use of land, especially the sites bordering Lake Wairarapa. The mitigation of the negative effects caused by such land use is almost non-existent. The potential for design to lessen these effects and explore a synergy between the two spaces is waiting to be utilised. Pollution to the hydrological system as a result of this farming practice happens in many ways. The runoff waste from farms into streams and water bodies can be easily controlled with natural planting in specific areas and better management of our landscape. By assessing the landscape and allocating more appropriate areas for different land use problems such as dairy farm runoff and motocross tracks through remnant wetlands, the natural landscape could thrive alongside its human users. (Fig 29.0)

Fig 29.0 Graphic analysis of Site 1(Opposite)

Site views and human scale obstructions that separate visitor from lake at site 1 


\section{SITE 2 ANALYSIS: WAIRIO BLOCK}

\section{Wetland Restoration System}
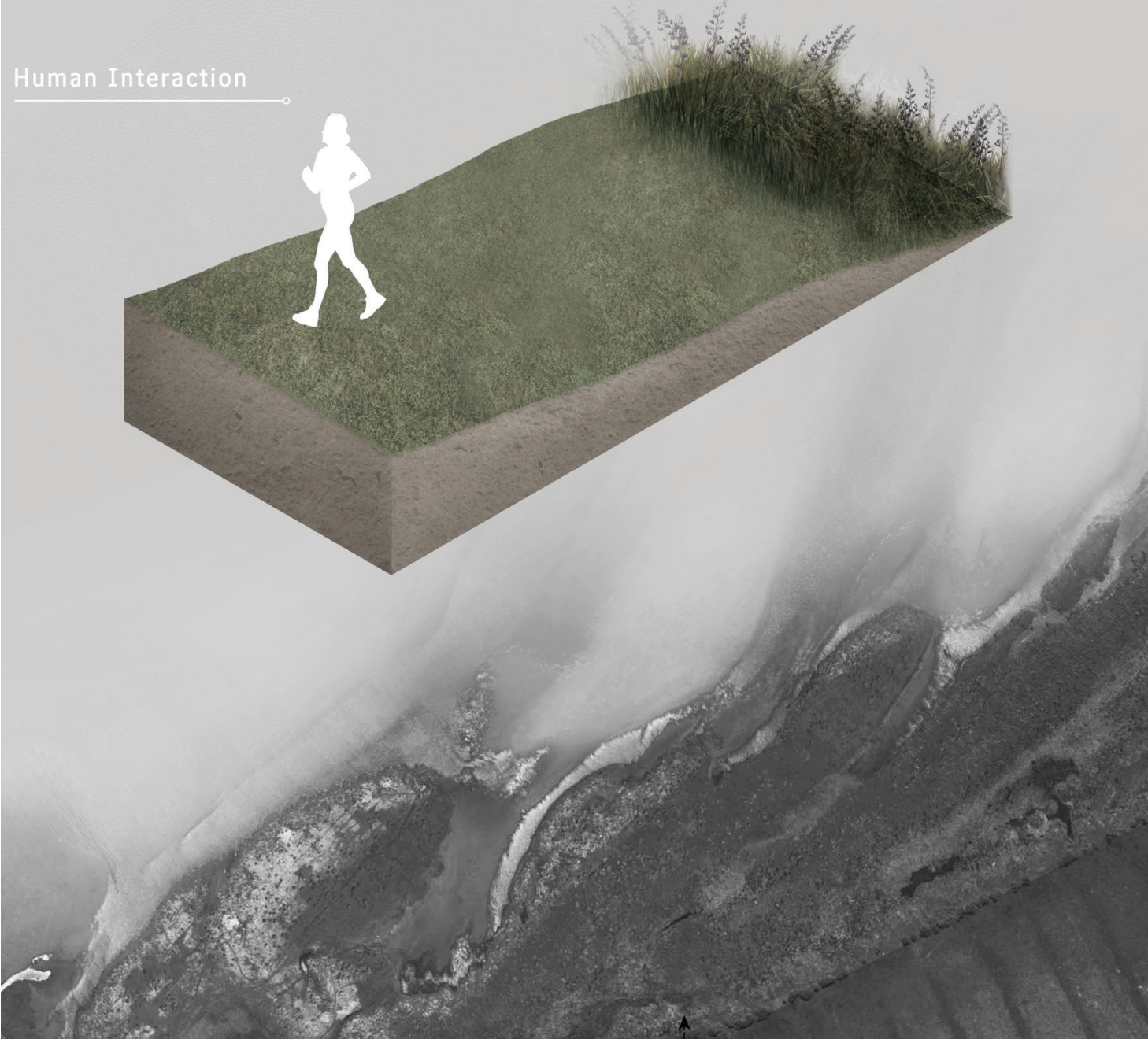


\subsubsection{DETAIL SITE 2}

Filled with biodiversity and native wildlife, Wairio block is bursting with potential to become a vibrant landscape. Many opportunities are present within this site. Wetland function is already tested as positive for this land, further development could push this idea to form a new typology of functional landscape. Currently a weak relationship with surrounding sites sees Wairio block overlooked by recreational users.

Wetland restoration has already begun on Wairio block, lead by ecologists the landscape is well suited to this type of land use. The natural amenity of the site is underutilised and hidden from the public. Landscape architecture has the potential to push the existing wetlands further with the use of design in order to reduce the impact of the surrounding farming practice wand create a diverse open space. Accessibility and awareness are aspects of the site that are lacking; the surrounding farmland blends with the site so much that it's limits are not easily defined. Underlying potential is waiting to be explored through design intervention; neighbouring recreational sites already have an established reputation. Mathews Lagoon and Boggy pond are mere meters away, both with a strong identity as water filled landscapes. Providing walking tracks throughout, Wairio could build on this existing recreation to create a hub of activity on this site, and the surrounding area.

Used as a recreational landscape, this site could also ease the strain on the larger hydrological system by beginning to regenerate the landscape degraded by surrounding farmland. Wetlands working alongside humans, the neighbouring established recreational areas only add vibrancy to this site. Together the surrounding landscape caters for all visitors needs while simultaneously mitigating the negative impact of intensive dairy farming. A true asset to Wairarapa Moana. (Fig 30.0)

Fig 30.0 Graphic analysis of Site 2(Opposite)

Limited views and human seperation between site 2 and lake water 


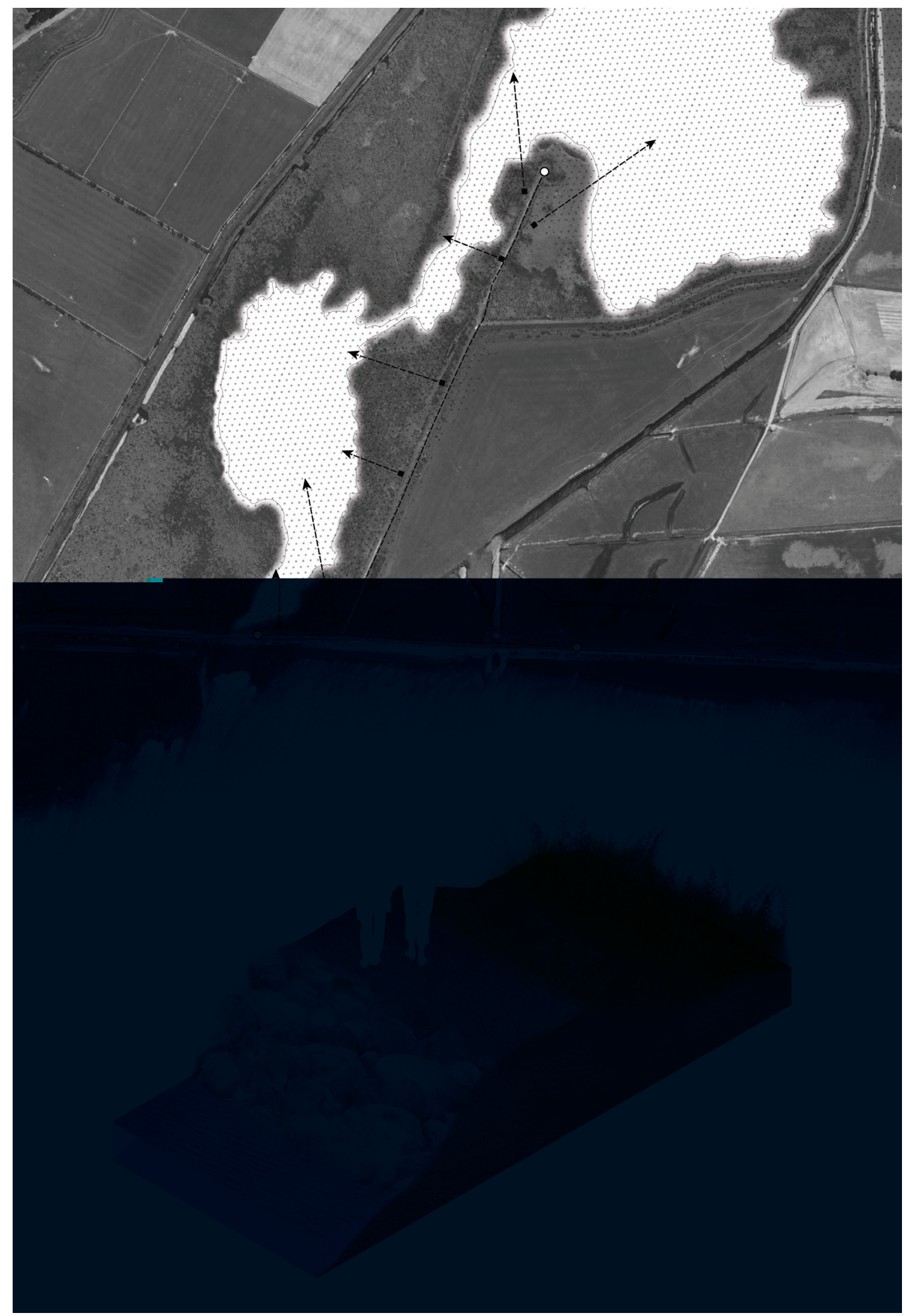




\subsubsection{DETAIL SITE 3}

A site that is in the early stages of regeneration, Pounui lagoon's remote location provides an escape from nearby urban areas. The ranges that border the lagoon create a unique experience where native lowland forest meets the waters edge. Recognised as having significant biodiversity value, intensive dairy farming that once shared the greater context of the site has been shifted further north. Runoff from this land use still enters the hydrological system, however it no longer directly enters the lagoon. The remoteness of Pounui Lagoon paired with its existing amenity value creates a string argument that this site is perfect for design testing.

Protected by the Rimutaka ranges to the West, isolation from the surrounding world is achieved almost instantly upon entering this site. The context of the site also has a remoteness as a small rural road provides the only access to this standalone landscape. The practice of farming that once bordered this site has ended leaving a natural recovery to take place. A significant part of the Wairarapa Moana hydrological system, Pounui Lagoon's wetlands provides water treatment as well as public open space. Limited access for local people has left this site with a negative connotation that requires resolution. A scenic refuge from nearby Wellington City, this site already has natural amenity value that would hugely benefit from further resolution through design.

The remote feeling created by this site is something that design itself cannot achieve so easily. Developing this as a feature the site works in harmony with its natural surroundings to benefit the landscape. Design has the ability to make this site more user friendly for people, encouraging more visitors and value to the landscape. The possibility of forming a stronger relationship between landscape and humans has the potential to blossom at Pounui Lagoon. (Fig 31.0)

Fig 31.0 Graphic analysis of Site 3(Opposite)

Limited views and no interaction between humans and the natural amenity of site 3 

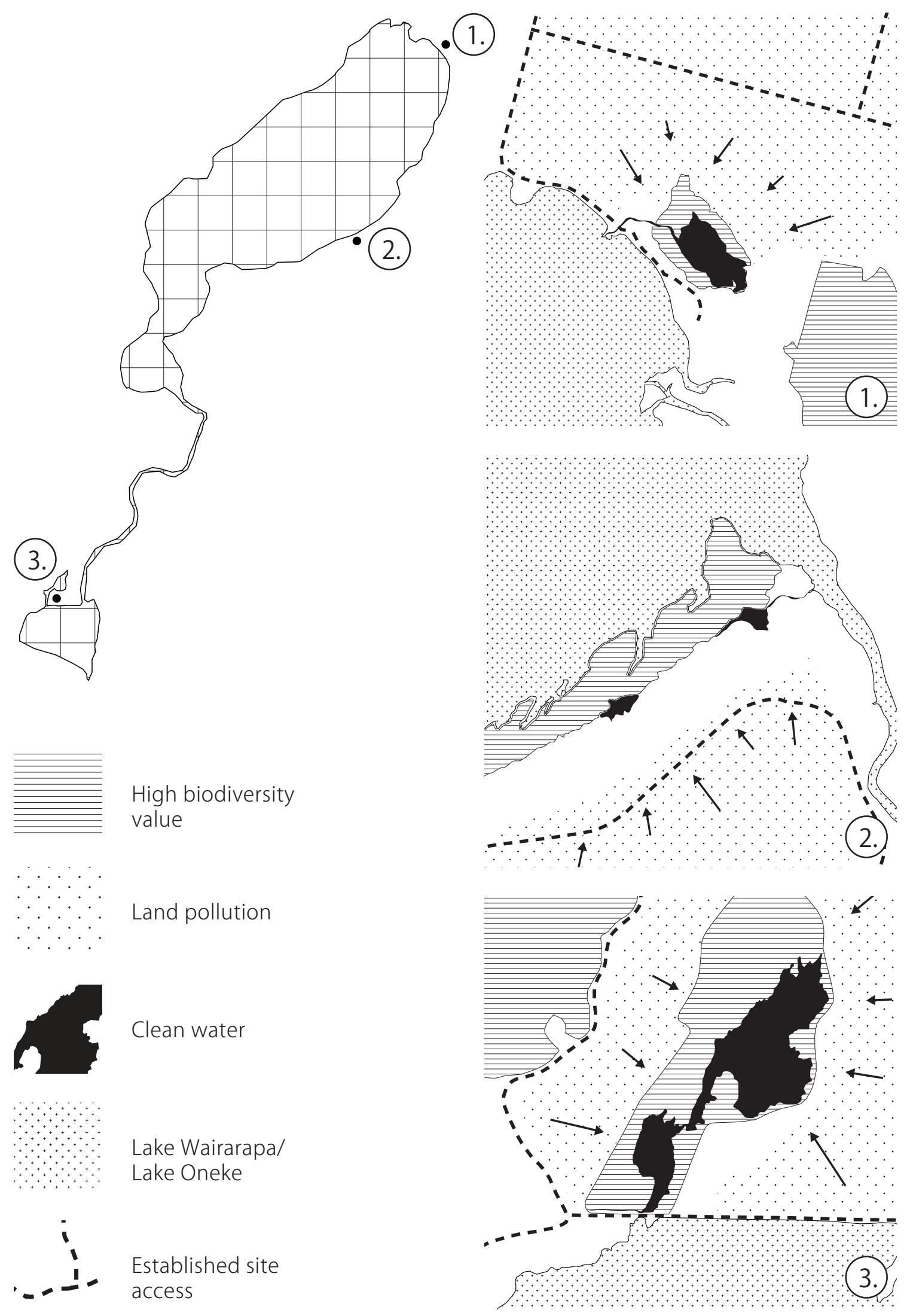


\subsubsection{REGIONAL CONCLUSIONS}

The mid scale of a regional landscape refers to the surrounding land of a detailed site. In South Wairarapa this mid scale landscape is an important consideration as much of the pollution effecting the hydrological system is identifiable on each site.

A combination of biodiversity value and land pollution come together at this scale, evident at each of the three selected sites for design. Designing at multiple scales identifies a multitude of issues that require resolution. Site two in the context of this research identifies as the site with the largest disconnect between biodiversity, land pollution, proximity to Lake Wairarapa and access. (Fig 32.0) For this reason site two has been selected to move forward in more detail to achieve a resolution of design that could be applied to the other sites later in the progression of design. Sites one and three both have aspects of the criteria present, but they are not as prominent as site two. (Fig 33.0) (Fig 34.0)

Conclusions about design principles are also drawn at this stage of the design process. A clear understanding of the most important values of this site have been gained. Design principles established will progress this research and provide a framework for evaluation. The design principles are as follows:

- Resilient spaces that adapt to the needs of both people and the land as a natural flux progresses on with time.

- Open space that affords both environmental infrastructure and public space.

- Restorative design that protects and/ or enhances the value of the land for the future.

- Financially feasible public space design in South Wairarapa.

Fig 32.0 Mid Scale site 1 (Opposite, top)

Fig 33.0 Mid Scale site 2 (Opposite, middle)

Fig 34.0 Mid Scale site 3 (Opposite, bottom) 


$$
\text { DESIGN }
$$




\subsection{DESIGNED RESILIENCE}

How can our landscape be designed in such a way that a new, multifunctional typology is created? A typology that is inclusive of both open space and green infrastructure working harmoniously as one system. The consideration of how such a space might function to meet the needs of both human experience and infrastructure functionality is the key driver for this research. The design outcome will challenge how the water quality of Lake Wairarapa can be improved while strengthening human relationship with the landscape. Design drivers including economic feasibility, experiential qualities and functionality require careful resolution to achieve the overall goal of multifunctional open spaces. The creation of a unique experience that draws people to this landscape is an imperative aspect of a successful design, not only residents of the South Wairarapa but visitors to the region. 


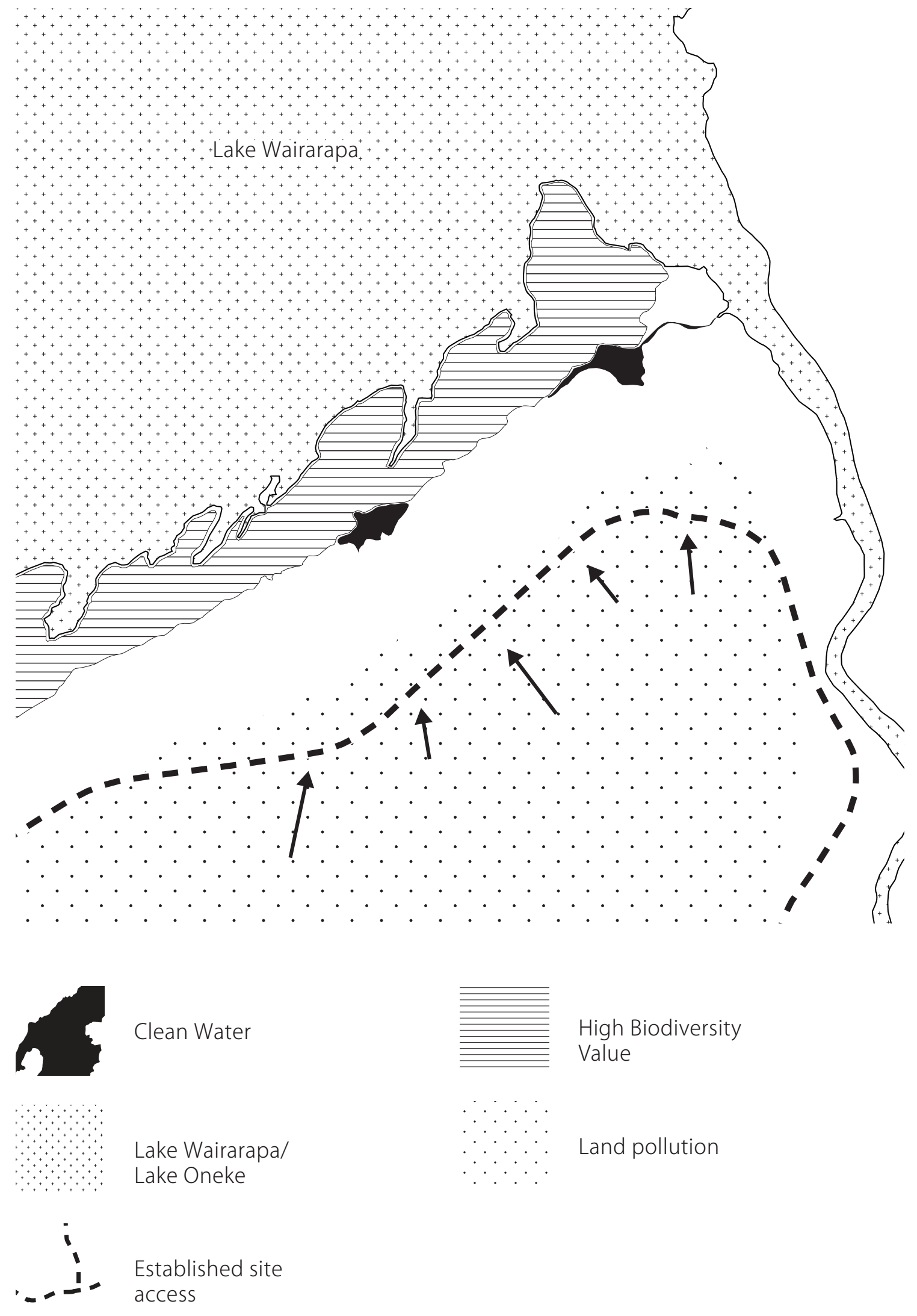




\subsection{PRELIMINARY DESIGN}

The South Wairarapa district covers a land area of just over 800,000 hectares (id). Designing for impact of such a large site requires multiple scales to be used simultaneously. To ensure the research conducted is representative of the South Wairarapa, each of the three sites identified in analysis were evaluated against each other. The conclusions drawn highlighted which site posed the highest number of challenges in terms of clean water, access, biodiversity value and pollution. Site two has been selected for design testing to allow a more rigorous and resolved design to develop through a multitude of scales. The design solutions and principles gathered from Site two could then be applied to the remaining two sites, giving a resolved design outcome that is best suited for the regional scale of the overall site. (Fig 35.0)

A weak respectfor the lake has resulted in a poor water quality of Lake Wairarapa's hydrological system. The lake is removed from human contact by a range of obstacles including stop banks, exotic vegetation and limited access. Site two contains the most extreme example of this. Limited access stops human interaction with this natural resource, resulting in a weak relationship.

Small scale intervention from private community groups have attempted to mitigate some adverse effects of activity on the lake shore. Site two also contains Wairio Wetland Restoration, a private restoration project by Ducks Unlimited. Wairio wetland restoration provides an opportunity for this research to use the same key principals but executed on a larger scale through design. The outcome being more inclusive of a positive human experience and rich with recreational programme.

Artificial controlling of the hydrological system is present in the form of stop banks. These further separate interaction with the lake and severely alter the natural function of the few remaining remnant wetlands. By not allowing water to flow in its natural path during flood events, silt and pollution continue to build, degrading the landscape.

Fig 35.0 Site two challenges diagram 

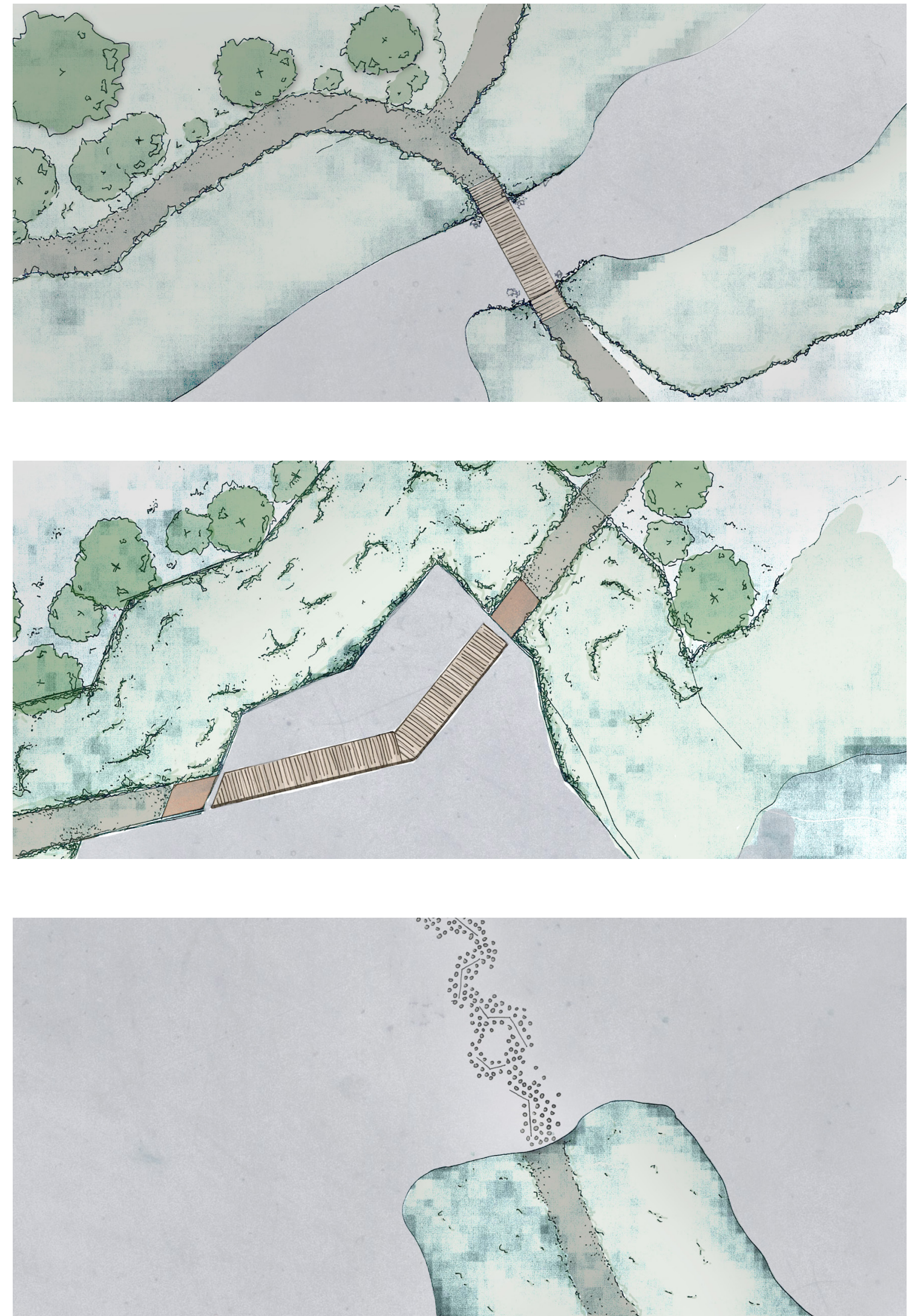


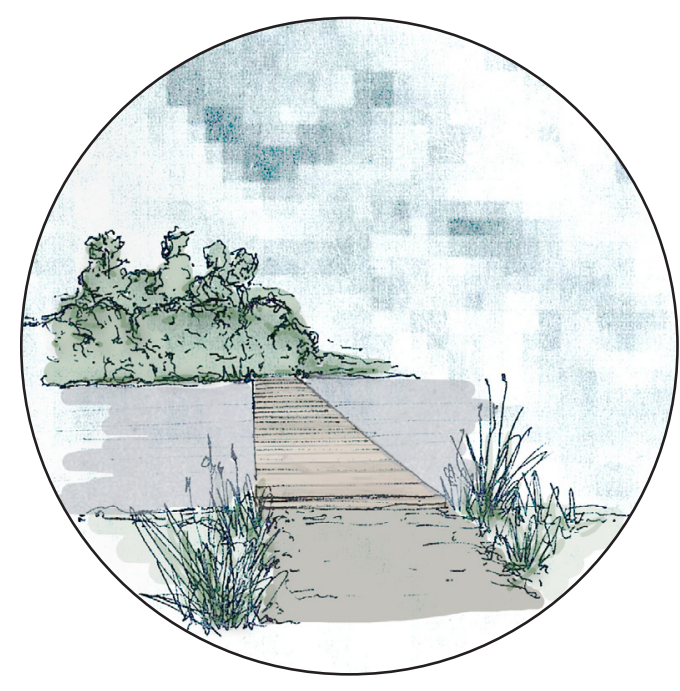

\subsubsection{DESIGN TESTING 1}

The initial design test 1 uses the core function of online wetland restoration to break down the site into small scale typologies that interact with water. As online wetlands have a continuous water flow, interaction with this water is necessary as visitors explore the site and follow the water through various stages of treatment. The limits of the site have been reduced from the analysis stage to focus more on the existing water body, creating a series of more intimate spaces with simple interventions.

Fig 36.0 Entrance views (Top)

Fig 37.0 Artificial nature (Middle) Fig 38.0 Pollution interaction (Bottom) 

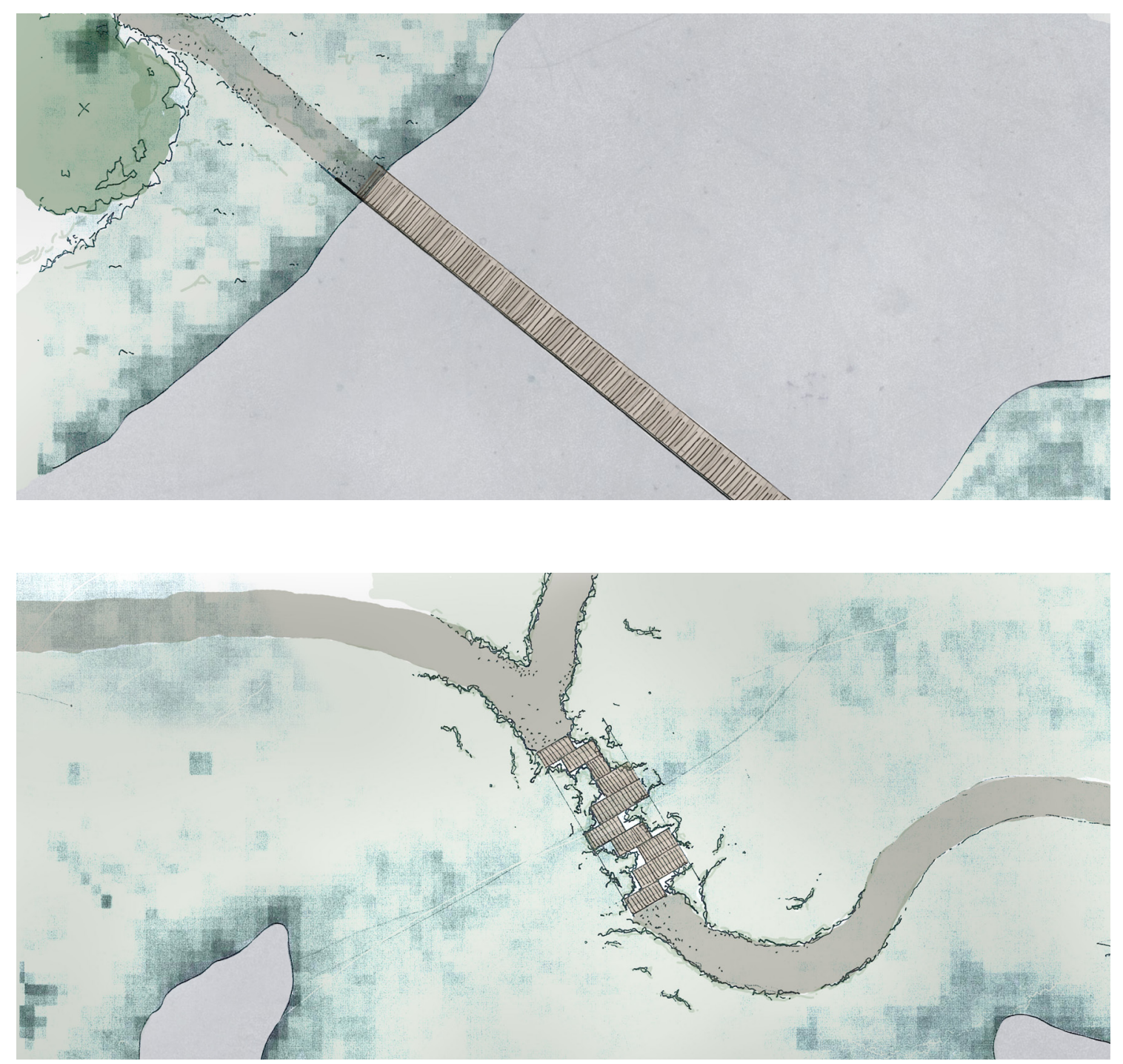


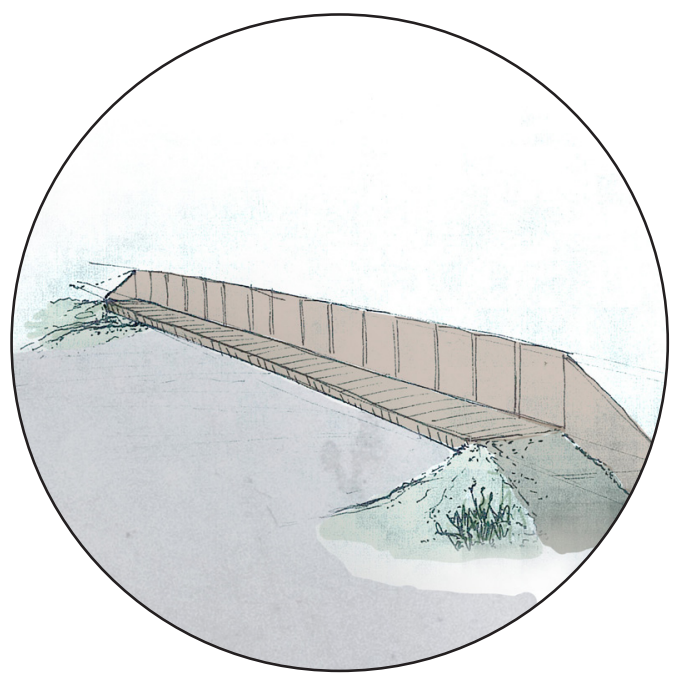

POSITIVES: Low cost design using simple materials in an innovative way. Creating a number of water experiences for a range of people. Light structures allow the landscape to flood naturally without causing debris to build up on structures. The flux of the landscape is allowed for by varying heights and the subtle manipulation of water already present on site.

NEGATIVES: This design test allows people to interact with water, but it does not subconsciously inform about Lake Wairarapa's poor water quality and the inability of people to use the lake for recreation. Visitors to the site are again kept away from the lake shore, human relationship with the land is not strengthened in any way. Very generic looking design, nothing to draw people into the site, and encourage visitors to take some ownership for the landscape.

Fig 39.0 Lake views (Top) Fig 40.0 Environmental preservation (Bottom) 

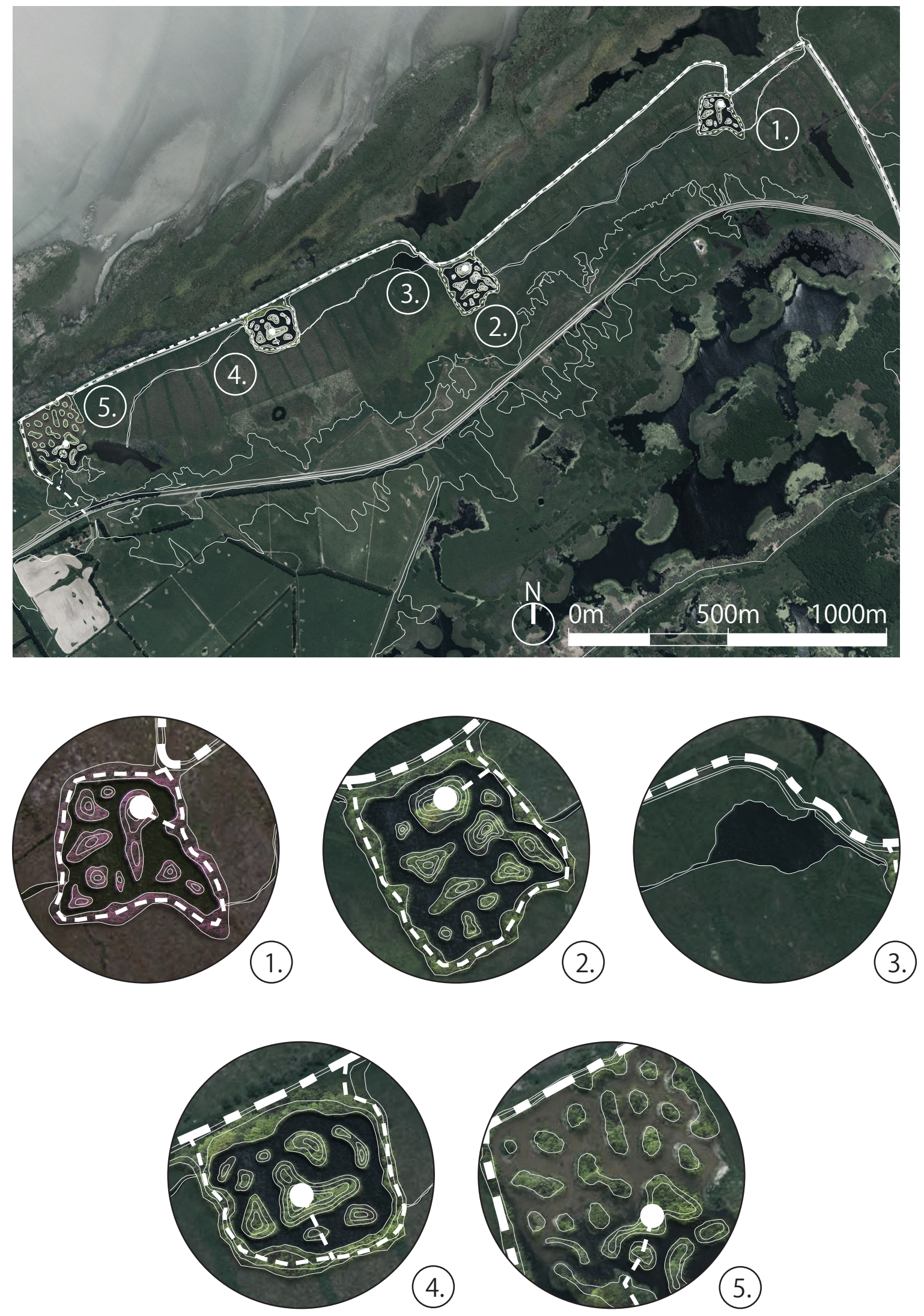


\subsubsection{DESIGN TESTING 2}

Working with the existing features of the site, design test two pushes the boundaries of the established, small scale restoration wetland (Fig 41.0). Building from the previous design test, a series of large wetlands clean the polluted lake water in various stages as it travels along the natural topography. This design allows visitors to share the waters' journey by foot through the use of an elevated walking track situated upon the existing stop banks on site. Green infrastructure shares the space with recreation, encouraging people to form a bond with the landscape as a natural treatment process mitigates the harmful effects settlement has had on the natural environment.

The relationship people have with their landscape is further strengthened by a body of clean water, the central element of this design. The polluted lake water travels through the wetland systems where it is naturally treated and cleaned before entering a small lake that affords recreation and human activity. This allows people to understand the general function of the journey they have just travelled and understand how this system on a larger scale could begin to clean Lake Wairarapa. A long term goal of returning the lake to its former perception as a recreational hub and valuable resource is realised.
Fig 41.0 Site 2 plan (Opposite)

Plan showing initial design iteration for site 2 

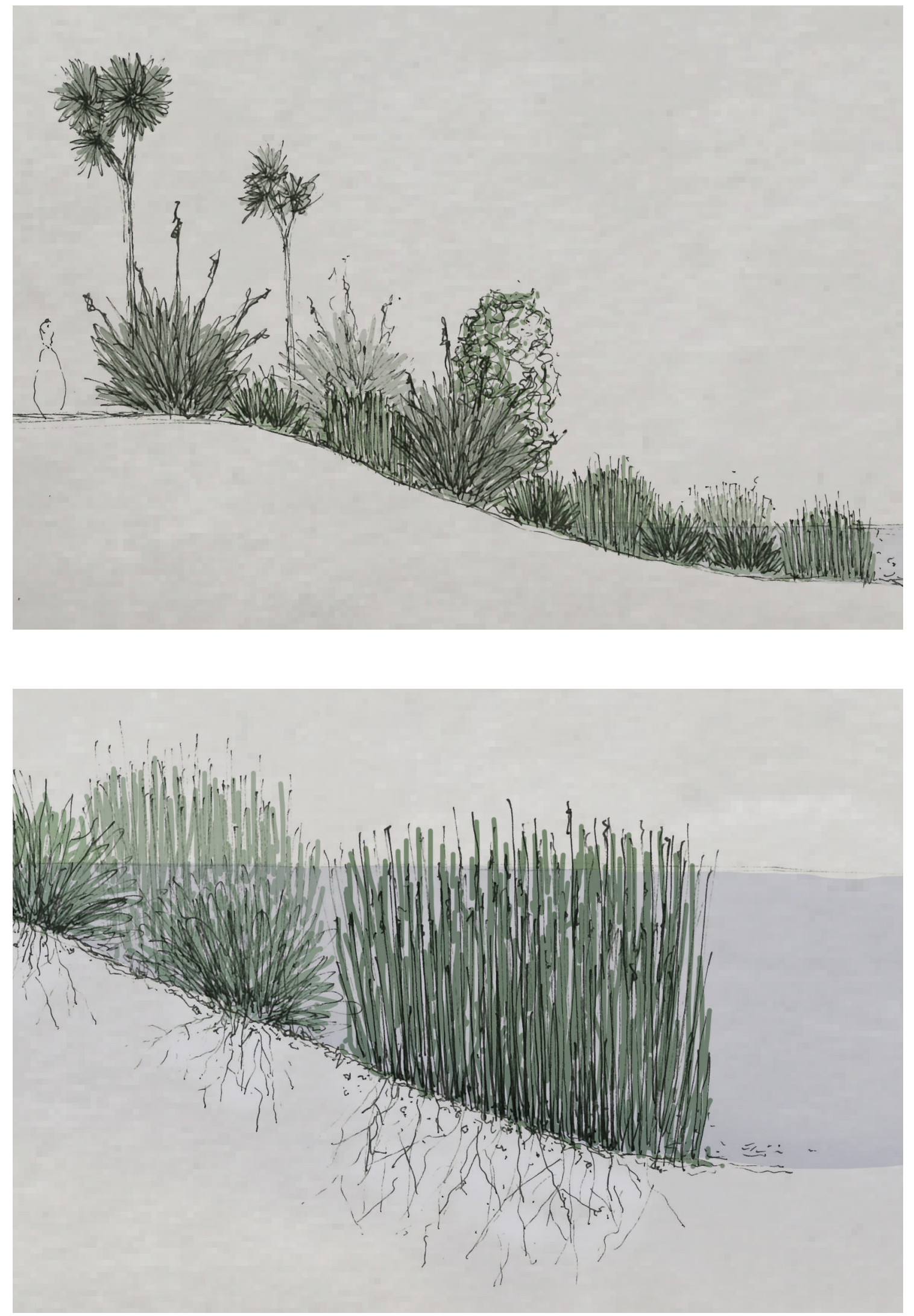
POSITIVES: Green infrastructure is functioning in the same space as human interaction, seamlesslysharing the space. A unique form of interest is created at various stages throughout the journey, each wetland can be explored more as the water progressively becomes cleaner (Fig 42.0). An economically feasible design that makes use of the limited topography on site and creates an intervention using the existing stop banks in an untraditional way.

NEGATIVES:

The planting, a feature of the scheme, creates a very 'typical' New Zealand landscape aesthetic. Design testing two does not contain bold enough changes to show how designed infrastructure can be present on site and have a positive effect on the environment. Missing an element that draws people to site, and keeps them coming back. Little variation in the overall route, needs to cater for a wider range of visitors with different time restraints.

Fig 42.0 Typical section of wetlands
(Opposite, top)

Section cut through a typical wetland in site design testing 2

Fig 43.0 Wetland planting detail (Opposite, bottom)

Typical detail of wetland planting in site design testing 2 

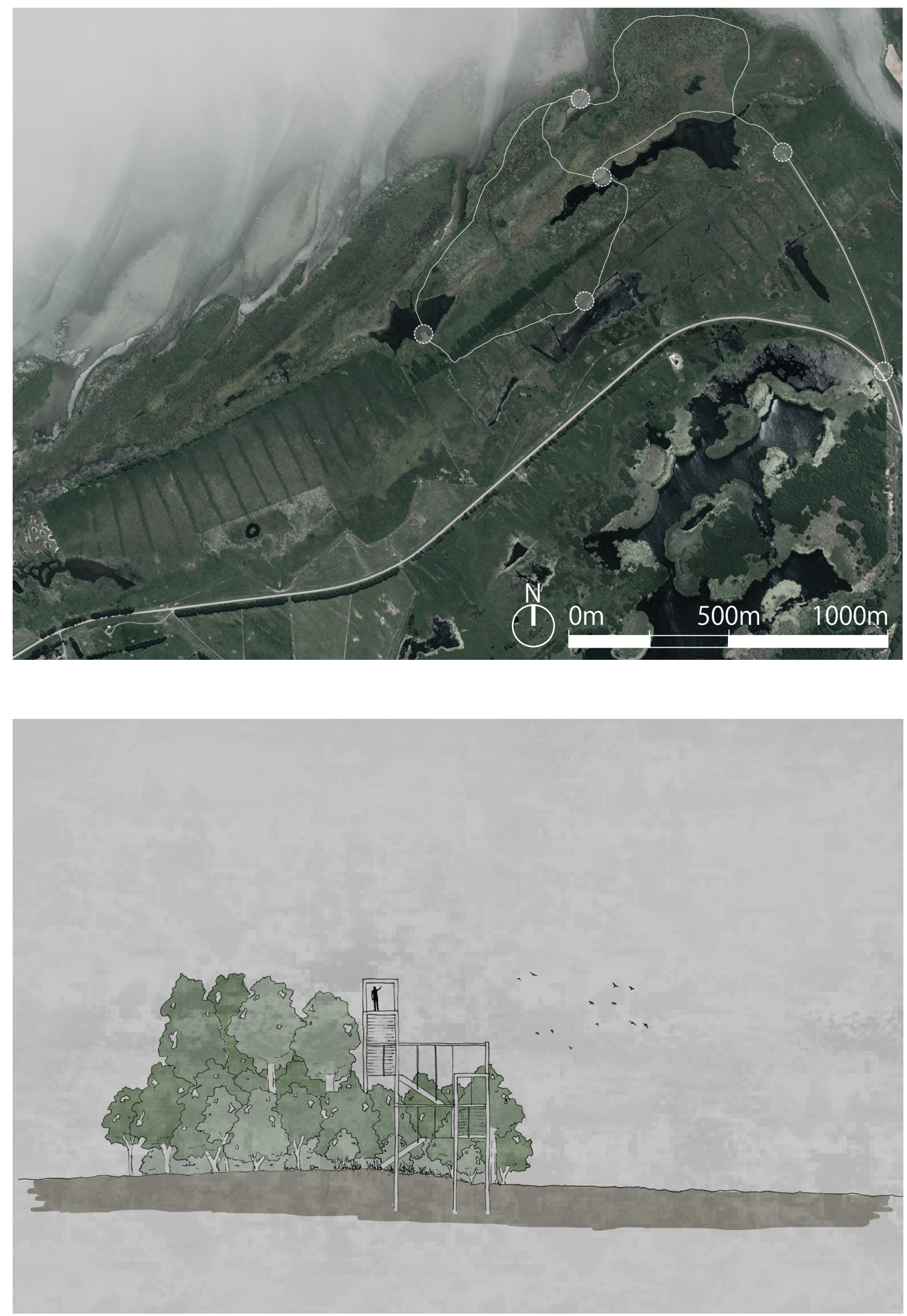


\subsubsection{DESIGN TESTING 3}

Design test 3 uses the element of height to add a vertical element that contrasts the horizontal landscape that surrounds Lake Wairarapa. (Fig 45.0) Lake Wairarapa is seldom viewed from above allowing visitors to view the lake at a certain height as well as gaining a sense of ownership. A minimal stripped down structure that allows nature to share its foot print turns full emphasis to the water and not the intervention. At a height of eight metres above the lake level each of the three sites are visible to one another by the human eye. This creates a visual link over the entire lake system. Attention is given to the pollutants that enter the hydrological systems at various sites, which can be observed from the view platforms. The pollutants are naturally highlighted through a dramatic change in water colour/clarity as the silt heavy water mixes with the clearer water of the central lake.
Fig 44.0 Plan of design test three (Opposite, top)

Fig 45.0 Section cut in design test three (Opposite, bottom)

Section showing the view platform in design test three amongst established planting 

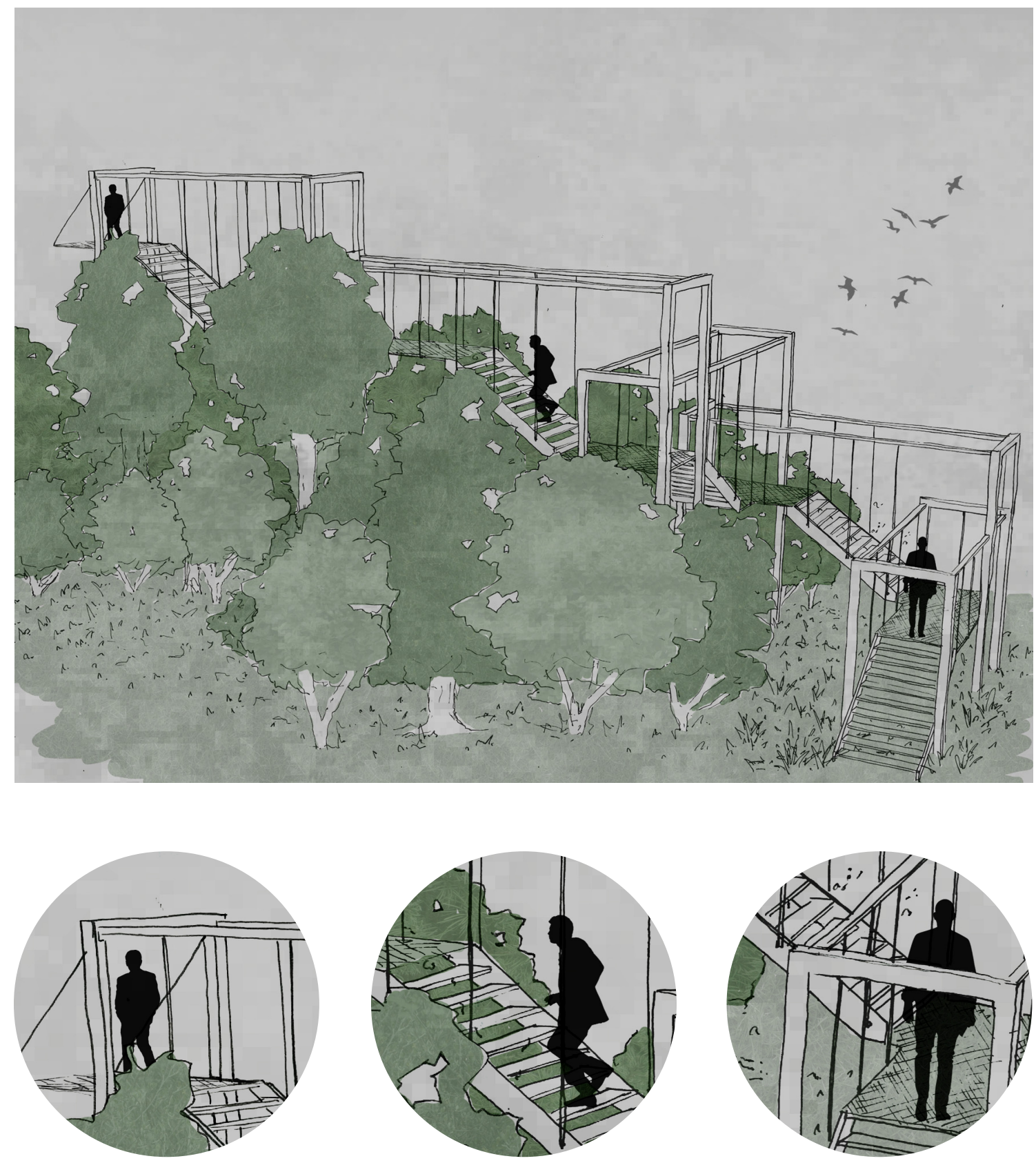
POSITIVES: The use of height adds a unique element to the landscape, while not being too invasive. This type of intervention would draw people to the site. Minimal infrastructure needed to get people to the required height, over time planting such as Kahikatea would grow in and around the structure, blending it with the landscape (Fig 46.0).

NEGATIVES: Little circulation around the site is allowed for, amenity value is not improved by a small section of the site being used. The function of the wetlands to improve water quality go unnoticed, the integration of human experience and infrastructure is not fusing correctly. The relationship between people and the natural environment remains weak as the human experience has all the power by being literally above. 

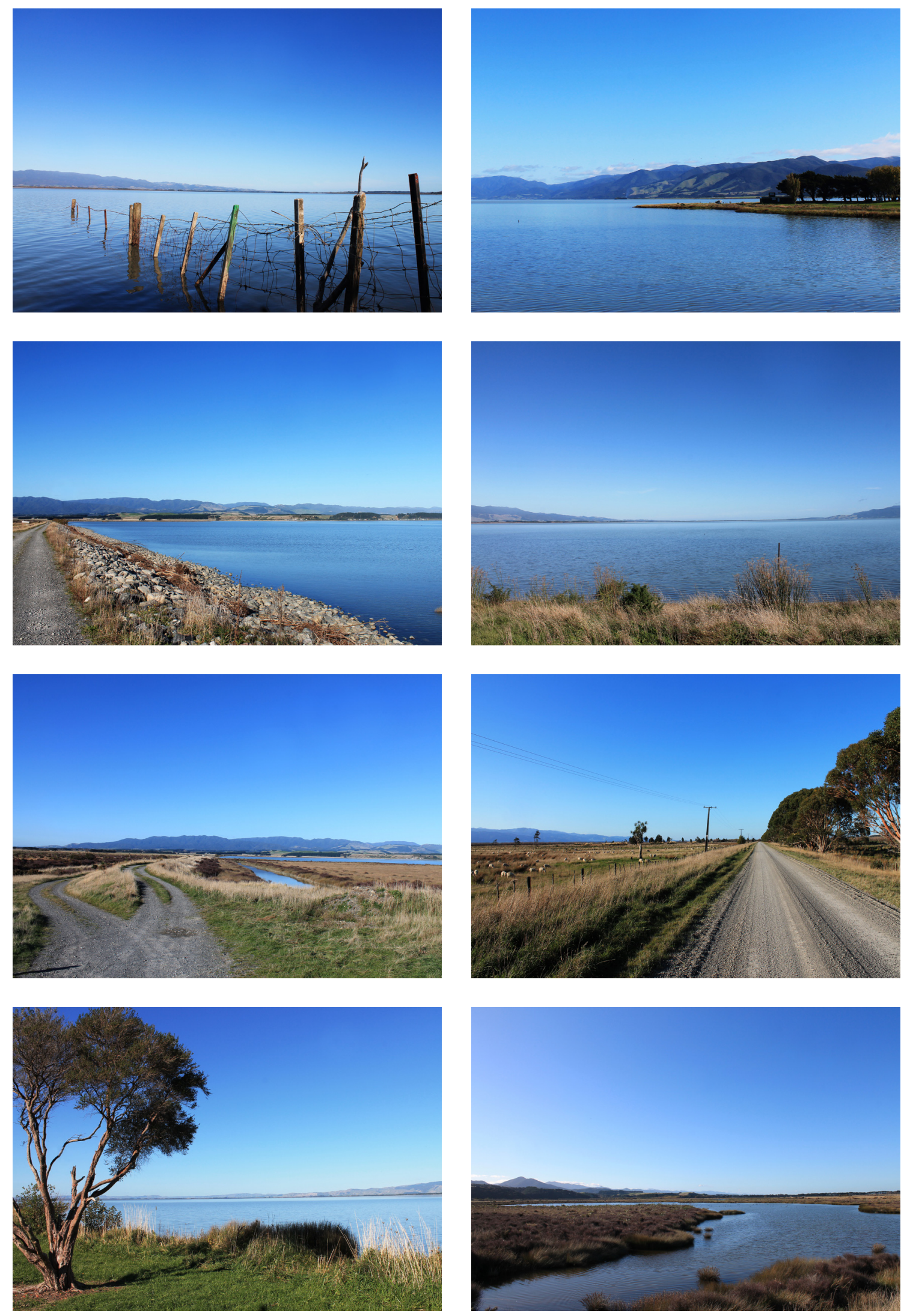


\subsubsection{REGIONAL SITE APPLICATION}

The process of small scale site testing, using site three as a vehicle for this process, resulted in design test two being the most appropriate iteration to develop. The application of the principles gained from site two, are applied to the South Wairarapa region to evaluate the strengths and opportunities of the intervention at a much larger scale. This process of doing so identified opportunities for wetland implementation within the initial design, at both small and large scales, for further development.

The principles established in the analysis phase are continually being used to assess design development. This assessment ensures that the design is following the principles and exceeding the expectations of the traditional landscape architecture approach. Design test two was shown to meet the basic principles, however the primary function of this exercise was to test concepts on site. This leaves opportunity for design evolution to take place as the site is further understood and natural progression takes place.
The conclusions drawn from this process, at a regional scale, found that a more rigorous development would benefit this design research. The process of extracting the fundamental drivers that will create a successful design was crucial for the development of the concept. Hydrology, landscape flux, economic feasibility, infrastructure, amenity, flora and fauna all require a detailed consideration in the resolution of the current issues faced in Wairarapa Moana.
Fig 47.0 Site images giving the context of South Wairarapa (Opposite) 


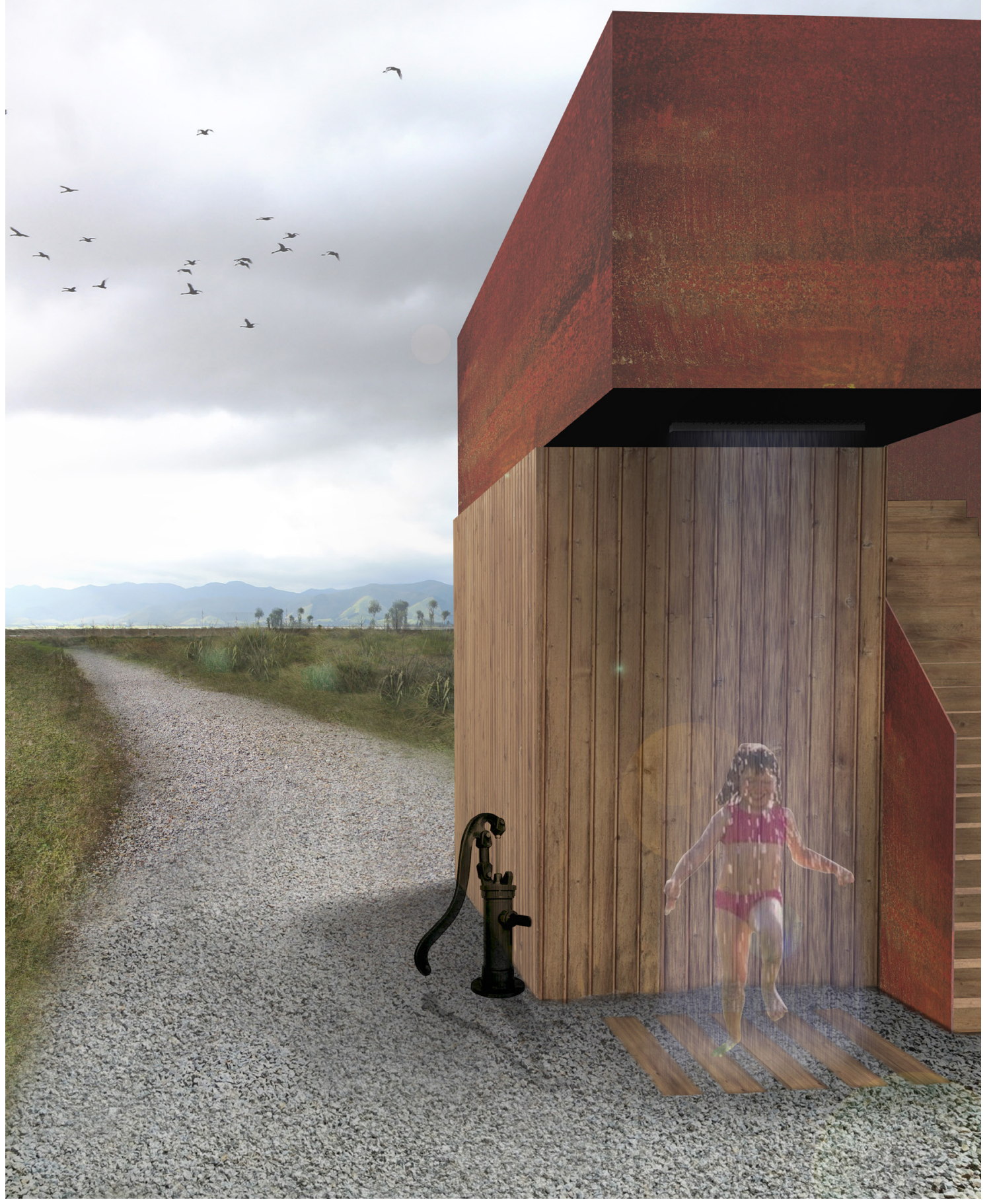




\subsection{RESILIENT LANDSCAPES}

Designing landscapes for the future is unpredictable and one of the largest challenges of the landscape architecture discipline. Ensuring design is resilient in outcome is crucial in the ever changing world. Design must also evolve with the landscape to ensure the most appropriate solution for the environment is reached.

Resilience as a disciplinary tendency typically sees, at most, multifunctional structures and interventions that attempt to adapt to programme when required. This exploration of design aims to push these tendencies further and over a larger time line to achieve a positive change in the health of our natural environment. Further creating a resilient landscape that is not reliant on structures and intervention but rather the natural function of the land to withstand the test of time.

Designed environments have the ability to utilise the natural function of the landscape, working in harmony to achieve resilient public spaces. Reconciliation of a poor relationship between humans and the land is a fundamental of landscape resilience. The resolution of this relationship would see land use being more appropriate for each site. Guardianship of our natural environment is something to be gained from a new formed positive relationship. Advocacy for our natural systems and their protection in the future is a goal that is to be achieved through the use of resilient landscape design, further strengthening relationships between people and the natural environment.
Fig 48.0 Perspective view of final design structure (Opposite) 


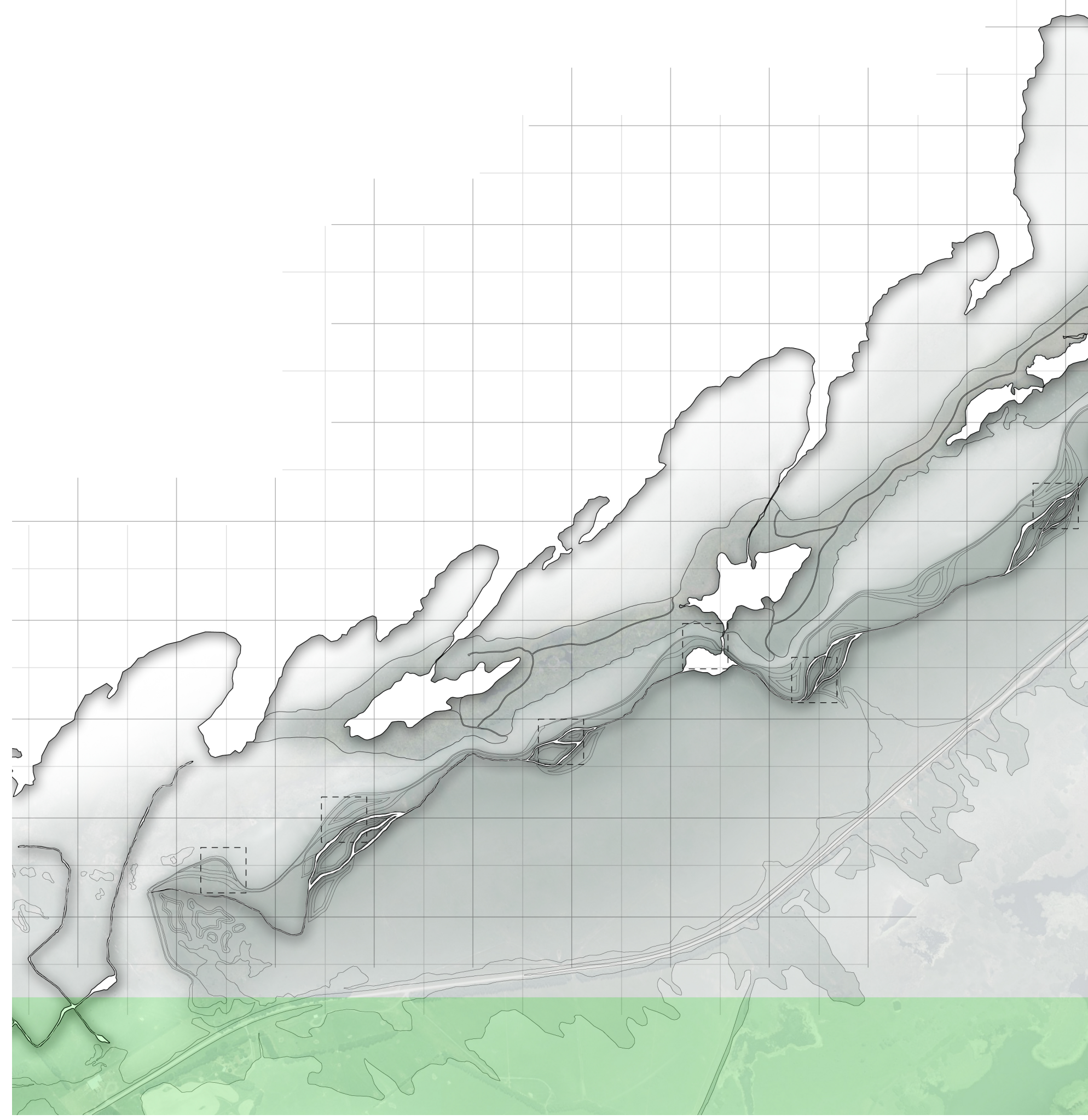



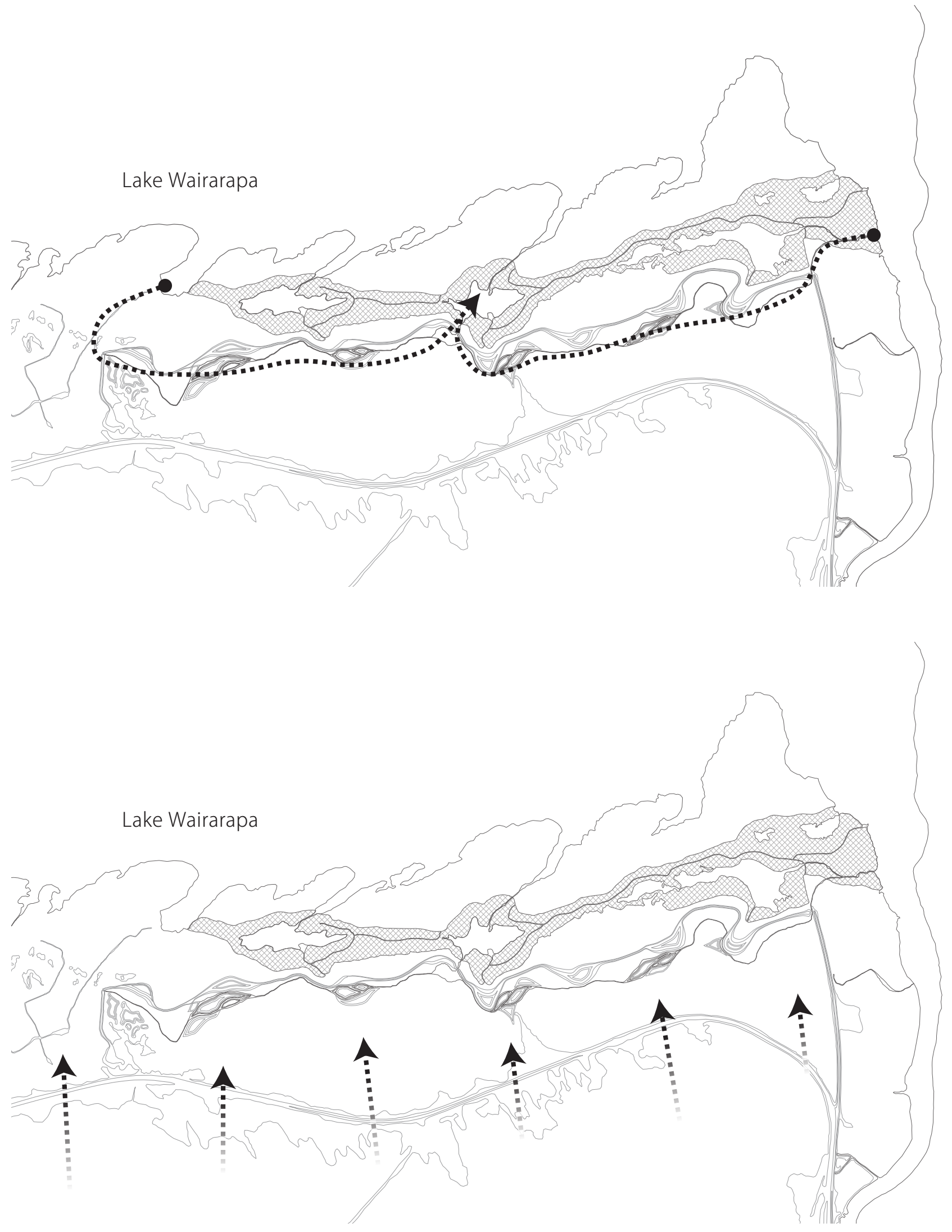

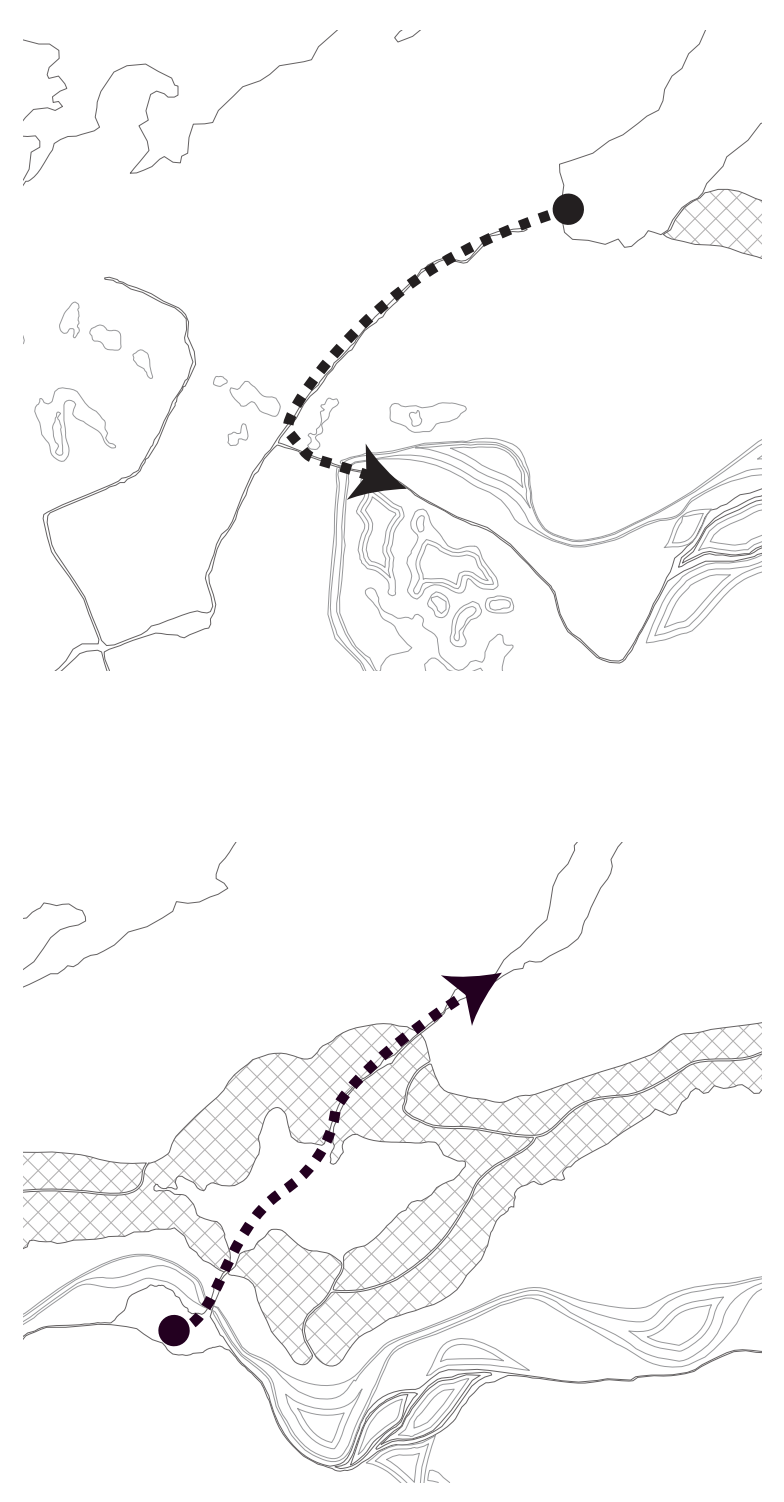

The hydrological system intervention that is a result of this research deals predominately with diffuse pollution. (Fig 51.0) As point pollution can be mitigated at the site of discharge, more awareness is needed for diffuse pollution. This design uses the polluted waters of Lake Wairarapa and Wairarapa Moana's ground water. (Fig 50.0) By combining water treatment infrastructure with public space that has amenity and recreational values, resulting in a new typology for Wairarapa Moana. A typology that not only benefits the landscape in function, but also begins to teach visitors to the site about the importance of water treatment in degraded landscapes. Changing people's perception about the water in Wairarapa Moana is the first step in gaining more advocates for the protection of the natural environment.

Fig 50.0 Site 2 water movement diagrams

(Opposite, above)

Fig 51.0 Site 2 pollutant indication diagram (Opposite) 


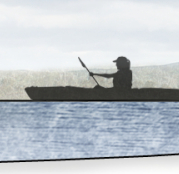



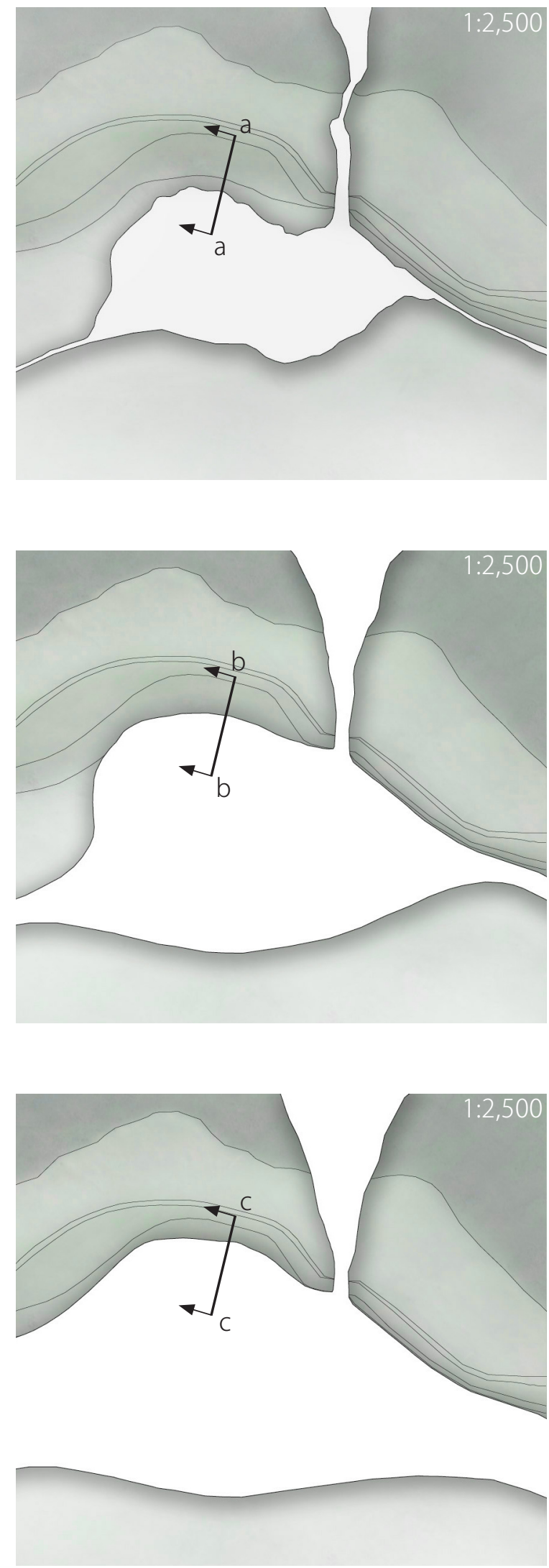

Through the use of a clean water pond, visitors can gain a positive interaction and changing perception, as seen in the Avonhead River project. Water based recreation is encouraged in this space, giving a hands on example of how natural systems can be designed as green infrastructure.

Fig 52.0 Clean water pond section, dry season (Opposite, top)

Fig 53.0 Clean water pond plan, dry season (Above, top)

Fig 54.0 Clean water pond section, wet season (Opposite, middle)

Fig 55.0Clean water pond plan, wet season (Above, middle)

Fig 56.0 Clean water pond section, full flood event (Opposite, bottom)

Fig 57.0 Clean water pond plan, full flood event (Left) 
Lake Wairarapa
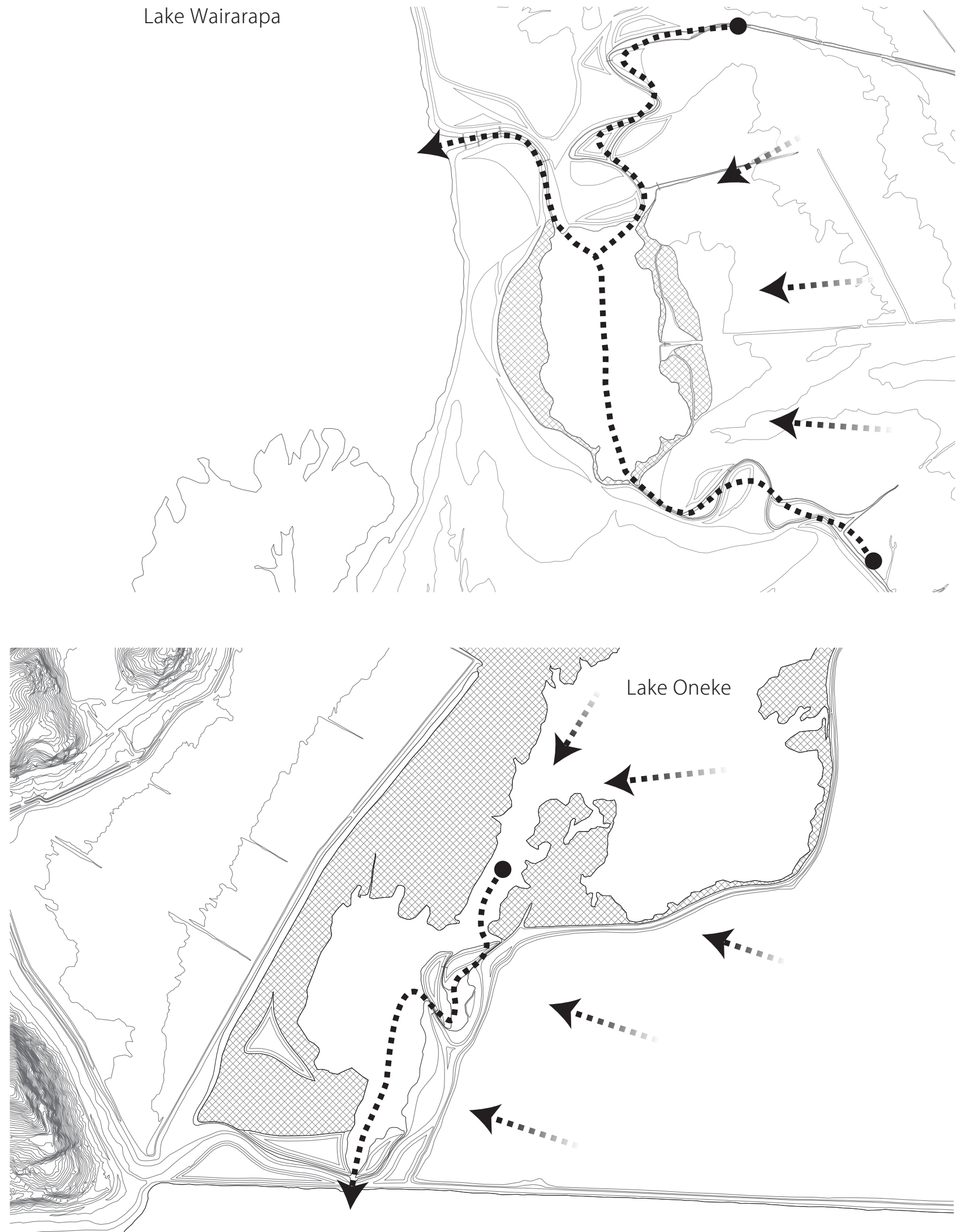
Fig 58.0 Site 1 Diagram (Opposite, top)

Diagram showing the movement of water through the site into Lake Wairarapa and the pollution entering the hydrological system.
The central pond is the end result of surface-flow wetlands and their ability to treat polluted water naturally. Water enters the site using the natural topography of the land, aided by minimal excavation to expose the readily available ground water. The use of 'cut and fill' as a technique to alter the sites ground water has minimal effect on the larger hydrological system. This technique is also the most cost effective approach to wetland restoration on site. Two streams enter from the Northern and Southern ends of the site respectfully, travelling towards the central pond. Upon entering the water runs through a series of wetlands with varying water depths and plant intensities until it reaches the pond as naturally treated, clean water. A natural approach to water treatment provides significant economic benefits to the opposition of chemical treatment, but it also means that the space can be used simultaneously for recreation.

\section{Fig 59.0 Site 3 Diagram (Opposite, bottom)}

Diagram showing the movement of water through the site into Lake Oneke and the pollutants entering the hydrological system. 

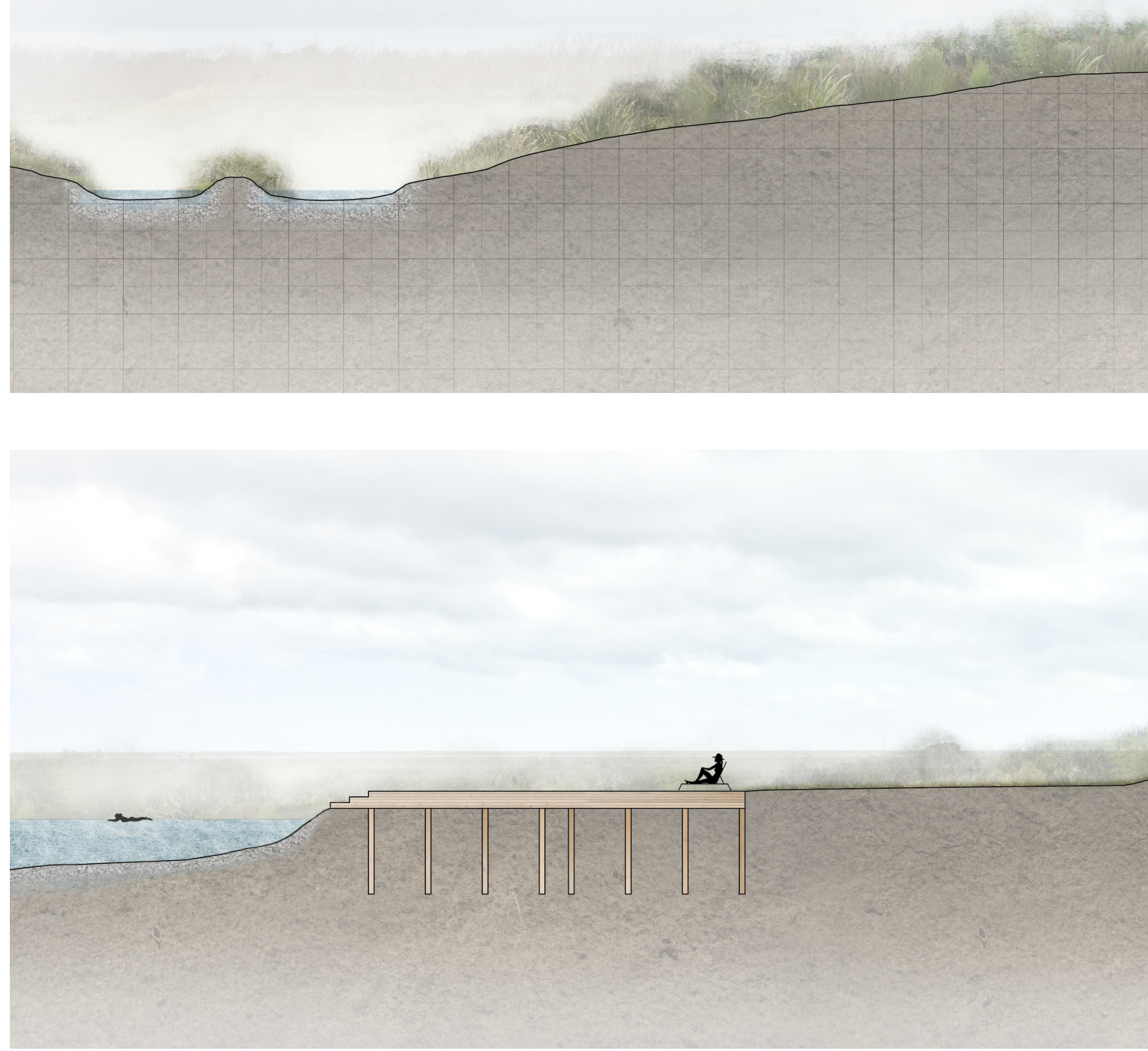

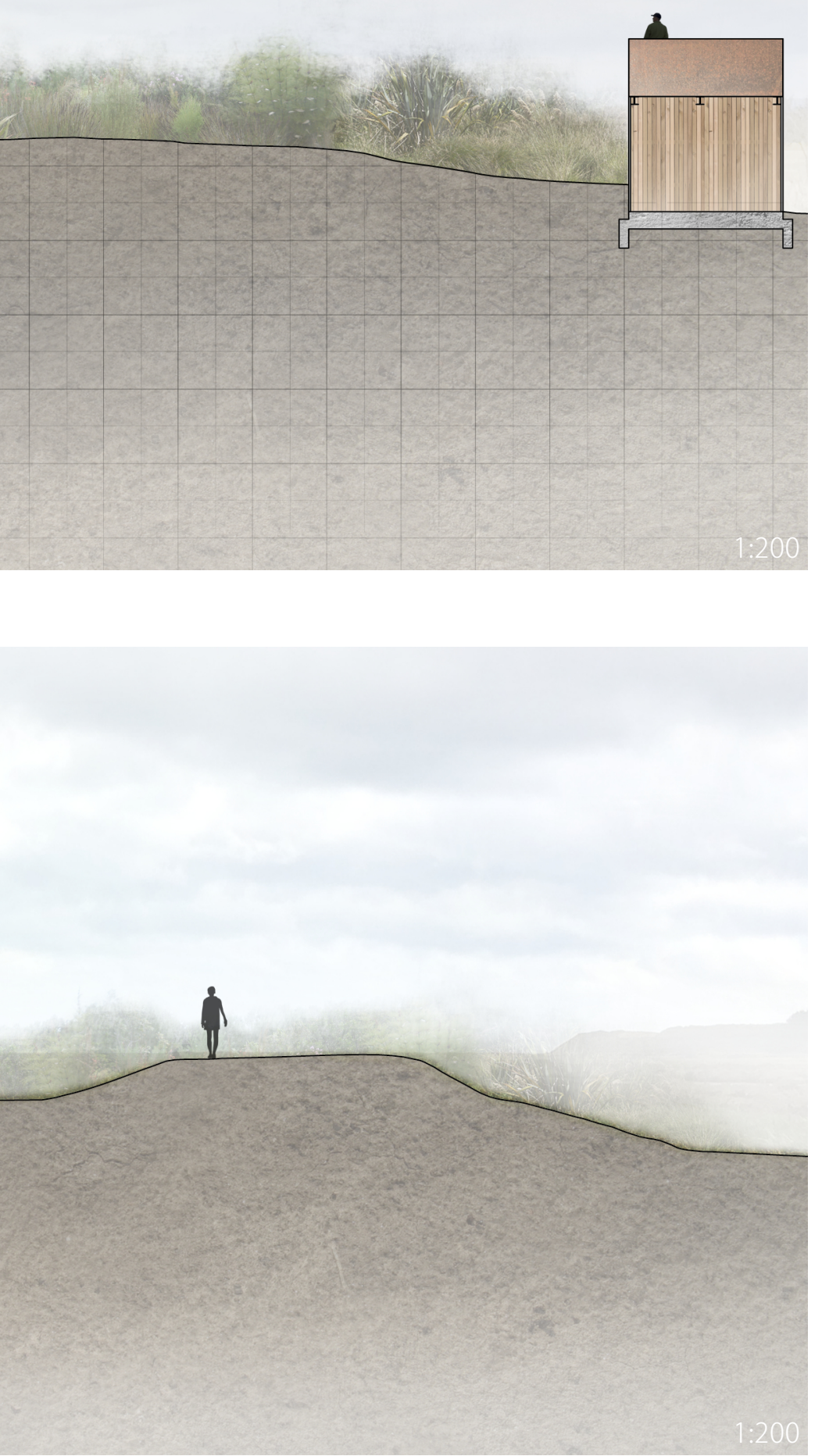

As a fundamental part of the overall design that has a powerful effect at many scales, the hydrology has been designed in unison with recreation and human experience. By allowing people to view the water treatment as it is happening, and then use the clean water at the end of the process, a positive relationship is formed. This site's hydrology will act as a catalyst for further design around the region in an effort to restore the water quality of Lake Wairarapa to a former functioning level. The economic feasibility of the intervention also means the time line for design implementation is achievable as a long term goal for the region. Starting first at site two and then spreading outward to sites one and three respectfully.

Fig 60.0 Site 1 typical section

Section cut showing the placement of view platform looking over newly constructed wetland water treatment streams.

Fig 61.0 Site 3 typical section

Section cut showing the placement of the constructed deck and steps overlooking the clean water pond for recreational use. 


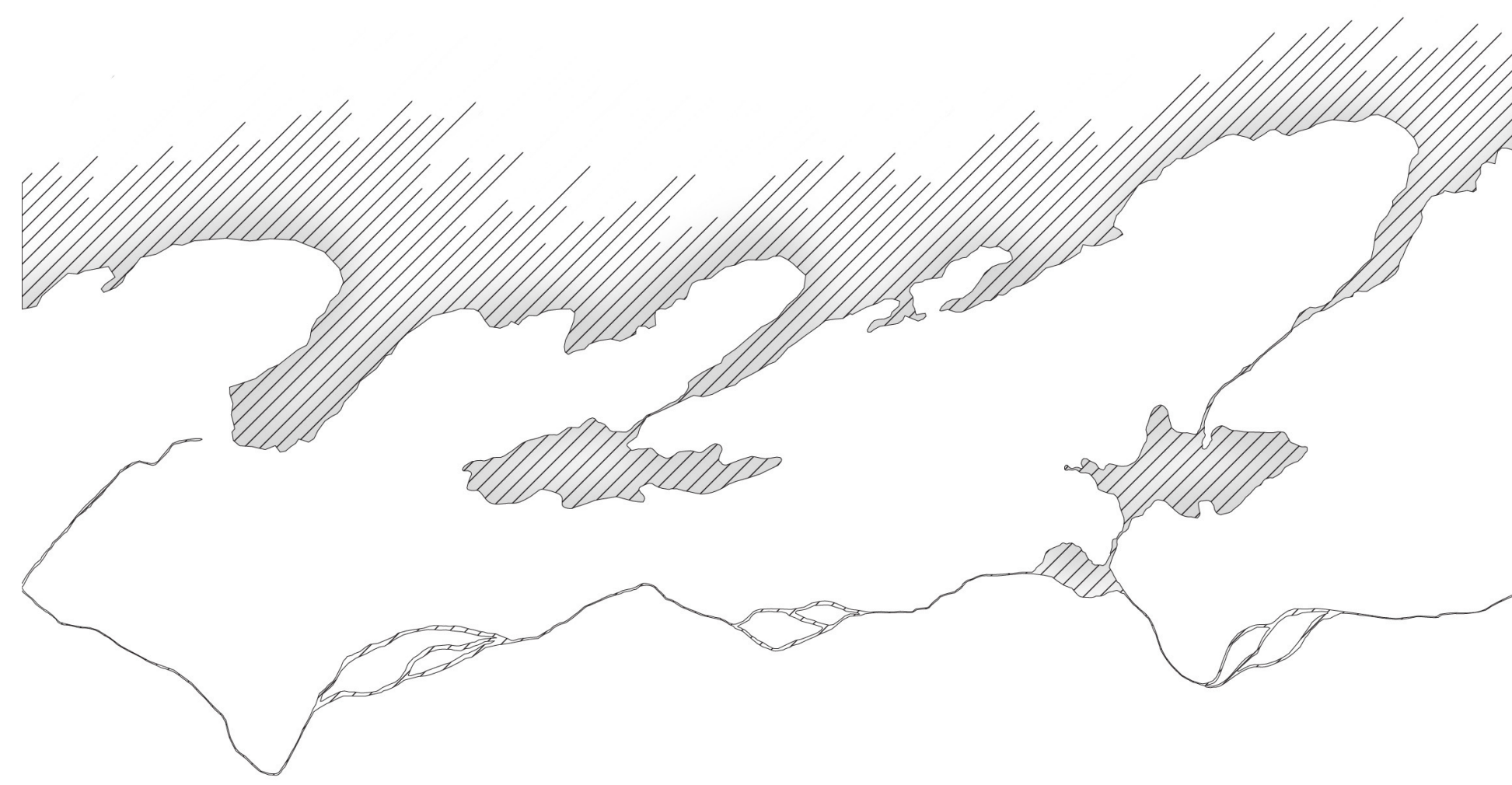




\subsubsection{DESIGNING FOR FLUX}

Landscapes are dynamic systems embedded in a flux of energy, materials, nutrients and species. Disturbing this flux has a massive impact in several natural cycles such as the hydrologic. The flux generated from water tends to be overlooked or artificially controlled, impacting the function of our landscapes, which can lead into flood sediment build up and ultimately ecosystem destruction. Lake Wairarapa is a prime example of natural flux being removed.

Flood events are historically viewed as a problem in Wairarapa Moana, rising water levels drown the farmlands making them unproductive for long periods of the year. Artificial stop banks have been implemented to ensure this flux is controlled and removed from the land ensuring the farmland is as productive as possible. This design encourages the flux of the lake to enter the system, harnessing the water it brings with it. During the wet seasons of the year, the designed wetlands naturally expand to take on more water. (Fig 63.0) Acting like a sponge, the surface flow wetlands

Fig 62.0 Site 2 water level, standard (Above) are capable of taking on and treating a 


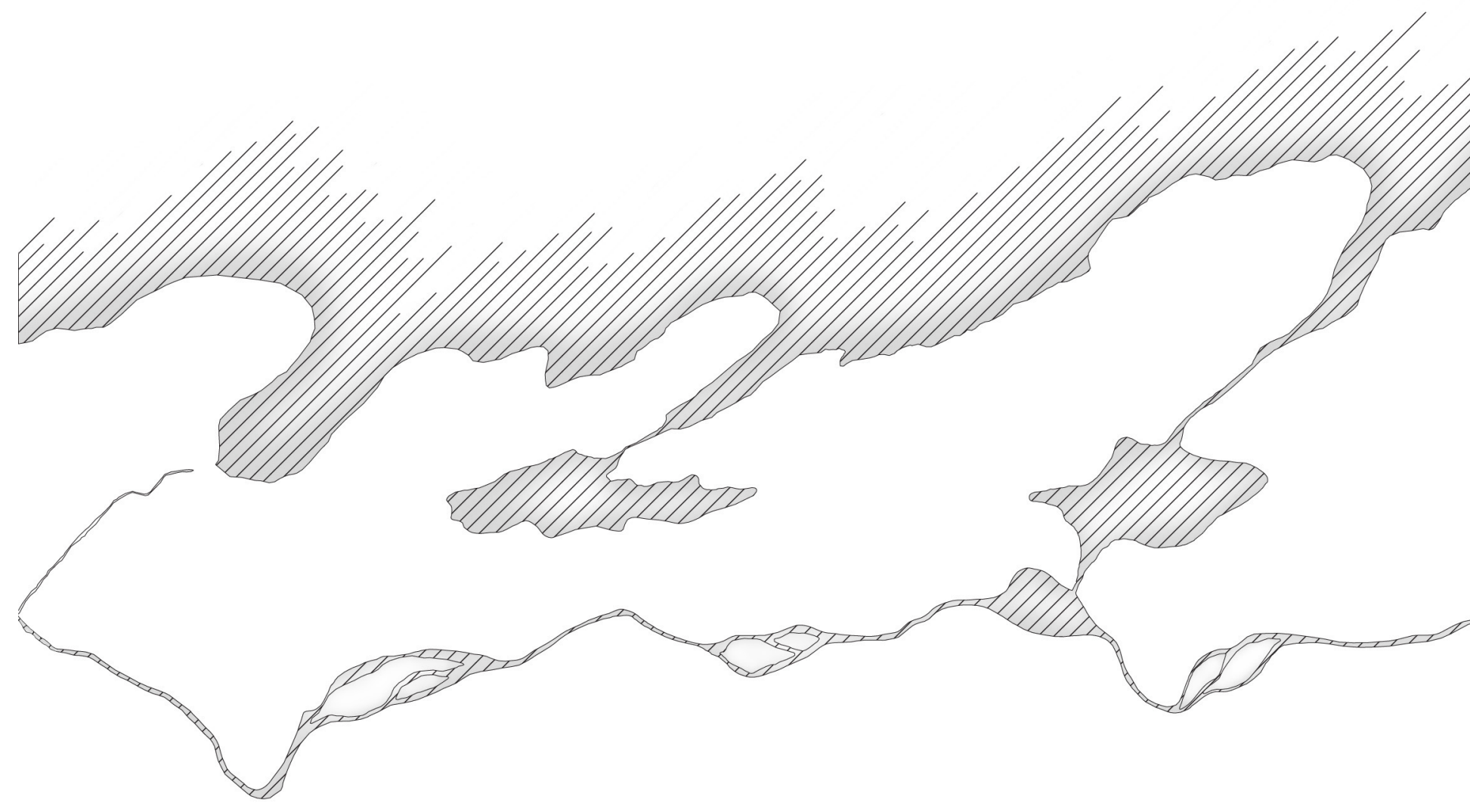




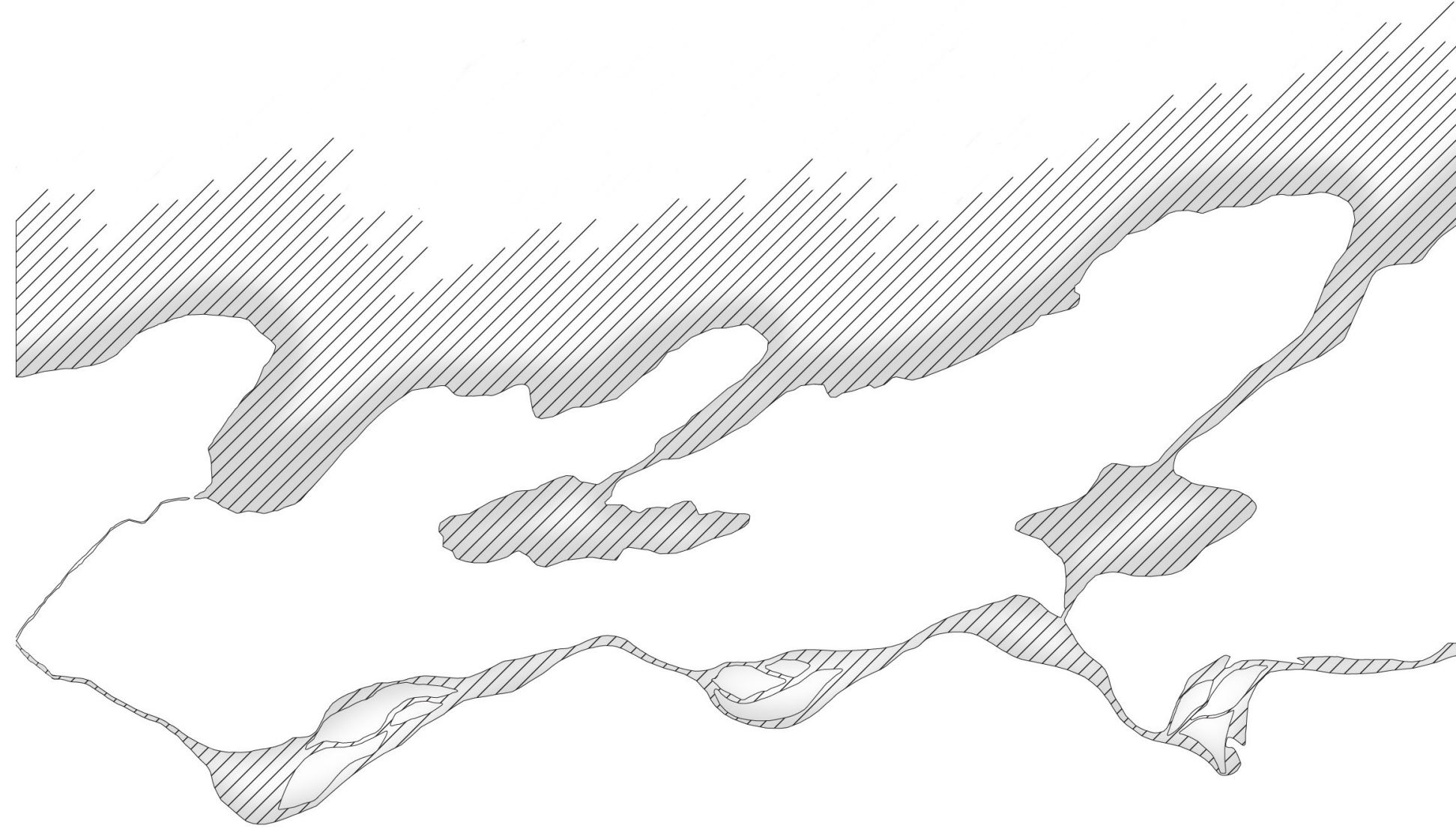



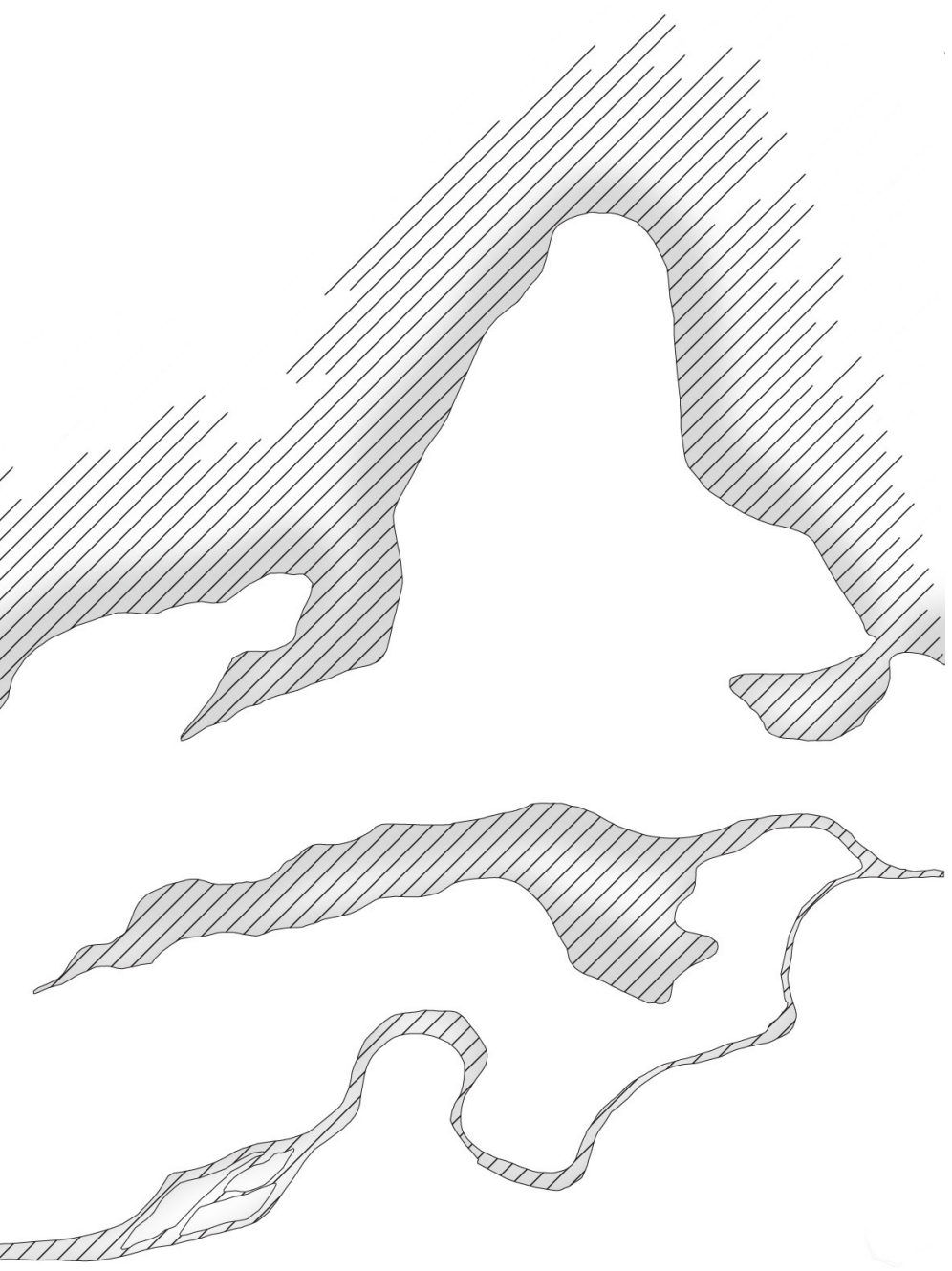

Fig 64.0 Site 2 water level, maximum

(Above) of a traditional approach, any artificial intervention must be designed as naturally occurring process. Each site around Wairarapa Moana is different, an understanding of site specific processes is vital in the success of designing with flux in the landscape. (Fig 64.0-67.0) 


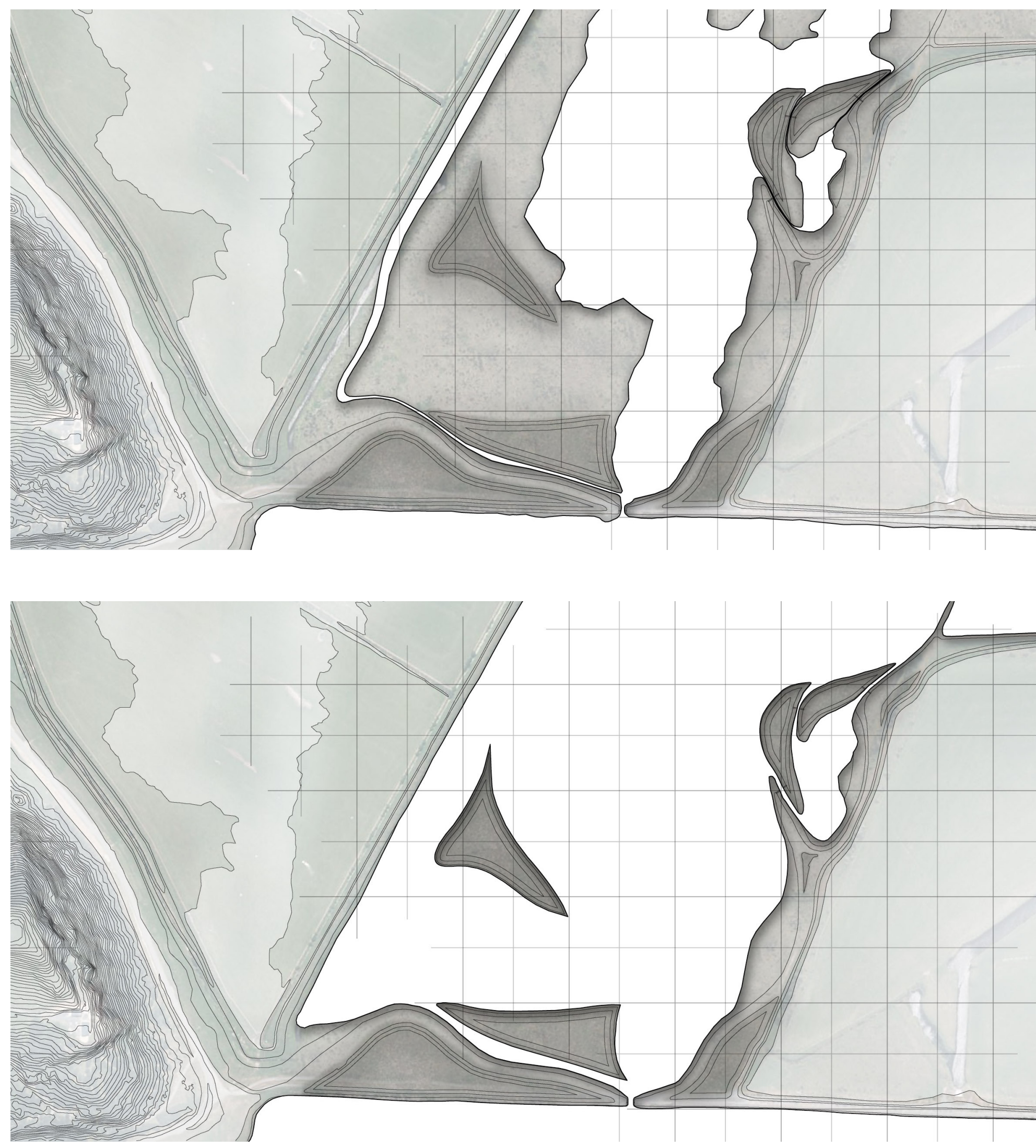

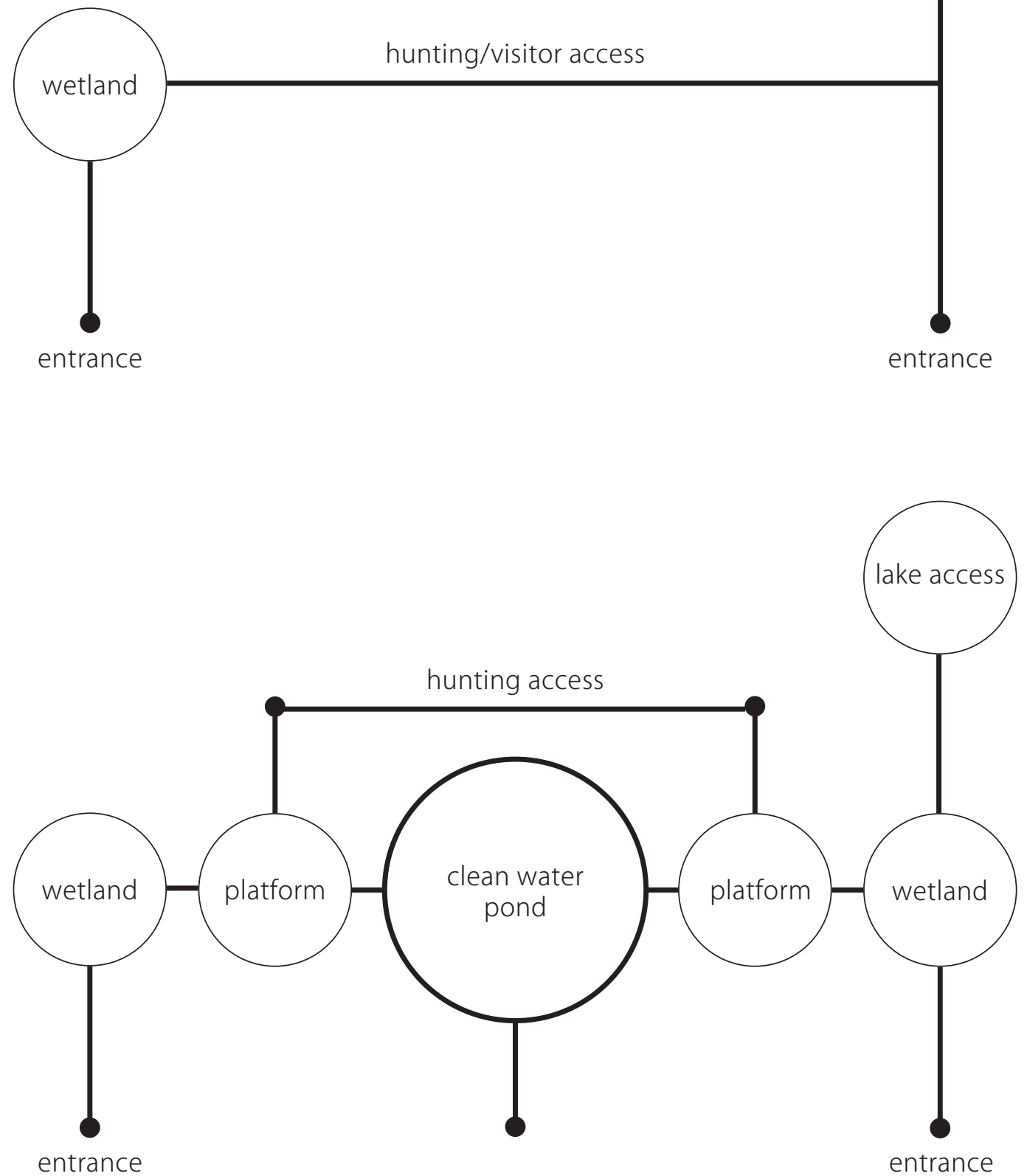


\subsubsection{ECONOMICAL LANDSCAPES}

The South Wairarapa has a very low population to land area ratio, therefore economically feasible design is crucial. Infrastructure heavy solutions typically alter natural systems having a detrimental effect on the native function of the landscape, but also have a significant cost attached. The implementation of green infrastructure works in parallel with the native function of the landscape and provides a much more economical feasible solution when limited funding is available. The economic viability of a design has significant implications, the success of this research as economically feasible for the South Wairarapa means it could act as a new standard of regenerative design for the region. Landscape architecture can be used to lessen the adverse effects of poor land use and management when limited financial assistance is available, through the use of natural systems.

A typical approach to a conservation area results in very basic infrastructure such as boardwalks and navigational aids. Through the vessel of landscape architecture, the typical Department of
Conservation (DOC) can be pushed to createauniquespacethatgives Wairarapa Moana a unique identity. Visitors to the site require very basic amenities such as access ways and formed walking tracks, large interventions in this landscape would not fit with the character of the landscape and would be unaffordable (Fig 69.0). By using basic structures, materials and moulding the existing site a much more realistic and economically feasible design is created. The most dominant interventions of this design, the view platforms, are bold enough to suggest intervention, yet subtle enough in the execution of design and materiality to be affordable. The manipulation of the existing stop banks is also a design feature that has come at an affordable cost. (Fig 72.0)

\section{Fig 69.0 Site 2 current access diagram (Top)}

Limited circulation around the site is currently available for visitors to site.

Fig 70.0 Site 2 proposed access diagram (Bottom)

Proposed circulation around the site offering multiple routes and activities. 


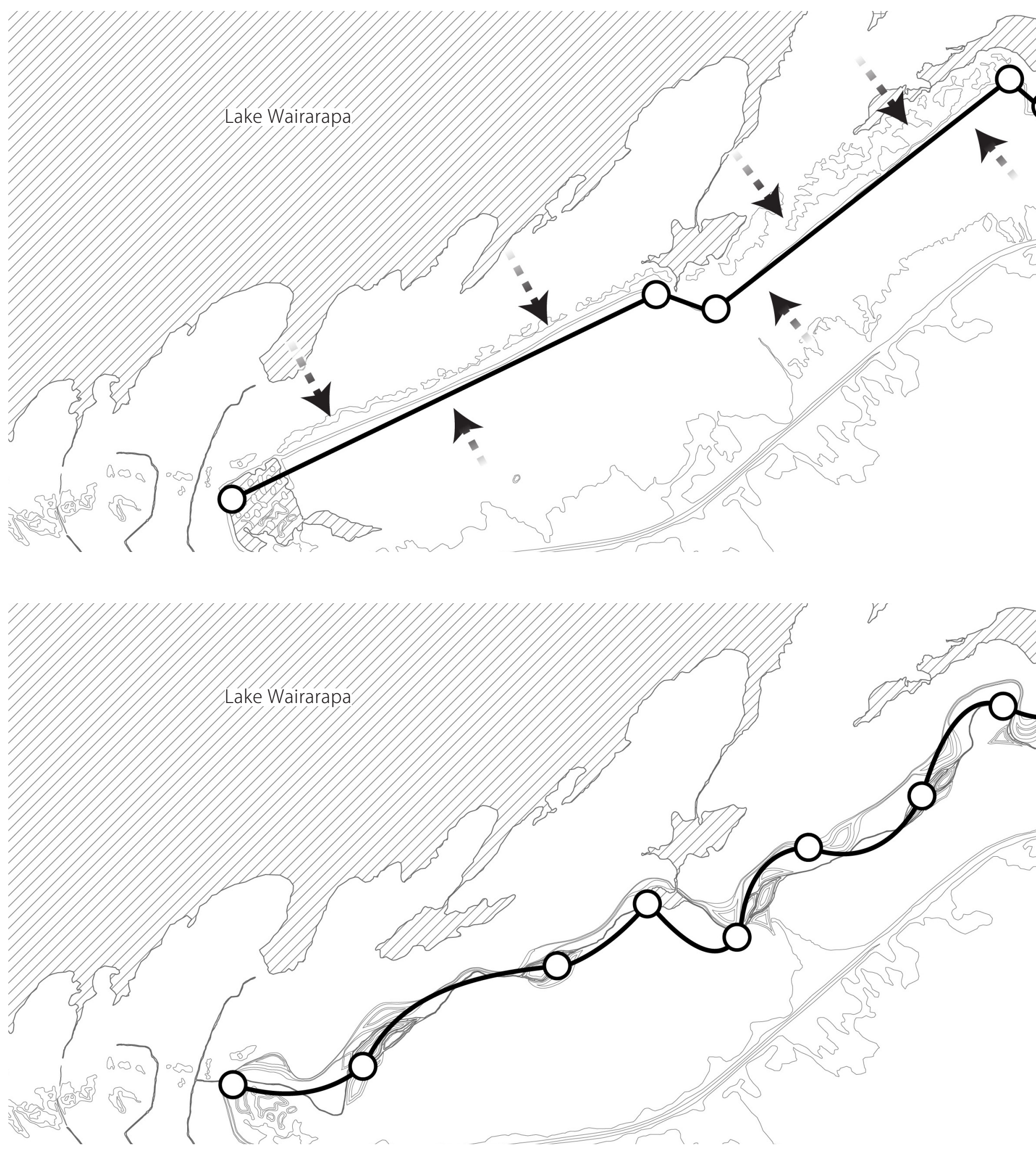



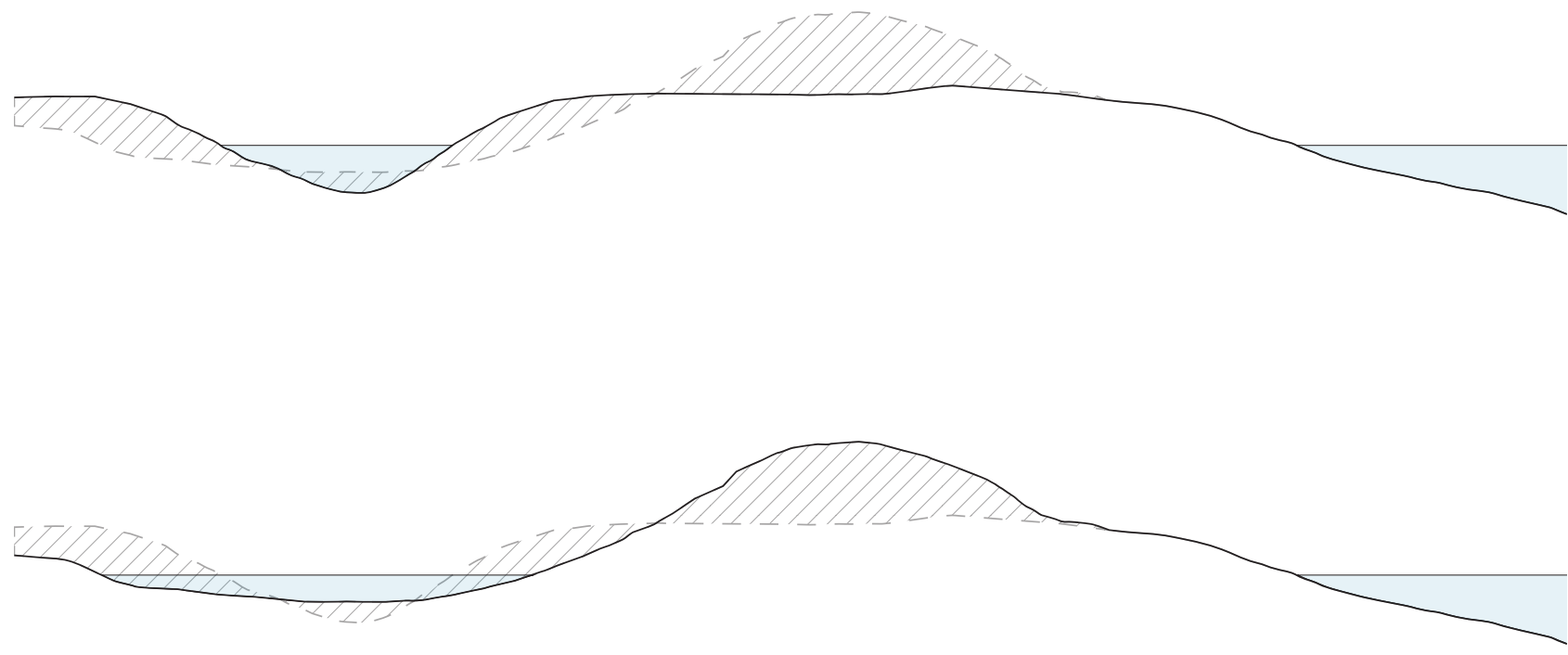

Fig 73.0 Site 1 cut and fill diagram
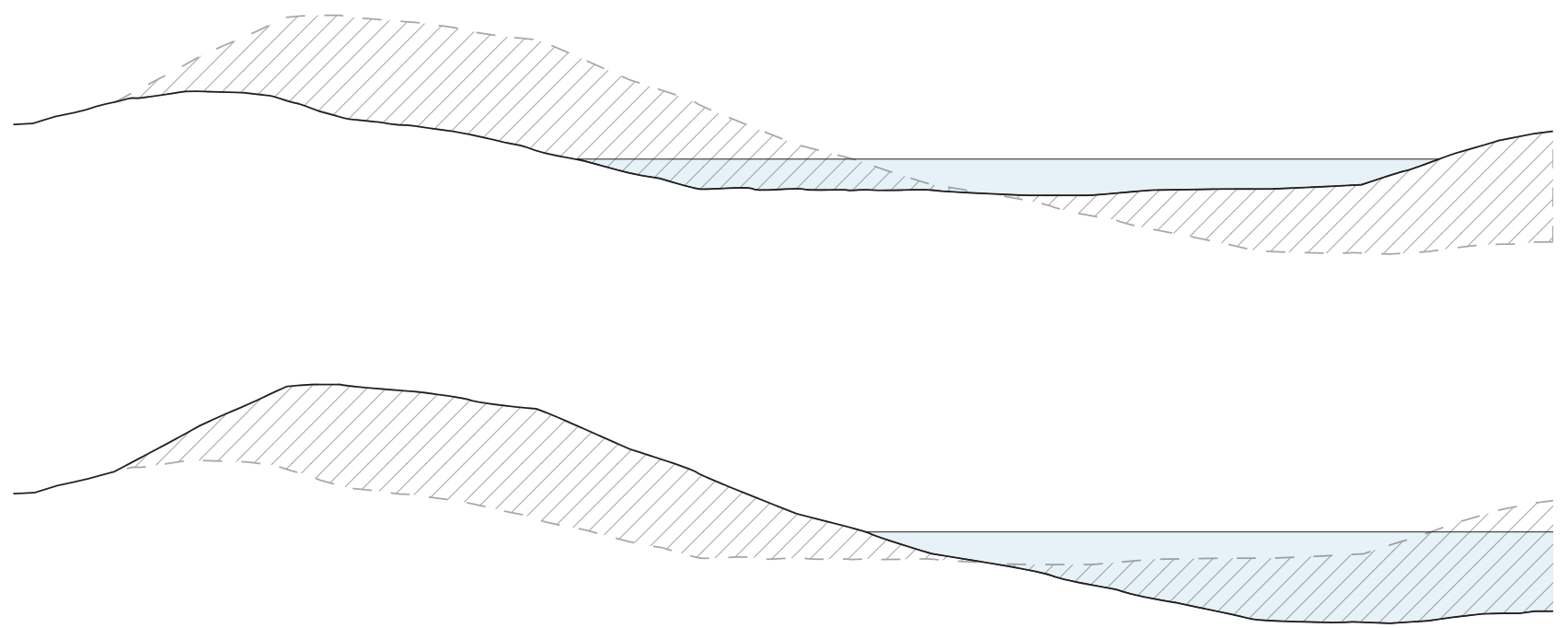

Fig 74.0 Site 2 cut and fill diagram 

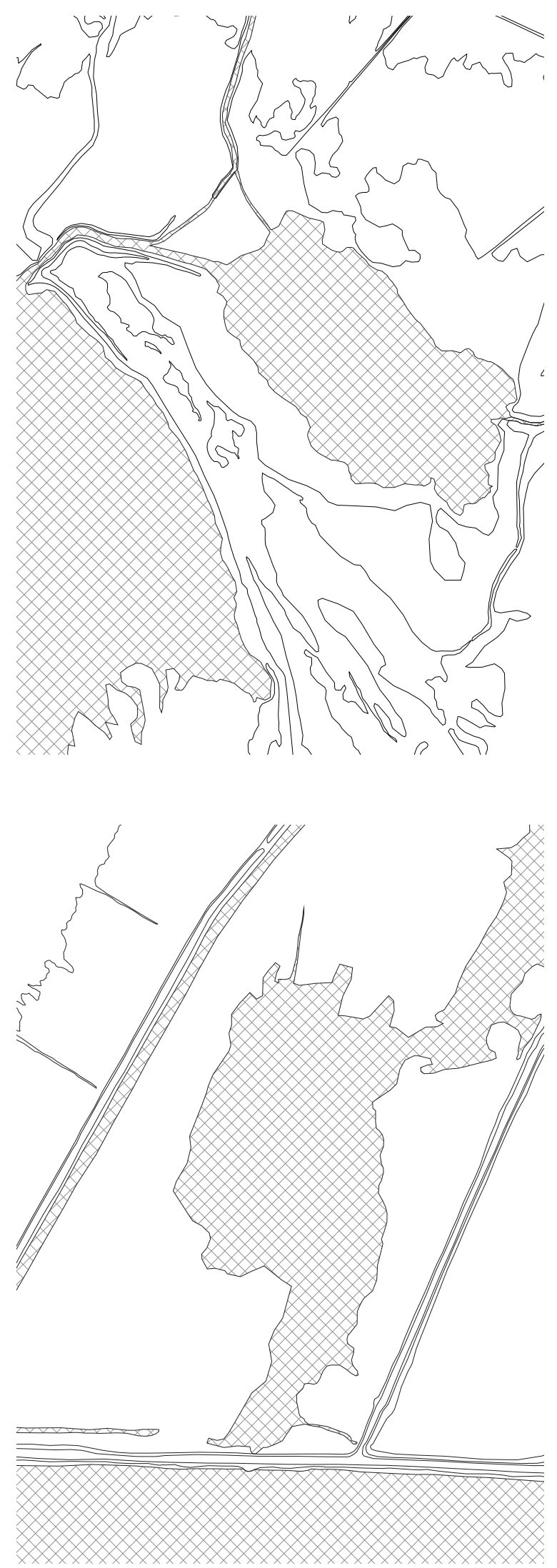

The use of cut and fill to create a unique landscape experience that still fits with the character of the South Waiarapa Landscape is imperative in the success of this design. Through using a palette of materials that already exists on site restraint is shown to add to the experience of the site, while still remaining financially feasible for the region. The technique of cut and fill to create height in such an expansive flat landscape has proven to be very successful in varying the horizontal plane. (Fig 73.0) (Fig 74.0) The financial feasibility of this design was crucial for its success, a challenge that has been met and exceeded to create a unique space to draw visitors to Wairarapa Moana and encourage a positive relationship with the surrounding land.
Fig 75.0 Site 1 plan reference (Top)

Fig 76.0 Site 3 plan reference (Bottom) 


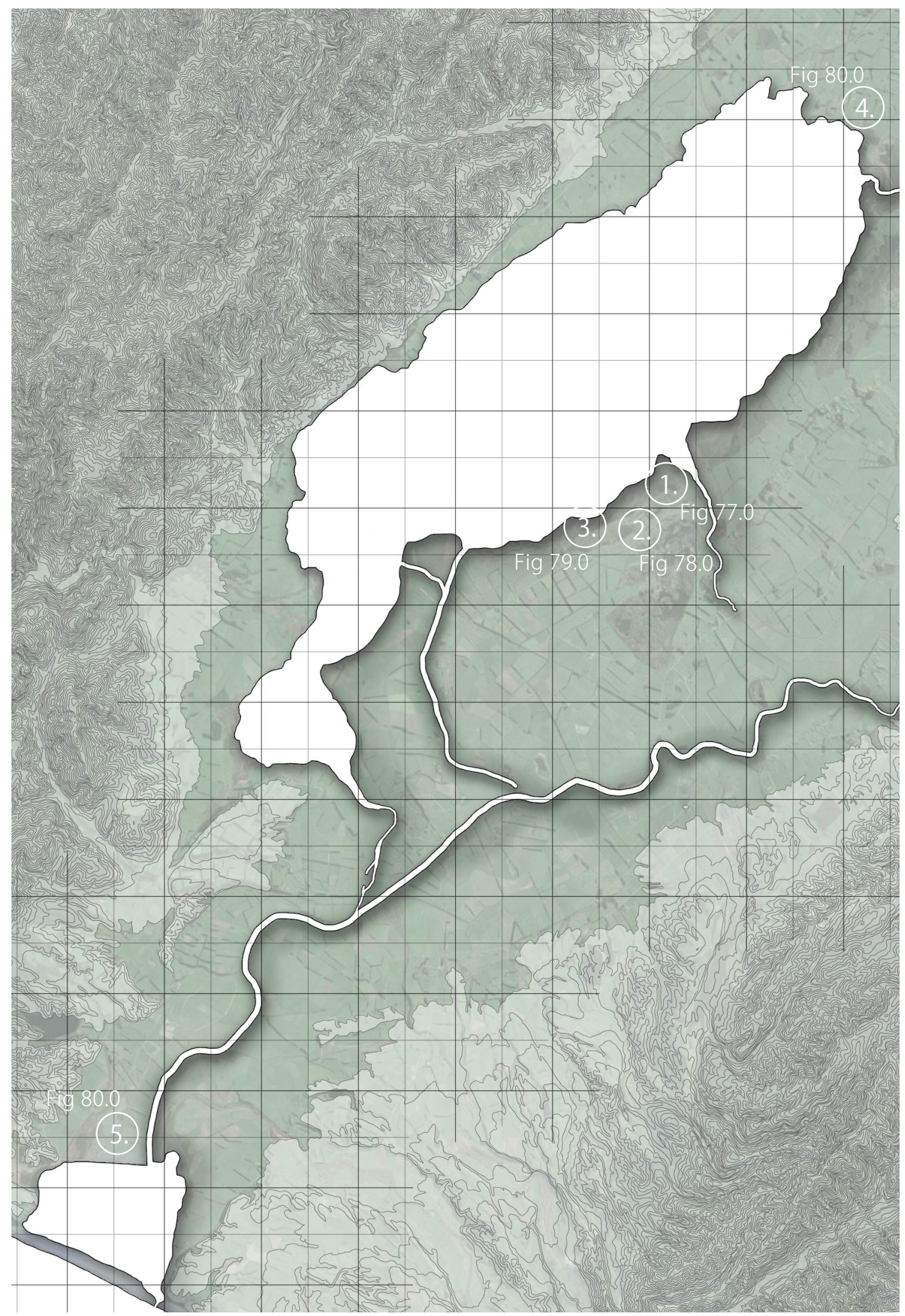




\subsubsection{RECREATIONAL INFRASTRUCTURE}

Exceeding the limits of a typical landscape architecture approach to a site requires a much more elaborate process of design. Infrastructure is used as a solution to a problem within the landscape, often landscape architecture is used as a vessel to mitigate the effects of such infrastructure on natural systems. The wetland system designed for Wairarapa Moana is an integration of infrastructure with designed space, the process for design evolved as a synergy rather than two elements working side by side. The designed open space allows visitors to interact with such infrastructure at a personal scale, features of the design have a dual function of human experience and function. The new typology of landscape architecture, referred to as recreational infrastructure, meets the needs of humans and the landscape as one system.

Fig 77.0 Regional perspective reference plan (Opposite) 


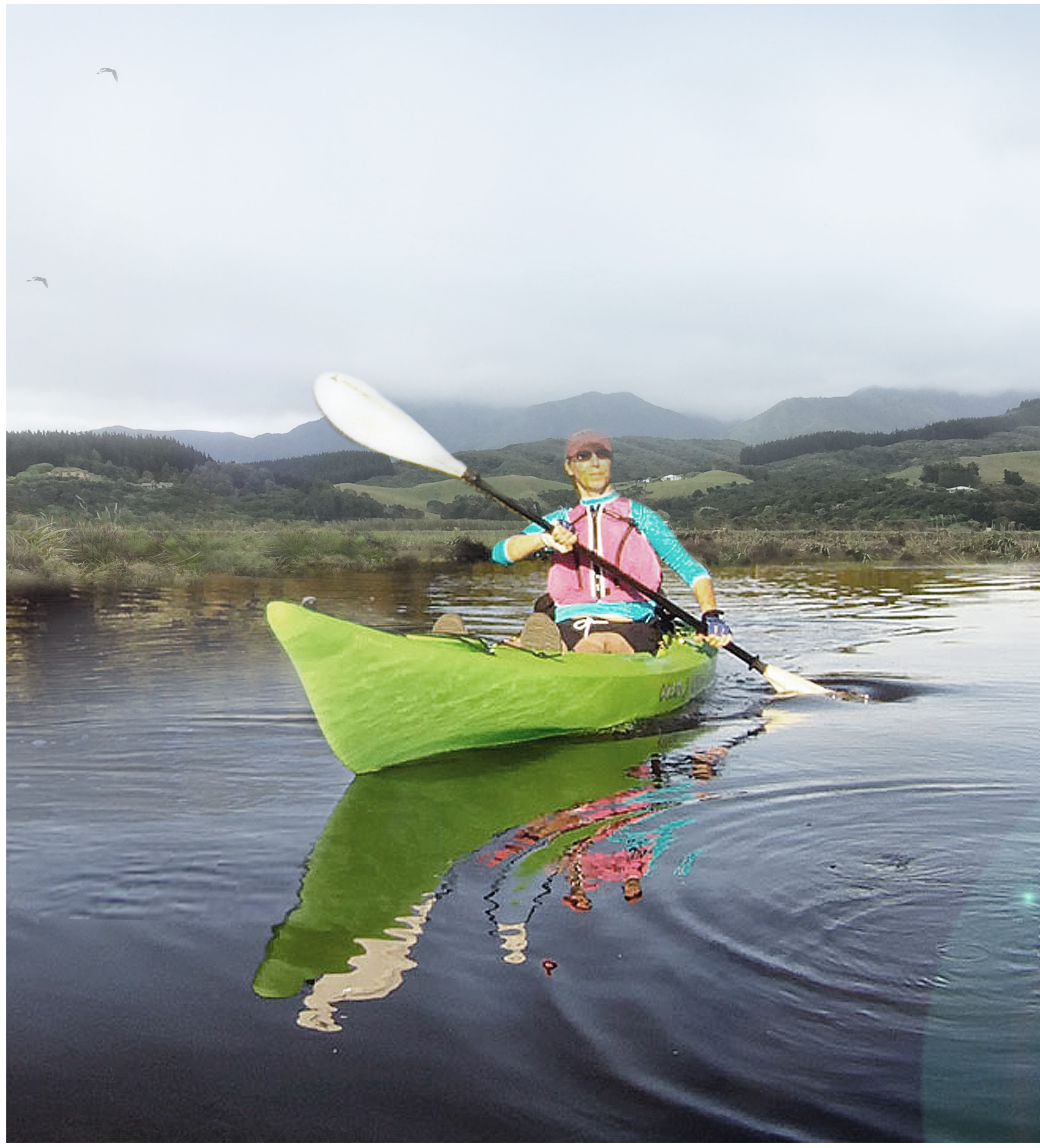




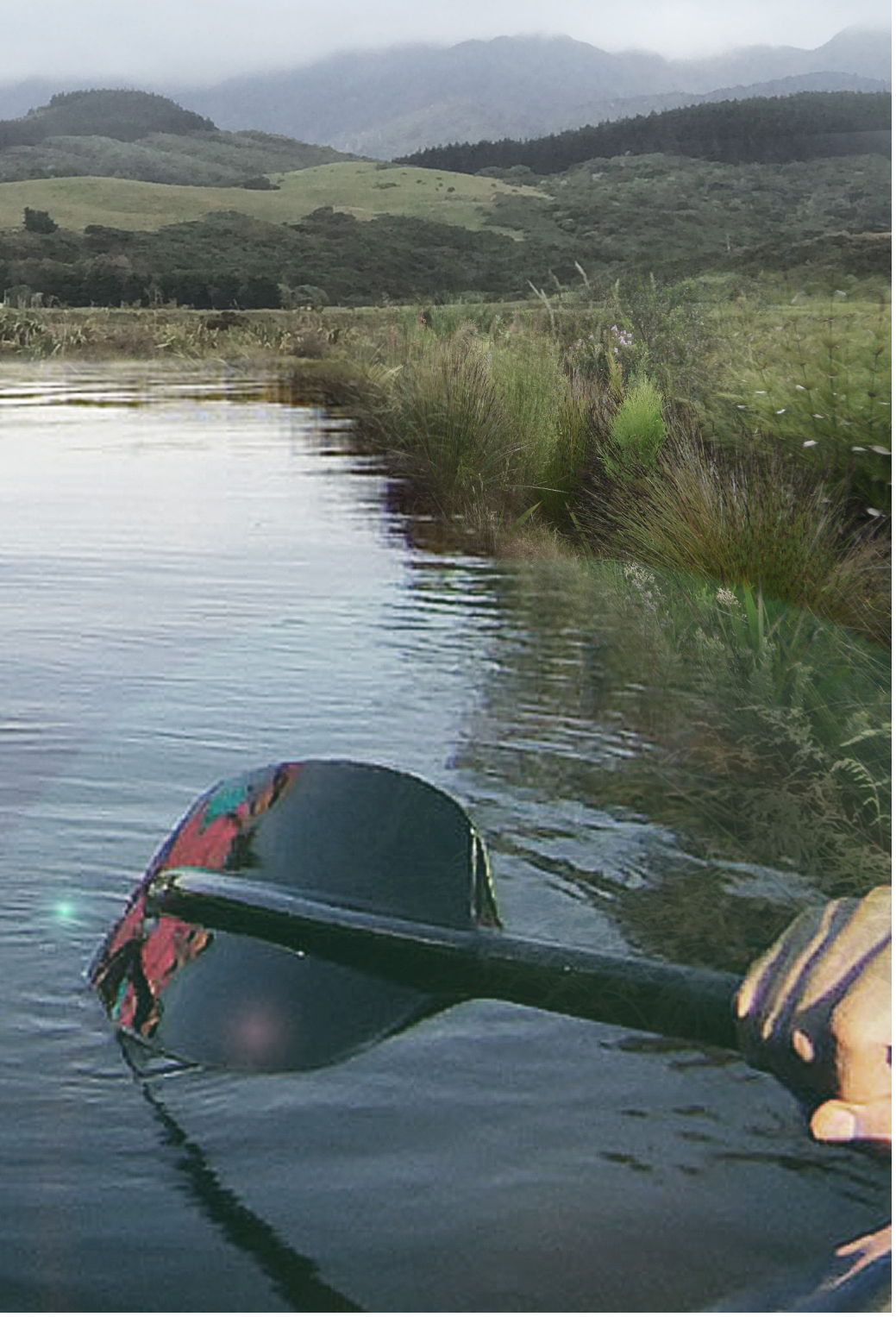

The function of the landscape however can be split into two for clarity of understanding: the needs of the natural system and the needs of the human users. The needs of the natural system can be generalised to water treatment, a natural approach to this problem should be taken into account. A solution that works for the landscape, not against. Modelled on the wetlands that once covered this site, the function of the space is to remove pollutants and return clean water back into the lakes hydrological system. (Fig 78.0) The reintroduction of wetlands are a natural solution to water pollution issues. (Fig 83.0)

Fig 78.0 Site 2 recreational Lake (Opposite) Refer to page 124, (1) 


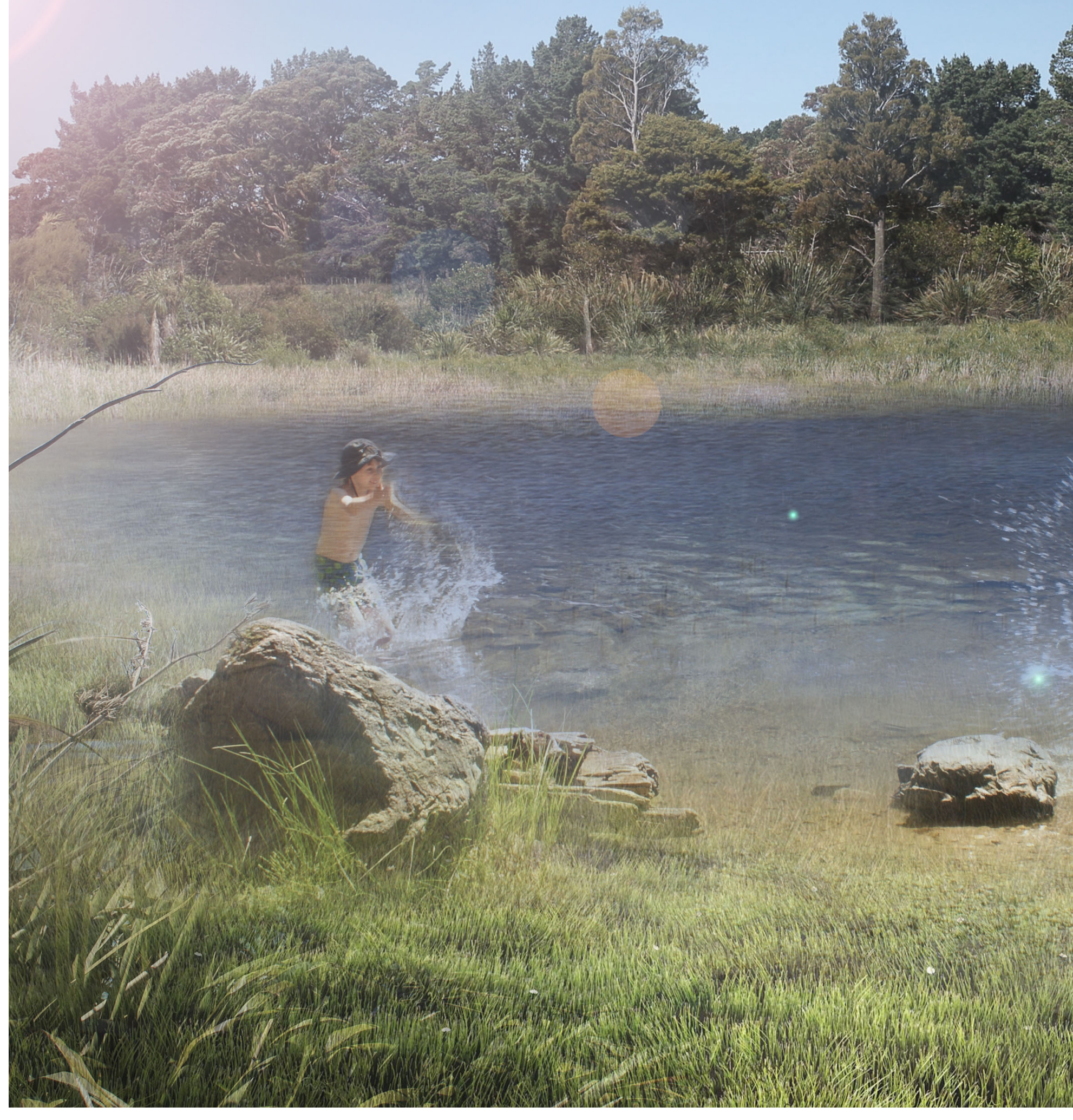




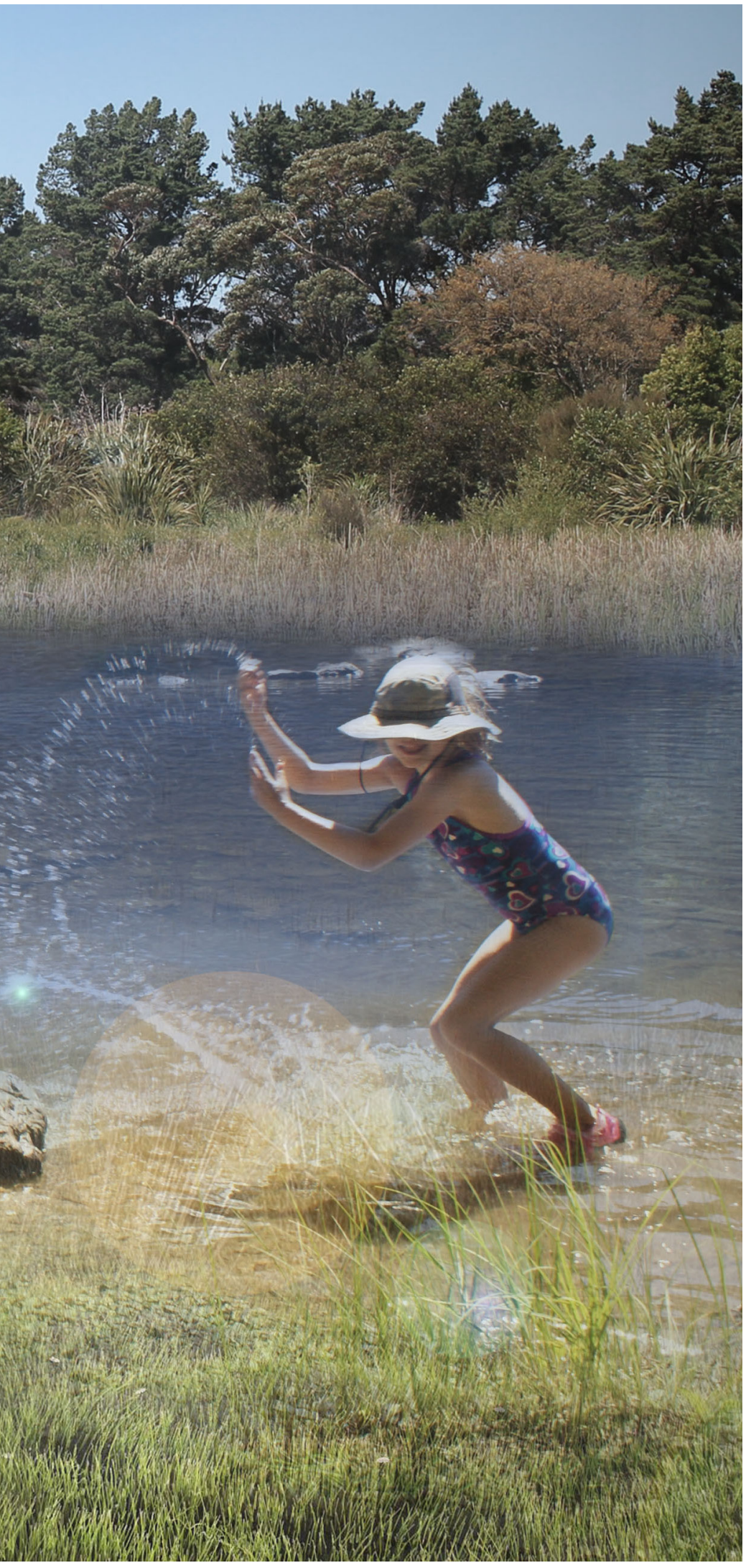

The function of the landscape for human users requires more rigor and consideration to meet the varying needs of visitors. A dynamic landscape that affords multiuse space is the result of these needs being met. Circulation around the site is depended on the visitor; a personal experience is created through a journey created by the individual. (Fig 79.0) A large variation in path lengths, multiple destinations and freedom of navigation allows the site to cater to all. (Fig 88.0 - 89.0)

Fig 79.0 Site 2 clean water pond (Opposite) Refer to page 124, (2) 


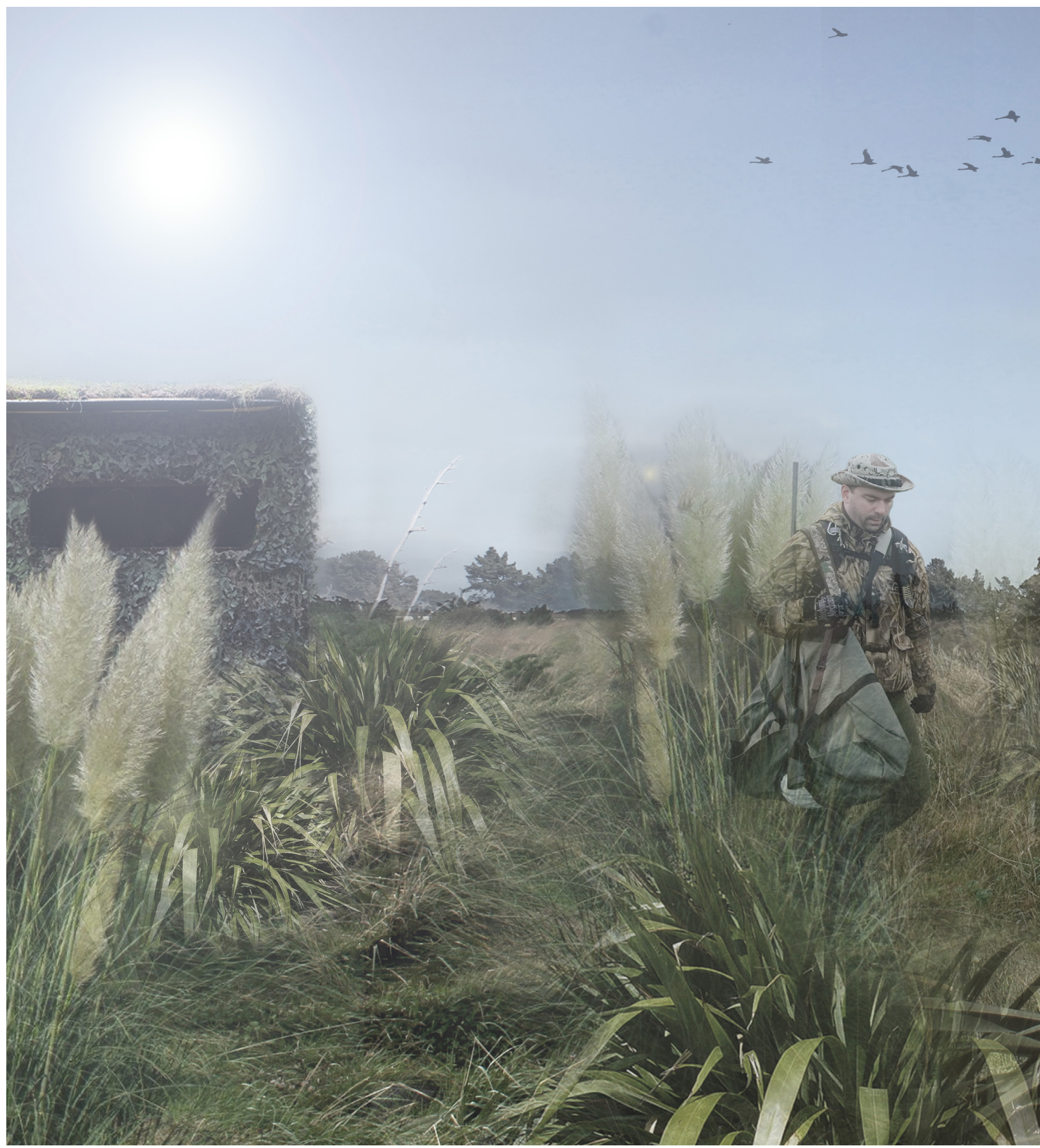




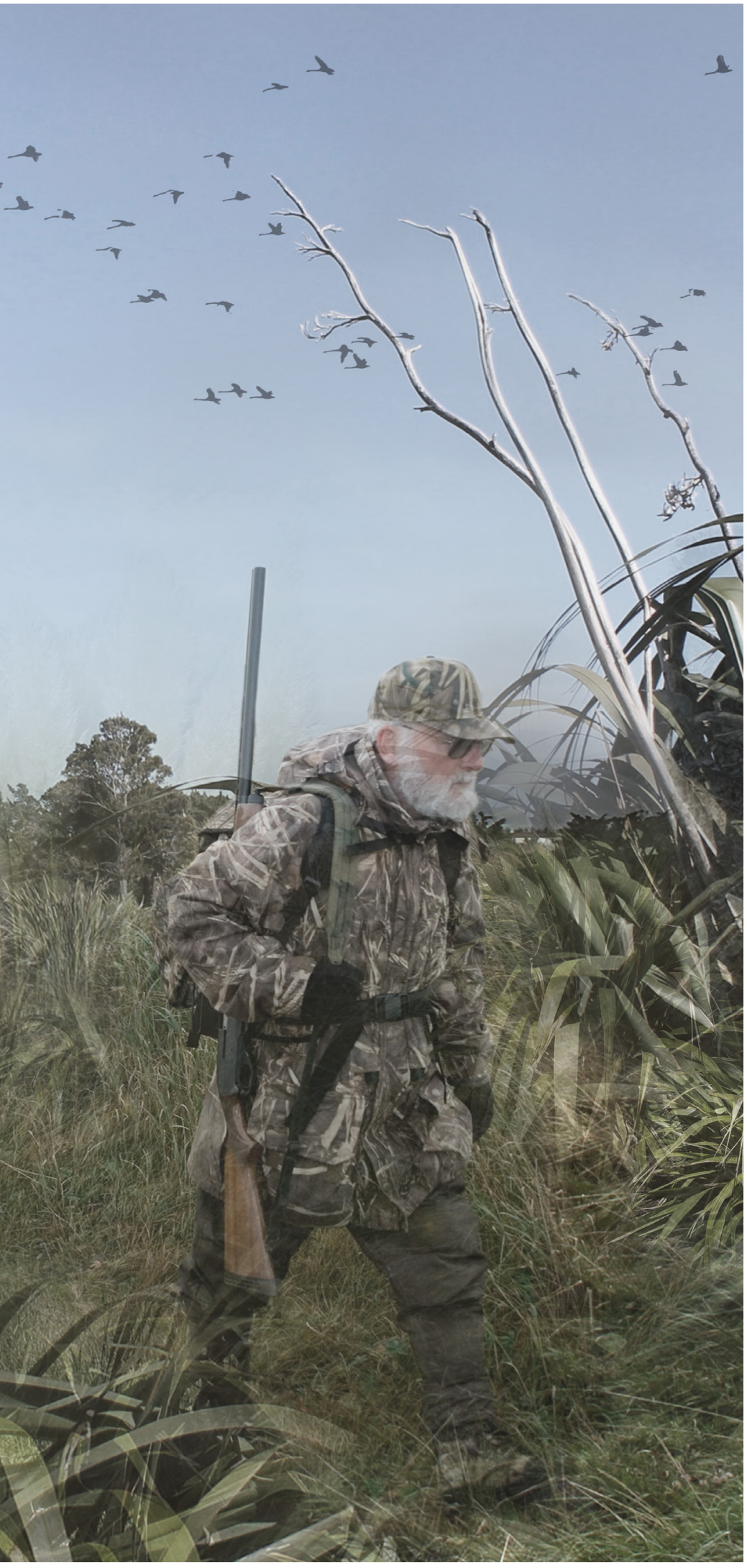

Recreation on site is supported by the facilitation of multiple programmes. Swimming in the central water body creates a space for all ages to relax and learn the importance of water safety. Access to larger lakes support kayaking, duck hunting and bird watching are readily available throughout the site. (Fig 80.0) View platforms allow the more relaxed visitor to enjoy a new view on Wairarapa Moana. The introduction of height through elevated viewing platforms allows this to happen.

Fig 80.0 Site 2 hunting shelter (Opposite) Refer to page $124,(3)$ 


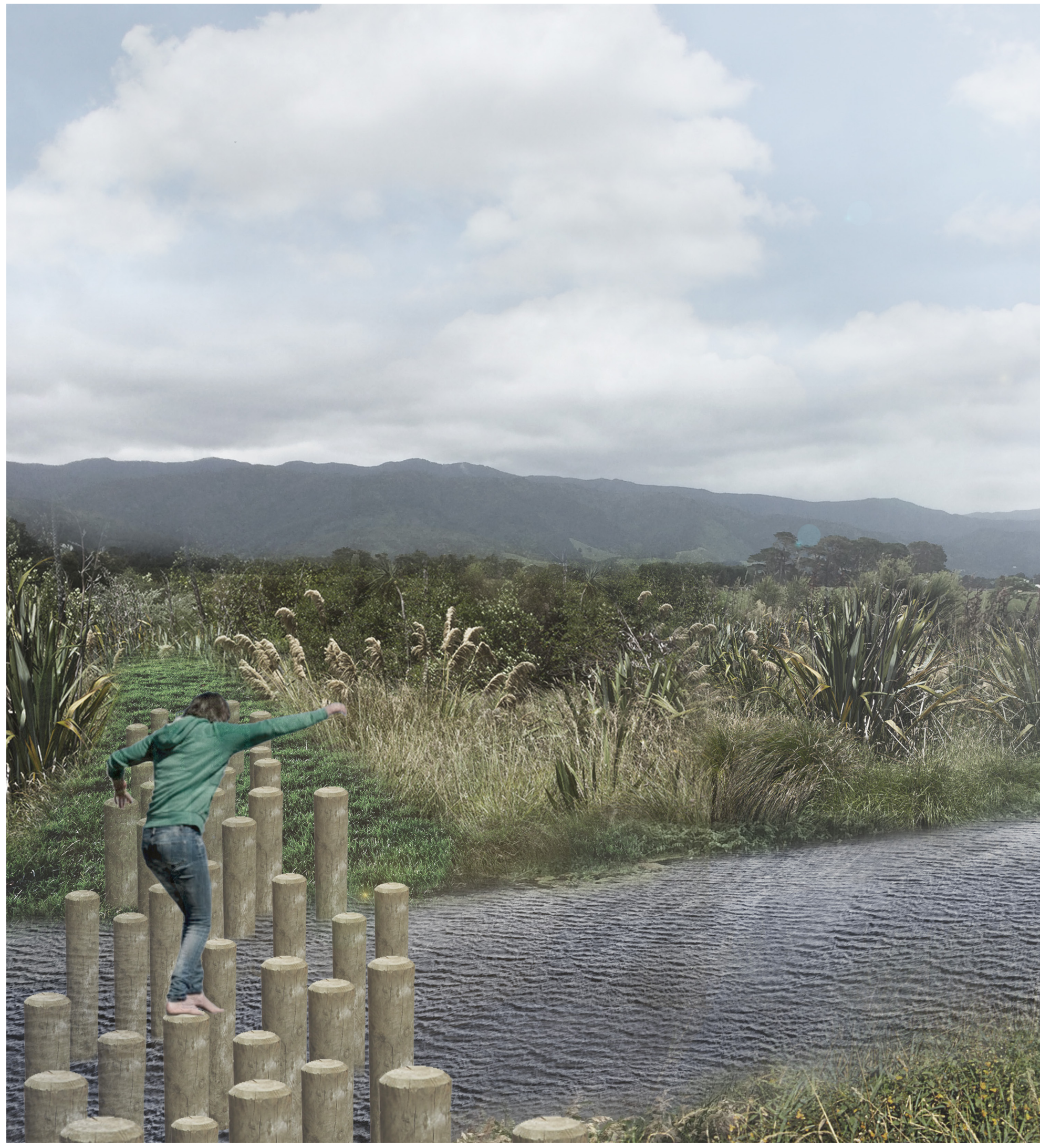


Water play integrated within these platforms also provides a number of useful facilities. The ability to use treated water for cleaning is provisioned also as the more adventurous users will discover damp soil features heavily around the lake shore. A chance for adults to join the young engaging in some nostalgic play with dirt and mud. (Fig 81.0) Designing for people and the landscape simultaneously achieves a diverse space that raises the disciplinary tendency for regional design within New Zealand.

Fig 81.0 Site 1 water crossing (opposite) Refer to page 124, (4) 


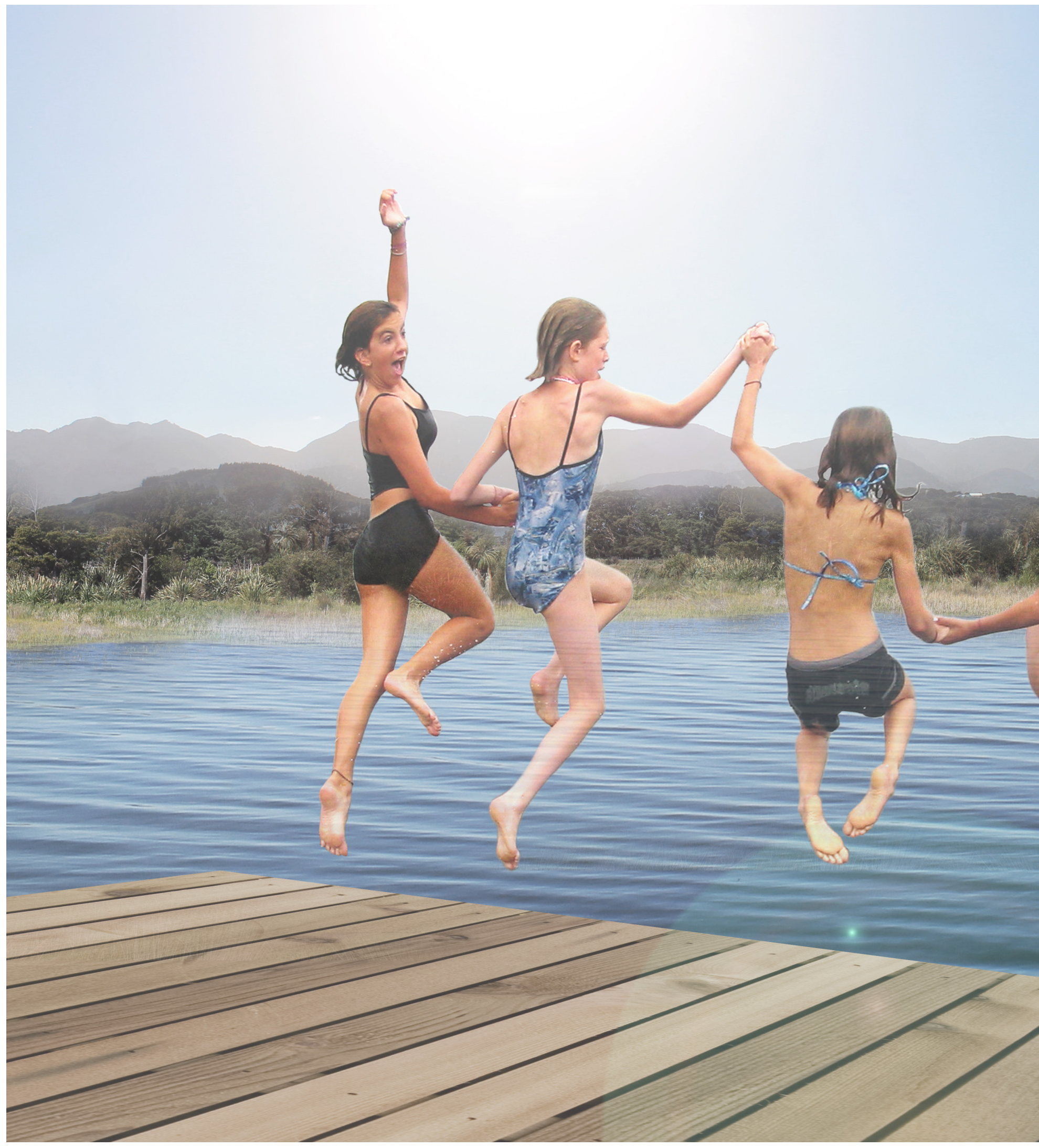

122 
Applied at a regional scale, a varying range of water based interventions begin to evoke the thought of play for visitors. As water is the feature in all sites around the region, each design must be responsive to this aspect. Responsive in such a way that a positive relationship with the much needed infrastructure is gained from experiencing the designed landscape.

Fig 82.0 Site 3 water deck (Opposite)

Refer to page 124, (5) 


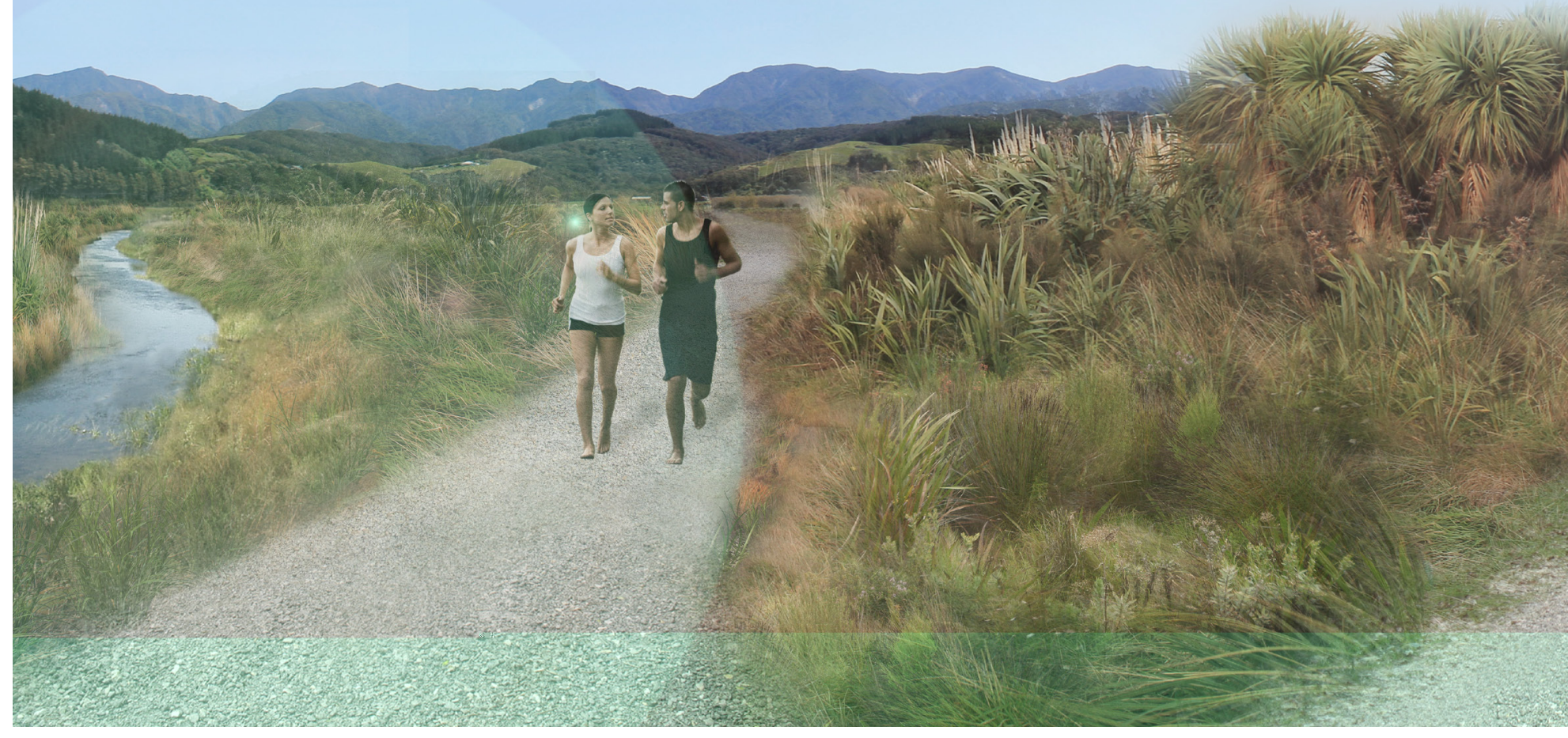




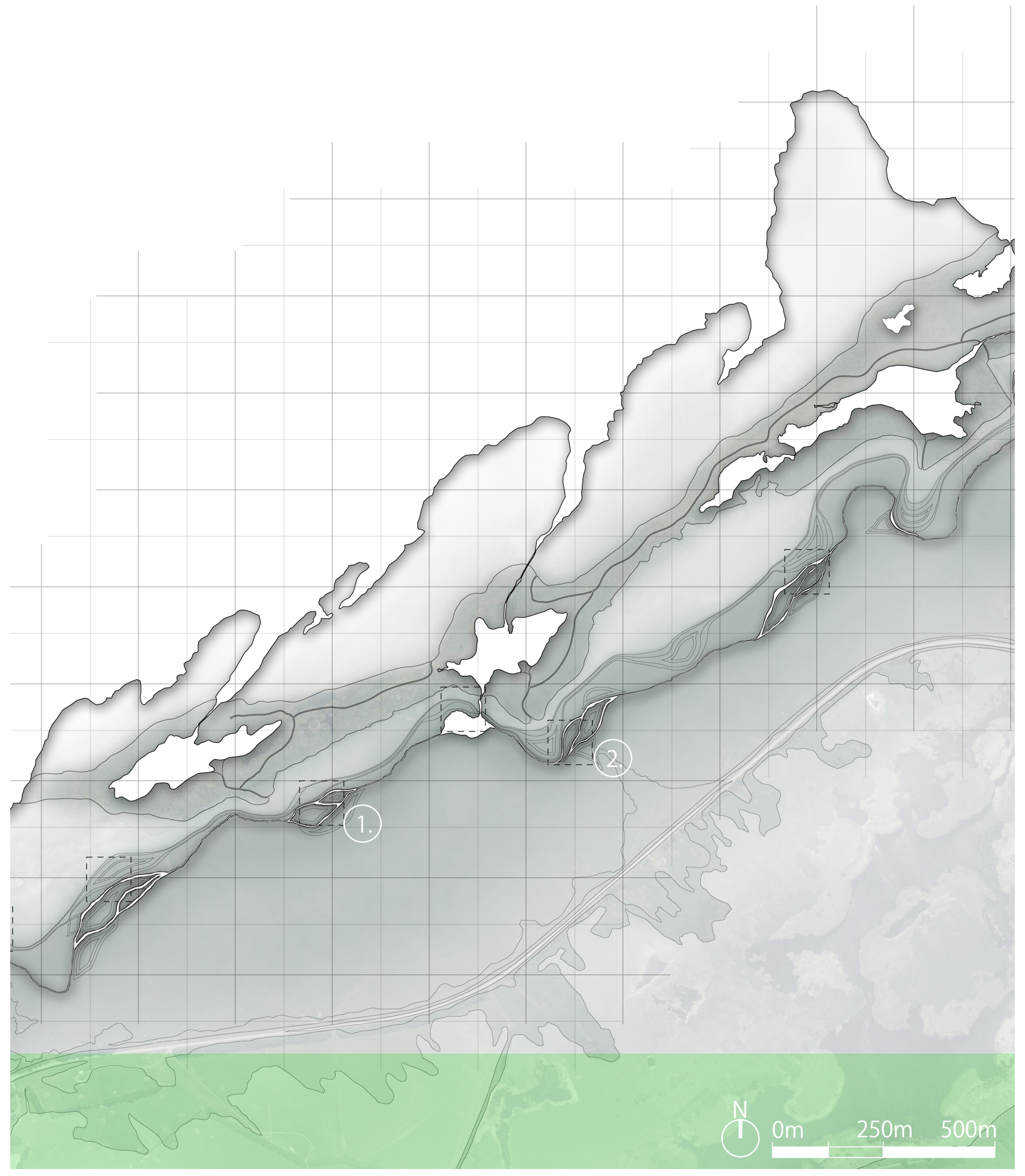



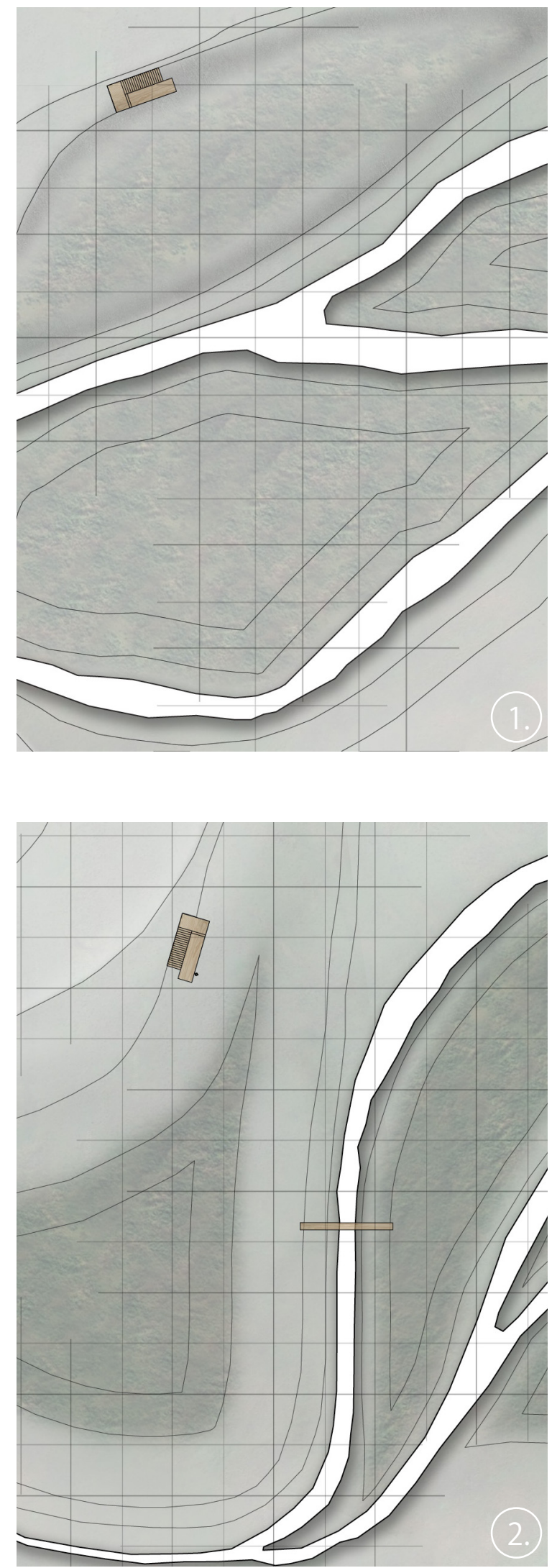

Reflecting of the multiuse space that they sit within, the view platforms also facilitate various functions for visitors. Situated with close proximity to access ways for kayaking, swimming and other water based recreation, each platform has an integrated clean water system (Fig 84.0). Offering outdoor showering facilities and the ability to clean off mud and dirt from the surrounding site. Each platform offers further positive interaction with the site water. Manually pumped, water from the nearest treated ponds enters the structure, a holding tank integrated into the platform ensures a suitable pressure is achieved through gravity. By people having to pump their own water into the holding tank, a respect for its immediate use as a volume is gained. Less water is to be wasted, or taken for granted when it has to be directly worked for. Small design moves such as this further inform people of the power water has within our natural environment. Especially the need to protect our waterways and larger hydrological systems. (Fig 84.0)

Fig 84.0 Site 2 view platform placement (Opposite) 

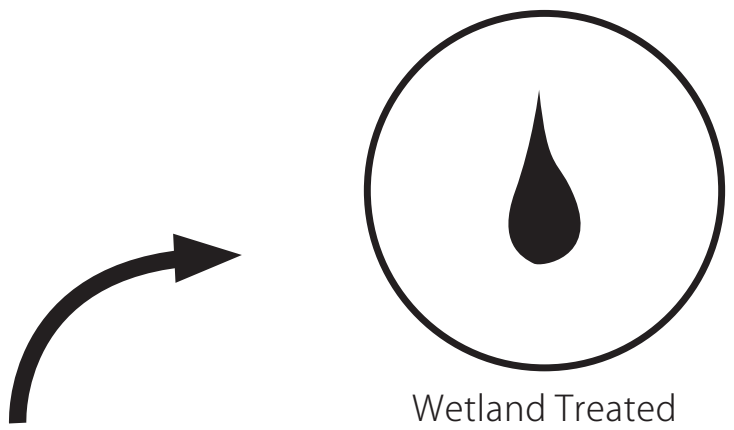

Wetland Treated Clean Water
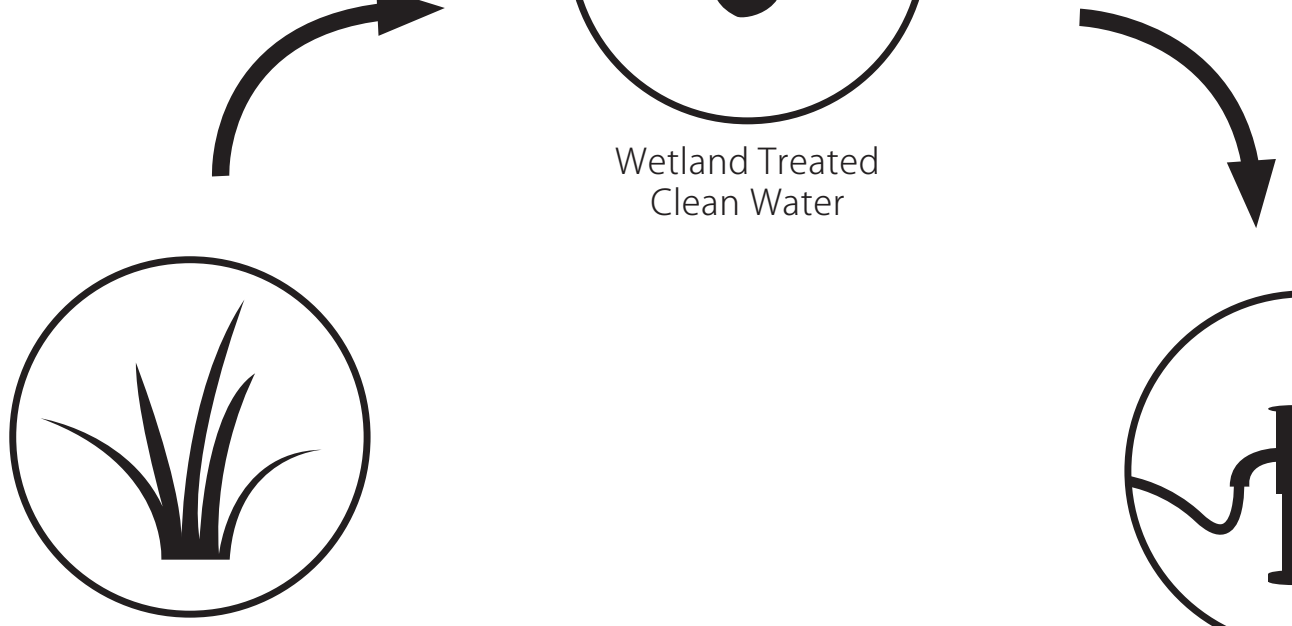

Wetlands treat water runoff

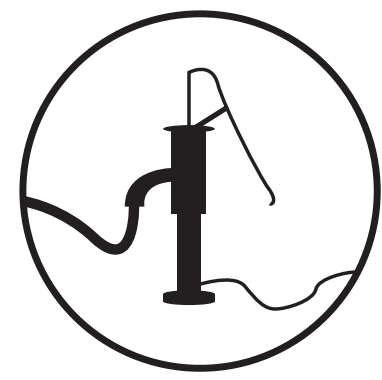

Hand-pumped as needed

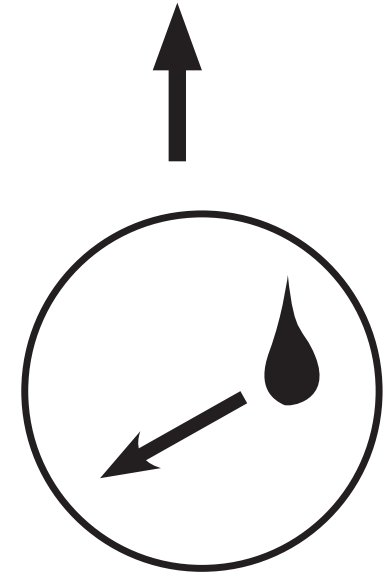

Water runs back to stream
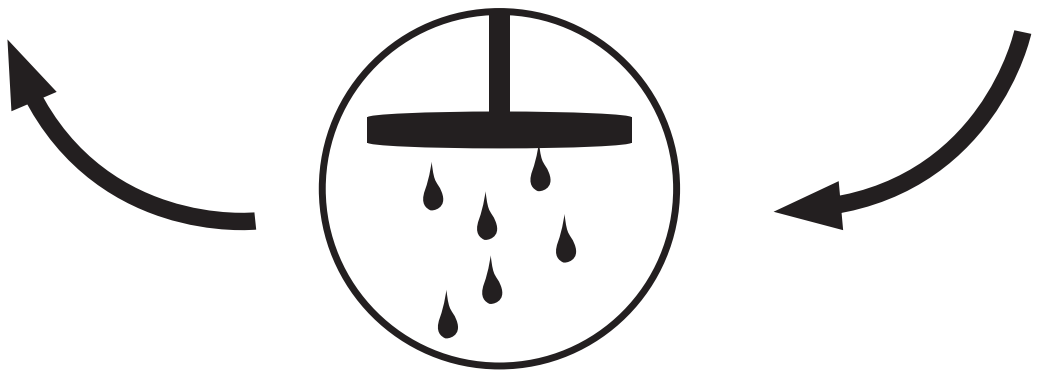

Gravity fed water shower

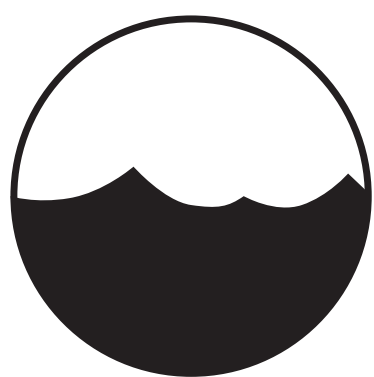

Fills holding cell in platform 
The main focus of the platform is to further exaggerate the height created by the wetland infrastructure in such a flat, horizontal landscape. By elevating people above, the waters journey from polluted to clean can be easily viewed. The full extent of the intervention is highlighted by the curves of the design in contrast to the harsh surrounding farmland and lake shore. The function of the water system means the platforms must have close proximity to the clean water ponds, but a direct view to the lake also (Fig 85.0). As one of the issues with the lakes water quality lies with suspended sedimentation, the pollution is visible even to the untrained eye. By allowing people to view this pollution, and its extent over the entire lake system, the problem is made blatantly obvious. Seeing our natural landscape in such a degraded form encourages visitors to take ownership of the problem and realise the importance of the space this design offers by also showing the contrasting clean water pond. 

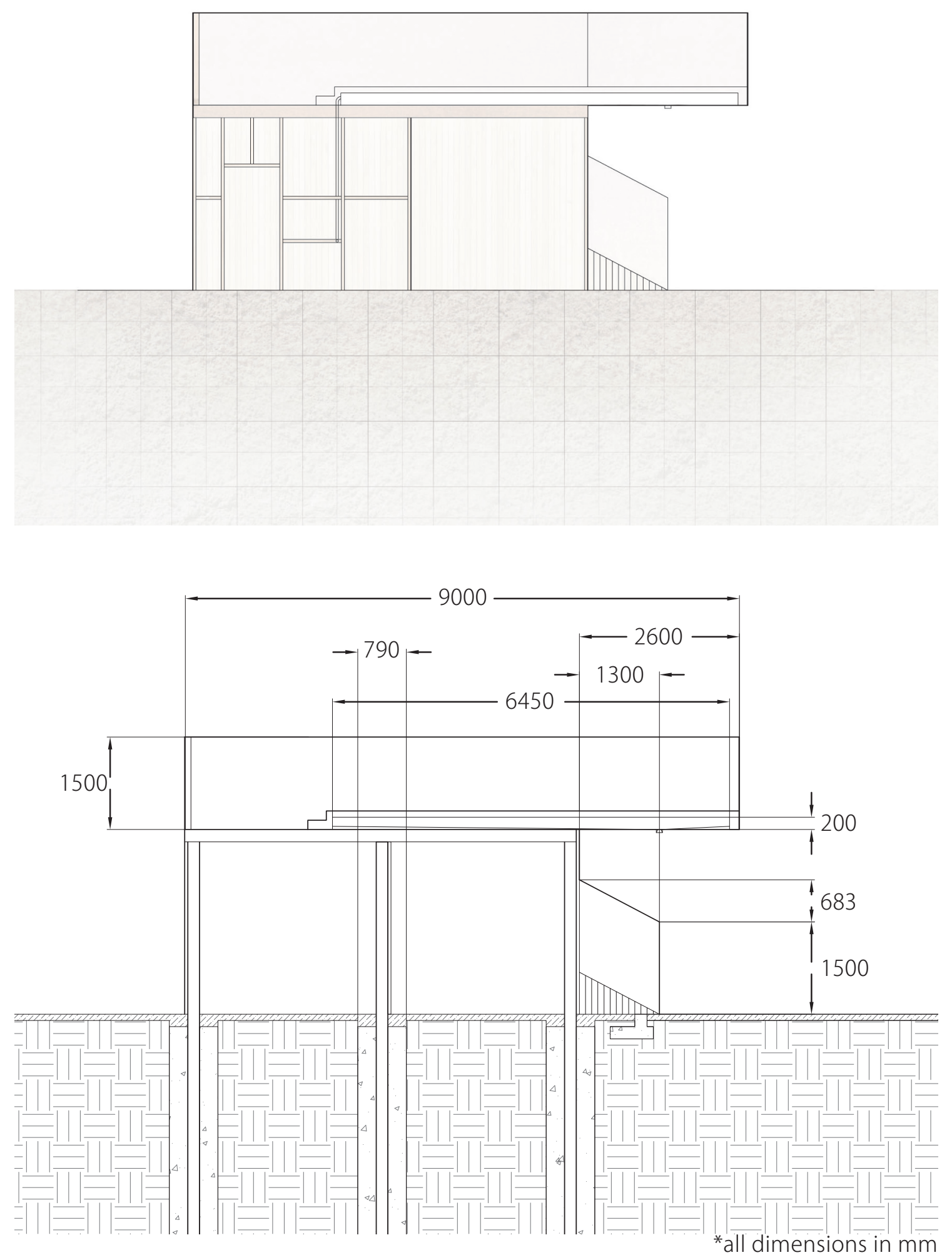


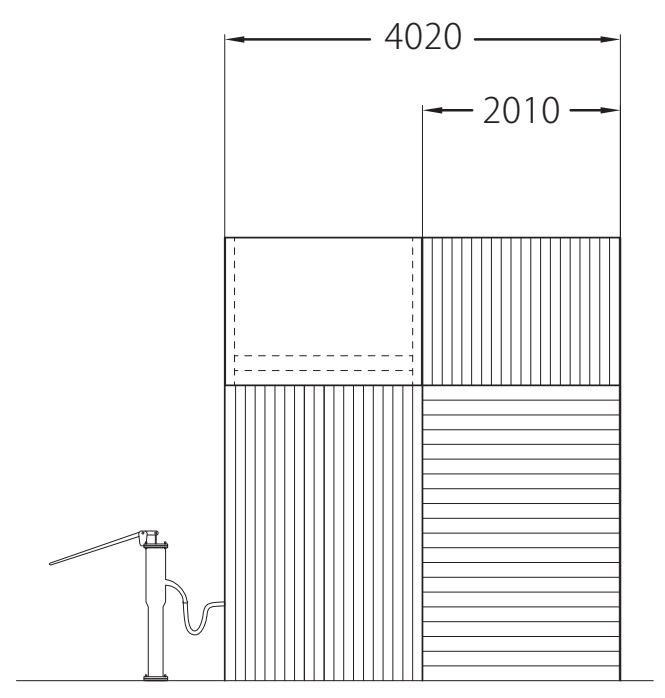

A simple feature of the design, the view platforms are a great part of making this site a unique landscape that will draw visitors (Fig 86.0). Lake Wairarapa is an invaluable natural resource that has the potential to be returned to its former use as a recreational hub. Providing for the needs of the landscape and the visitors this space is the first step in regaining recreation within Wairarapa Moana. Adding the unique element of height in this landscape also means a positive change over time can be viewed by people who continue to return to this design. Interaction with the landscape is imperative in encouraging people to take ownership for the problems Wairarapa Moana faces and move forward to reclaim their natural landscape.

Fig 86.0 View platform elevation

(Opposite, top)

Side elevation with cladding removed showing the changing room in the rear of the platform

Fig 87.0 View platform construction section

(Opposite bottom)

Fig 88.0 View platform end elevation

(Above) 

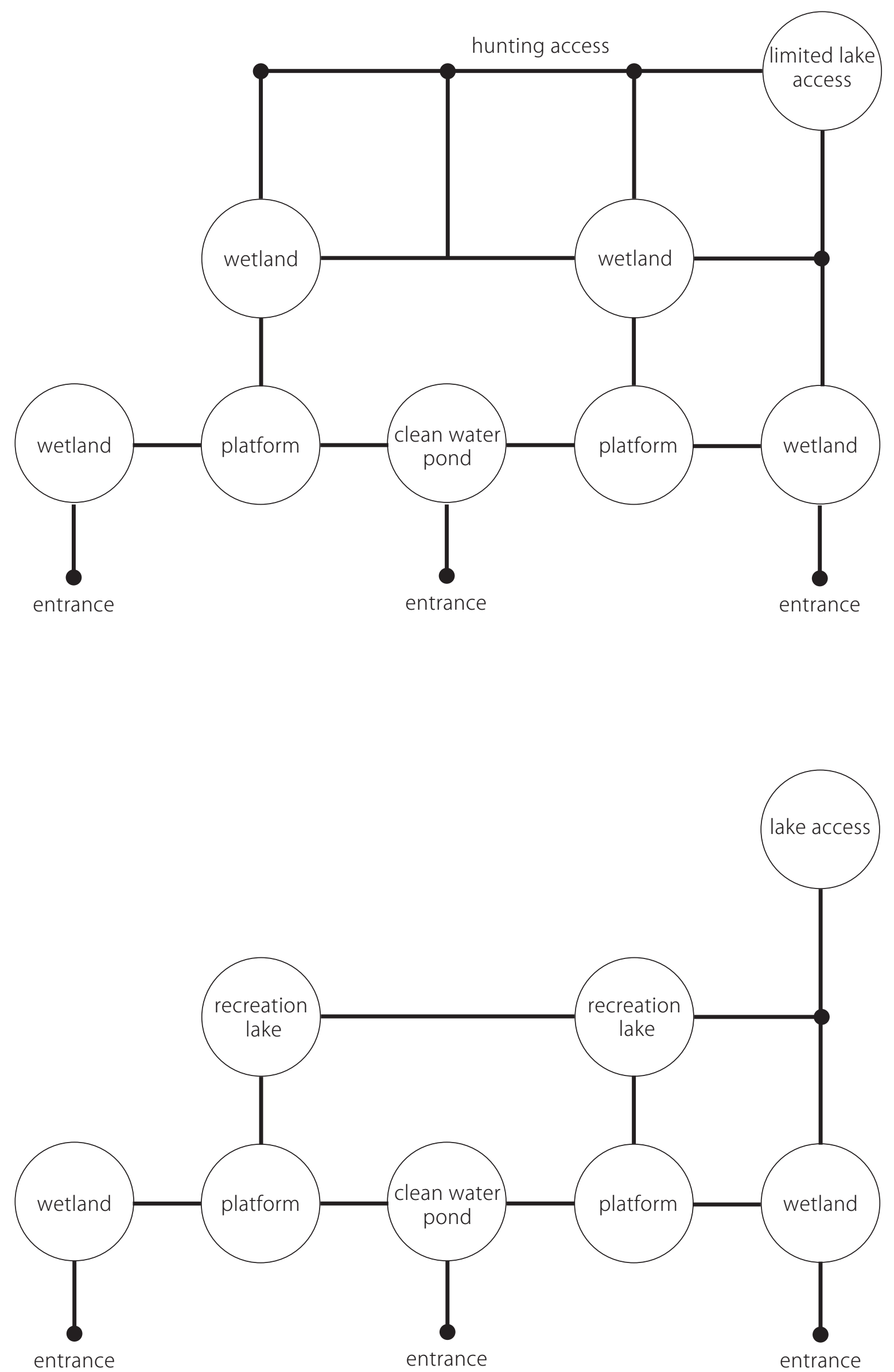


\subsubsection{THE SEASONAL SITE}

Approaching design from a seasonal perspective requires diversity to be at the core of every move. Wairarapa Moana's seasonal landscape is viewed as a feature, not a hindrance, in this design intervention. The seasonal change of weather is not the only dynamic element of this site, existing programme such as duck hunting and bird watching are also very reliant on seasons. (Fig 89.0) Catering for the needs of multiple users has resulted in a diverse space that responds to the needs of the different seasons. The design of the site also changes with the seasons creating a dynamic landscape that responds to needs of its users by offering multiple recreational activities. (Fig 90.0)

Building on existing programme of site was very important. Game hunting seasons vary from year to year, however the season is typically from May to August. Visitors to site are typically low during these months of a colder climate. Providing access to Maimai (Camouflaged huts for hunters to hide and shoot from) is necessary during this time. The result is a secondary access route available for hunters to use only during the hunting season, at other times during the year access is limited as navigation to these sites are seasonal and require a map for the unsuspecting visitor. Every effort to protect the natural landscape while still supporting recreation has been considered to achieve the most possible diversity within this design through the separation of hunting and family recreation by distinctive areas accessible only to those who obtain a hunting map of the area.
Fig 89.0 Site 2 hunting season access diagram (Opposite, top)

Fig 90.0 Site 2 typical access diagram (Opposite, bottom) 


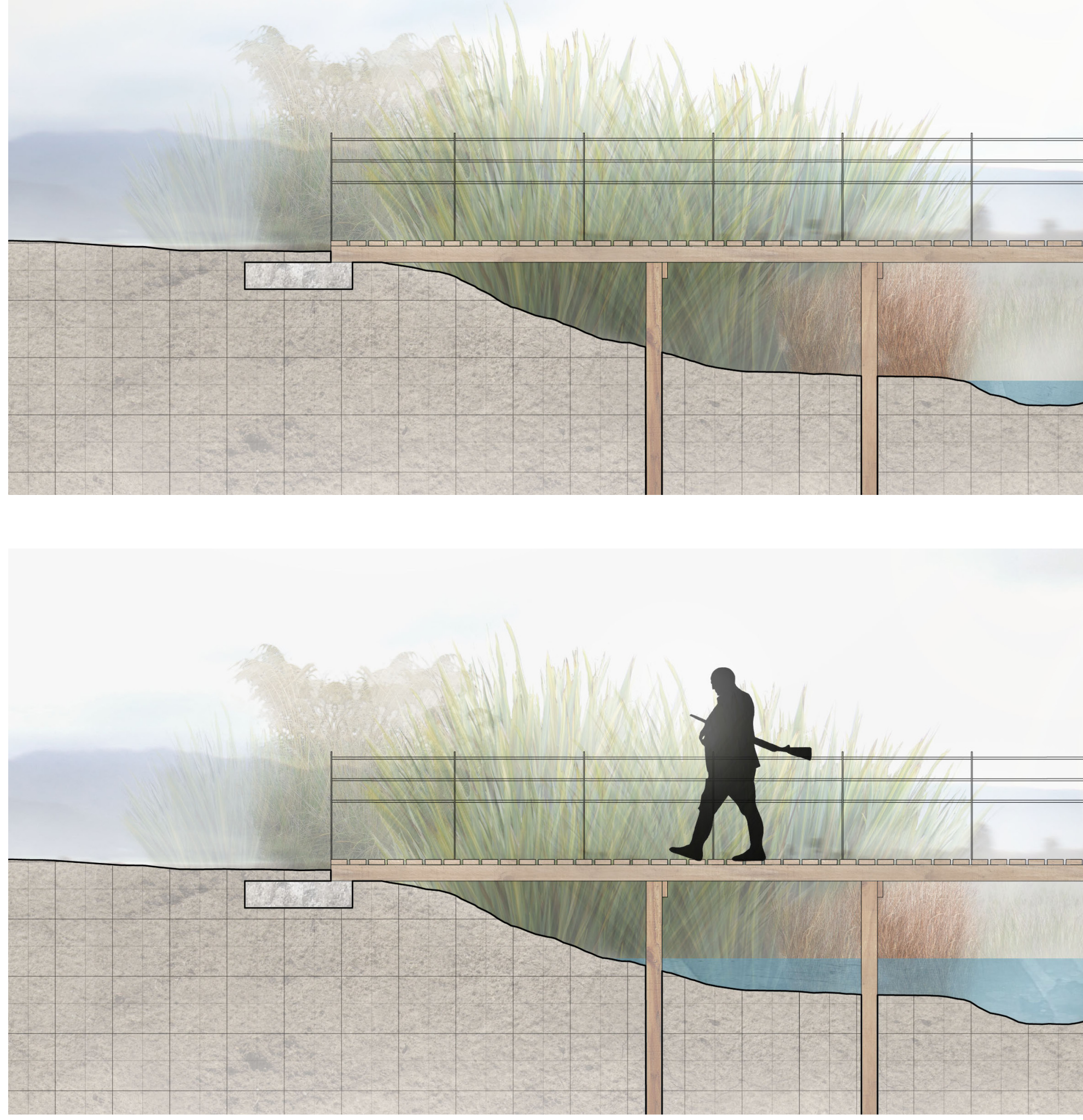


hunting access

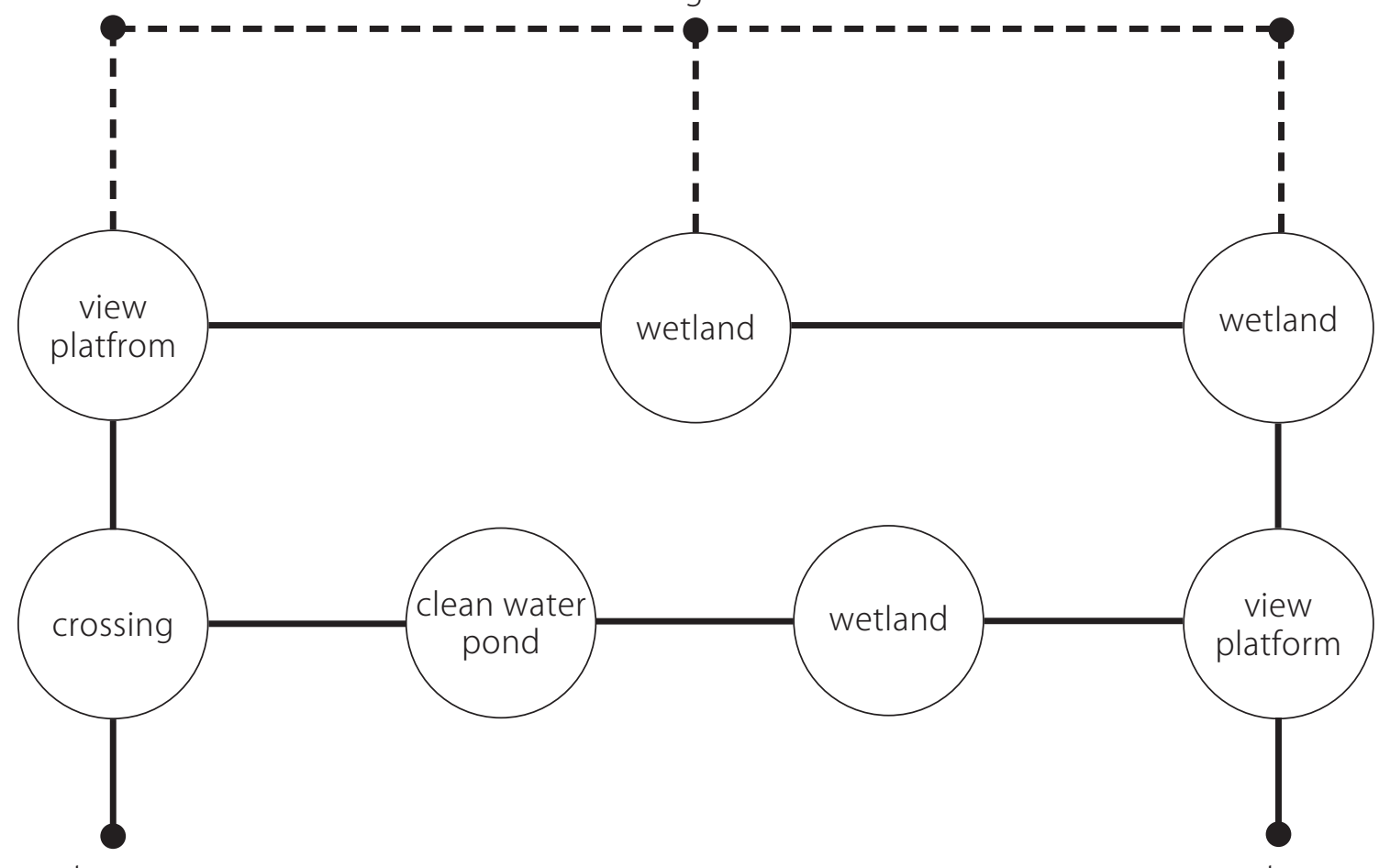

entrance

entrance

hunting access

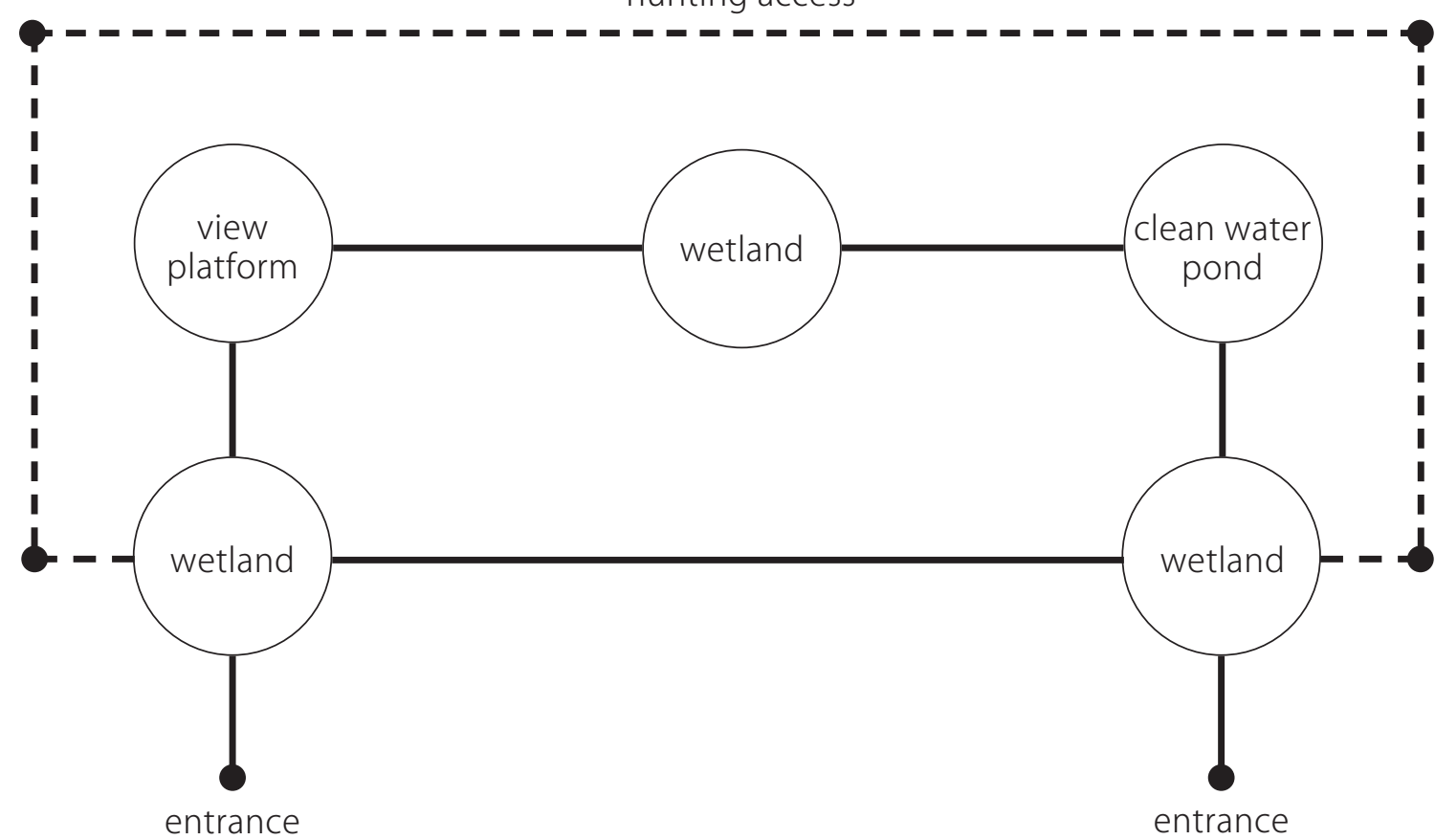


Alongside recreational seasons, the climate also has a significant role in how humans interact with the site. During the warmer season, water on site is not as abundant as it can be during the wet season. To accommodate this significant change in season, the wetlands are designed to function even in the heat of summer. The ability for these wetlands to adapt to the flux of the seasons on site ensures their success. Equally the visitors to the site will be much greater in the warmer season as there are more daylight hours, and the weather is more supportive of being outdoors. As the wetlands are not in flood during this time, full access and circulation routes are available to visitors, allowing a huge variation in how the site design can be navigated (Fig 93.0-94.0). Water retention is also a feature of the design through the central pond, any water that enters the site is cleaned as it makes its way towards this pond. The height of the discharge outlet back into the lake system results in water remaining at a clean, usable level, only discharging when a maximum level is met. The reverse function happens during winter, water is abundant on site meaning raised water levels and more frequent discharge back to the Lake. The ability of the site to react to seasons results in a landscape that can be used at any time throughout the year, meeting all needs of the people who enter the site.

The ability of a landscape to react to changing circumstances is a vital part of ensuring the longevity of the design. Meeting the dynamic needs of both people and the natural environment, this design is multifunctional in any season. Climatic and recreational programme have all been considered in the design to achieve a landscape that is an asset to the South Wairarapa region.

Fig 93.0 Site 1 access/circulation diagram Fig 94.0 Site 3 access/circulation diagram 


\section{DISCUSSION}




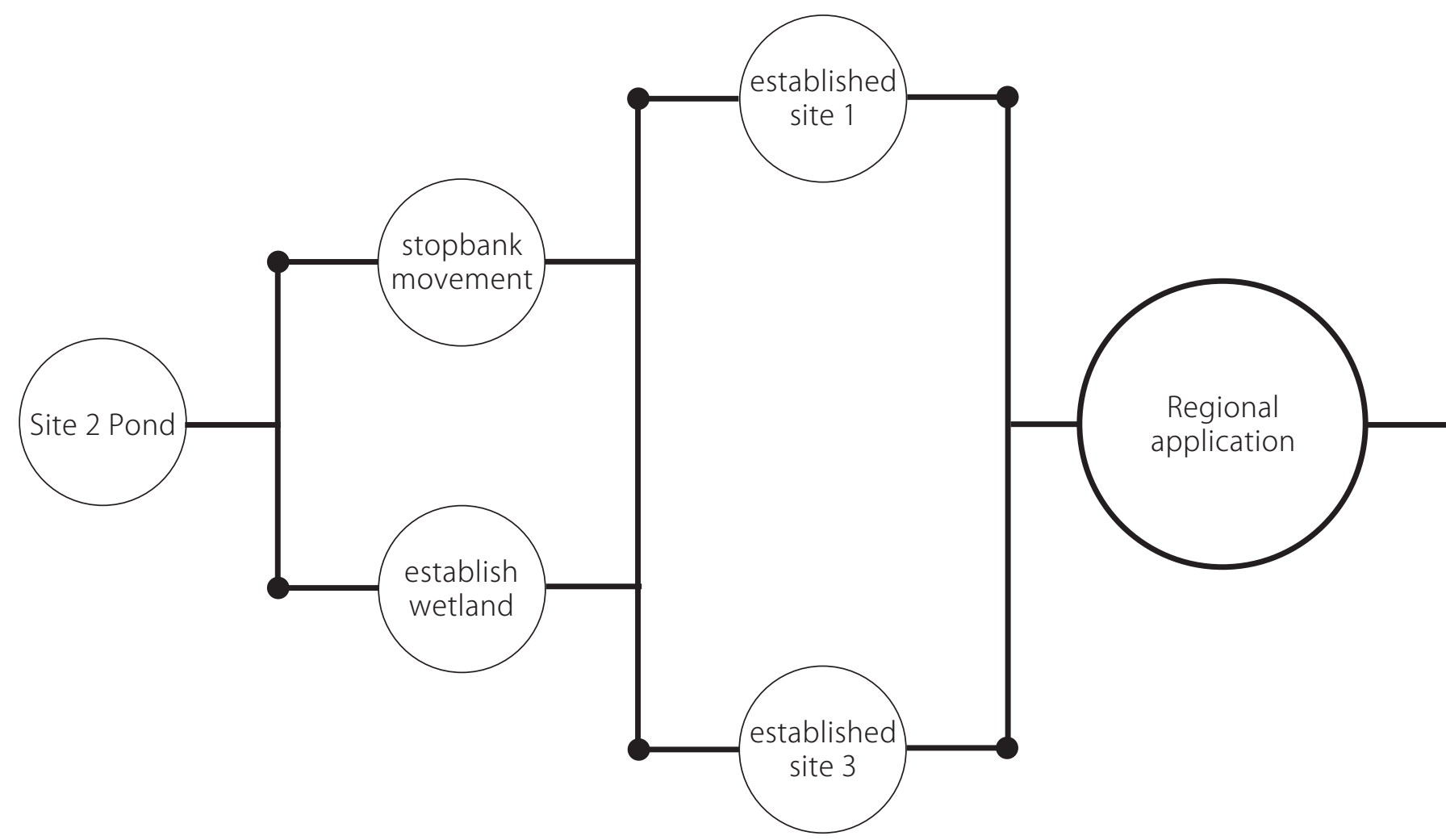




\subsection{A KINSHIP WITH THE LAND}

Regional landscapes create the backbone of New Zealand, made up of vast amounts of land that are home to sensitive ecosystems that support our natural environment. Settlement within these landscapes has traditionally seen heavy and detrimental infrastructure that scares the land. The spatial separation of ecology and infrastructure as a disciplinary tendency leaves a void that has potential for development to remediate our landscapes as well as the discipline of landscape architecture.

Precedent projects around the world have begun to explore infrastructure that is designed to fit seamlessly within its natural surroundings. However, those designs address problems commonly from an aesthetic and experiential approach which mainly deals with the power of the landscape's experience and the use of light infrastructure. However, the function of such infrastructure is still implemented in a traditional fashion. Advances within the discipline of landscape architecture

50 years

Fig 95.0 Design phasing timeline 
have recently allowed infrastructure to develop. Through mimicking natural systems of the landscape, a less invasive outcome can be achieved as it happens in the wetlands introduced in this design research.

The process of identifying relationships on site, as the first step, has lead this research to reach a new level of infrastructure design. One that is respectful of the native ecosystems creating a natural aesthetic that subconsciously builds New Zealand's cultural identity. This creates an environment with a natural feeling that benefits the landscape and the people who share the space. Identification of relationships between vegetation and human interference was part of a critical analysis that allowed the selection of the most appropriate sites to design. The design outcome is a minimally invasive typology due to its reliance on mimicking the natural systems and restoring back a long lost wetland system that now fully preserves the identity of the site. By combining infrastructure with open space, this typology accomplishes synergy between function and respect.
The core function of this design relies on water treatment and native wetlands. The manipulation of these wetlands to treat polluted water, as well as providing expanses of recreational space, directly benefits the wider ecosystem and the human inhabitants within it. The simultaneous design for people and the land has allowed a rich recreational program to be developed. People are drawn to the space for its recreational purposes which are not offered anywhere else within the region. This can be complimented by the design which offers a large range of activities.Ultimately the users can enjoy these amenities while discovering and learning about the natural system in place.

Each of the three sites successfully treat polluted lake water, having a local impact on the hydrological system. This sets the potential to be the catalyst for future designs at a regional scale, benefiting the wider ecosystem and the perception of the lake as a natural amenity. 
To gain impact at a regional scale, a large amount of land would be required to improve the quality of the hydrological system. This will contribute to enhance the water experience of visitors to Wairarapa Moana and therefore improve their perception of the region. This can be accomplished through a sequential implementation as expressed in the phasing time line, (Fig 95.0) where the same design principles can be embedded in a regional framework. 


\subsection{FINAL FINDINGS}

The need for our landscapes to be protected in the future through design is fundamental principle of landscape architecture as a discipline. However outside of landscape architecture few people share the same passion for its value. A shift in thinking is needed specifically design of infrastructure that would see more resilient design replacing the typically heavy approach that is currently commonplace.

To encourage a change in thinking that restores the value to the landscape for its natural amenity, this study approached water infrastructure in the South Wairarapa. Typically water treatment has harmful effects on the landscape as the infrastructure required for chemical treatment is large and harmful. The implementation of wetlands as water treatment infrastructure mean this harmful effect on the landscape has been removed instead it is replaced with a natural landscape that supports the recreation needs of local and visiting people.

Using landscape architecture to reconfigure public land, designed spaces that treat water and support many recreational programmes are achieved. Visitors to these spaces can enjoy the landscape for its amenity value as they make the most of their interaction with clean water on each site weather they are swimming in the fresh water ponds or kayaking the larger lakes in the surrounding areas.

While enjoying the recreation offered within the water treatment areas, visitors can also gain an education from the landscape. A visible change in water quality can be viewed as people move through each site, polluted water enters and clean water exits. An education on the importance of natural infrastructure and the value it has for our larger hydrological systems is easily realised.

Education and positive interaction with water are key drivers in the changing of peoples' perception of water in Wairarapa Moana. Often viewed as a negative space, the outcomes achieved in this research change the perception of water from negative to positive. By using natural systems to treat the currently poor water quality the space 
becomes popular for its recreational benefits, alongside its function as infrastructure. A change in perception of this space leads to more respect for the power of the natural landscapes within South Wairarapa, as a result New Zealand's unique cultural identity with the landscape is strengthened and protected for the future by including water infrastructure into public space. 


\section{BIBLIOGRAPHY}

Benedict, Mark A. and Edward T. McMahon. Green Infrastructure : linking landscapes and communities. Washington, DC: island Press, 2006. Print.

Corner, James. Recovering Landscapes, Essays in Contemporary Landscape Architecture. New York: Princeton Architectural Press, 1999. Print.

Corner, James. "Terra Fluxus." Waldheim, Charles. The Landscape Urbanism Reader. New York: Princeton architectural Press, 2006. 022-033. Print.

Crowe, Sylvia, and Zvi Miller. Shaping Tomorrow's Landscape. Amsterdam: Djambatan, 1964. Print.

DOC, Environment Bay of Plenty, and Fish \& Game New Zealand. Wetland Restoration Guide. N.p.: Bay of Plenty Wetlands Forum, n.d. PDF.

France, Robert L. Wetland Design: Principles and practices for landscape architects and land-use planners. New York: W. W. Norton, 2003. Print.

Landezine. Landezine:The Brick Pit Ring by Durbach Block Architects . 30 January 2012. Web page. 25 September 2015.

Moore, Kathryn, Prof. "HS2 Can Be Beautiful - Not a Blot on the Landscape." Birmingham City University. N.p., 15 July 2013. Web. 10 July 2015. < http://blogs.bcu.ac.uk/ views/2013/07/15/hs2-can-be-beautiful-not-a-blot-on-the-landscape/>

Peart, Raewyn. A Place to Stand: The Protection of New Zealand's Natural and Cultural Landscapes. Auckland, N.Z.: Environmental Defence Society, 2004. Print. 
Perrie, A and J R Milne. Lake water quality and ecology in the Wellington region: State and trends. Water quality monitoring report. Wellington: Greater Wellington Regional Council, 2012. PDF. <http://www.gw.govt.nz/assets/councilpublications/SoE\%20-\%20Lake-water-quality-and-ecology-\%20\%28abstract $\% 20$ in\%20doc\%29.pdf>.

Sherwood, Reed C. Subsurface Flow Constructed Wetlands for WasteWater Treatment. N.p.: United States Environmental Protection Agency, July 1993. PDF.

Statistics New Zealand. "South Wairarapa District, Population and Dwellings." Statistics New Zealand, 2013. Web. May 2015. < http://www.stats.govt.nz/Census/2013census/profile-and-summary-reports/quickstats-about-a-place.aspx?request_ value $=14536 \&$ tabname $=>$

Te Whaiti, Haami, et al. Wairarapa Moana, The lake and its People. Ed. Ian F. Grant. Masterton: Wairarapa Archive, 2012. Print.

Waldheim, Charles. The Landscape Urbanism Reader. New York: Princeton Architectural, 2006. Print.

Wall, Alex. "Programming the Urban Surface." Corner, James. Recovering Landscapes, Essays in Contemporary Landscape Architecture. New York: Princeton Architectural Press, 1999. 233-249. Print.

.id. South Wairarapa District. 2013. 2015 й и л 05-December. <http://profile.idnz. co.nz/greater-wellington/about?Web|D=150>. 


\section{LIST OF FIGURES}

Figure 14.

http://www.gw.govt.nz/assets/Parks-and-Recreation/Wairarapa-Moana/wairarapamoana-wetlands-park-brochure.pdf

Figure 17.0

http://www.ccc.govt.nz/assets/Documents/Environment/Water/

SWPAvonCatchmentCCC.pdf

Figure 18.0

https://ccdu.govt.nz/projects-and-precincts/te-papa-\%C5\%8Dt\%C4\%81 karoavonriver-precinct

Figure 19.0

https://www.google.co.nz/maps/@-43.5288053,172.6624455,13.5z?hl=en

Figure 21.0

http://www.landezine.com/wp-content/uploads/2012/01/01-DurbachBlock-photoby-Peter-Hyatt.jpg

Figure 23.0

https://www.google.co.nz/maps/@-33.8427986,151.0684822,4958m/ data $=! 3 \mathrm{~m} 1 ! 1 \mathrm{e} 3$ ?hl=en 
Figure 25.0

http://www.landezine.com/index.php/2010/10/trollstigplataet/reiulf_ramstad_ trollstigplataet_norway_03/

Figure 26.0

http://www.landezine.com/index.php/2010/10/trollstigplataet/reiulf_ramstad_ trollstigplataet_norway_08/

Figure 27.0

https://www.google.co.nz/maps/@62.1277447,7.1729612,7518m/

data $=! 3 \mathrm{~m} 1 ! 1 \mathrm{e} 3 ? \mathrm{~h} \mid=\mathrm{en}$

All figures not referenced are authors own. 


\section{APPENDIX}

Appendix 1.0 Lake Wairarapa wetland hydrologic loading estimate (Opposite)

Thompson M and Mzila D. 2015. Lake Wairarapa water balance investigation: Stage 1 report - interim findings and recommendations. Greater Wellington Regional Council, Publication No. GW/ESCl-T-15/47, Wellington. <http://www.gw.govt.nz/assets/ council-publications/Lake-Wairarapa-Water-Balance-Stage-1-Report.pdf> 


\section{Conclusions and recommendations}

A water balance model for Lake Wairarapa has been developed and successfully calibrated for the summer of 2012/13. Summer allocation scenarios have been tested and the likely impacts on lake levels quantified. While further work is needed to translate the findings into firm conclusions regarding environmental consequence, it is considered that enough has been learned to inform recommendations about allocation management for Proposed Natural Resources Plan. These recommendations, including an interim allocation limit, are set out below.

\subsection{Recommended interim allocation limit for lake}

It is recommended that the interim limit for allocation of water directly from Lake Wairarapa (and its marginal drains) is $0.6 \mathrm{~m}^{3} / \mathrm{s}$ or $39,635 \mathrm{~m}^{3} /$ day. This equates to the existing (as at 15 November 2014) level of consented allocation from the lake and therefore represents a cap on any new takes. It also implies that the interim limit for allocating 'lake depleting' water from tributaries and groundwater in the wider lake catchment should equate to the existing level of consented allocation $\left(1.8 \mathrm{~m}^{3} / \mathrm{sec}\right)$.

The limit is informed by the preliminary findings in this report that the lake water level is likely to be relatively sensitive to increases in allocation; small changes in volume can potentially lead to quite large shoreline exposures. The recommended limit is not based on an explicit assessment of the environmental consequence of shoreline recessions due to increased allocation. It simply reflects the line of reasoning that any increase in allocation would be difficult to justify given the existing difficulty that is sometimes experienced in meeting summer minimum target levels, the likely sensitivity of the lake levels to abstraction and the lack of information about environmental consequences. It is considered prudent to adopt a precautionary approach until alternative allocation scenarios can be debated in the context of overall Lake Wairarapa management.

\subsection{Recommended management of existing abstractions}

In addition to setting an allocation limit, it is recommended that existing water takes are managed to prevent them exacerbating lake level reductions in times of summer water stress.

Currently, consents to take water directly from the lake are subject to cease take conditions when the lake is below target minimum levels. However, these conditions leave water users with a very low security of supply over summer months because the lake is so frequently below target levels (on average, 65 days per summer). This situation, combined with some doubt as to whether being below target levels represents a genuine period of water stress, has led to GWRC exercising discretion as to whether cease take conditions are enforced.

A change in approach is recommended to provide more certainty. Rather than being based on the single premise of maintaining a minimum target level, it is recommended that existing takes be restricted or required to cease in accordance with the status of all of the following: 
Appendix 2.0 Lake Wairarapa wetland hydrologic loading input water level (Opposite) Thompson M and Mzila D. 2015. Lake Wairarapa water balance investigation: Stage 1 report - interim findings and recommendations. Greater Wellington Regional Council, Publication No. GW/ESCl-T-15/47, Wellington. <http://www.gw.govt.nz/assets/ council-publications/Lake-Wairarapa-Water-Balance-Stage-1-Report.pdf> 

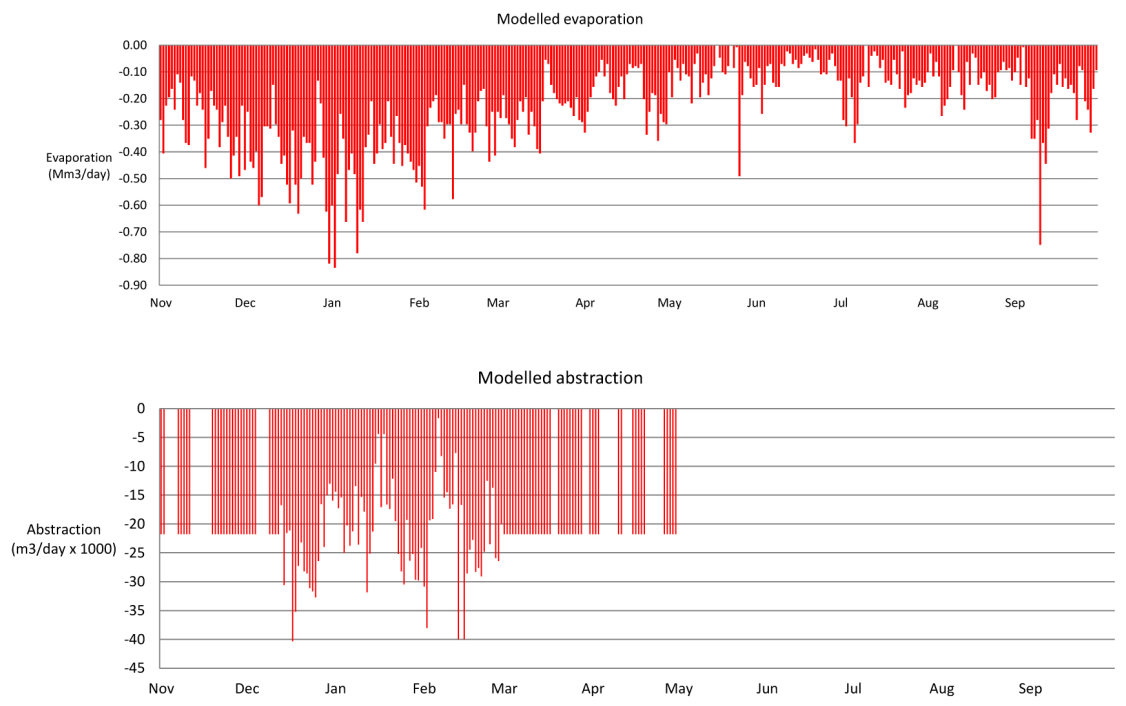

Figure 5.1 cont: Estimated daily time series for evaporation (top graph) and abstraction (bottom graph)

\subsubsection{Monthly water balance}

Table 5.1 shows the initial results for the monthly total flux for the primary water balance components for the period November 2012 to September 2013.

Table 5.1: Lake Wairarapa monthly total inflows and outflows (Millions of $\mathrm{m}^{3}$ ) for the period 01 November 2012 to 30 September 2013

\begin{tabular}{|c|c|c|c|c|c|c|c|c|c|c|c|c|}
\hline & Nov & Dec & Jan & Feb & Mar & Apr & May & Jun & Jul & Aug & Sep & $\begin{array}{l}\text { Seasonal } \\
\text { pattern }\end{array}$ \\
\hline Rainfall & 3.3 & 6.5 & 4.7 & 5.6 & 8.1 & 7.0 & 10.5 & 16.6 & 5.8 & 5.4 & 9.7 & \\
\hline Stream inflow ${ }^{1}$ & 14.9 & 27.7 & 28.4 & 13.1 & 19.4 & 28.6 & 40.8 & 94.8 & 68.3 & 53.3 & 98.8 & \\
\hline Barrage Inflow & 4.6 & 8.5 & 0.1 & 3.4 & 12.7 & 4.7 & 18.0 & 4.7 & 1.5 & 4.7 & 6.8 & 1 \\
\hline Barrage Outflow & -20.7 & -11.8 & -14.8 & -7.4 & -31.1 & -16.1 & -46.2 & -125.9 & -122.5 & -53.9 & -66.3 & - \\
\hline Evaporation & -8.3 & -13.1 & -13.7 & -8.9 & -7.6 & -5.3 & -3.5 & -2.6 & -4.4 & -3.9 & -6.2 & \\
\hline Abstraction & -0.4 & -0.7 & -0.6 & -0.6 & -0.6 & -0.3 & 0.0 & 0.0 & 0.0 & 0.0 & 0.0 & IIII" \\
\hline $\begin{array}{l}\text { Balance } \\
\text { [modelled } \\
\text { storage change } \\
+ \text { error] }\end{array}$ & -6.6 & 17.1 & 4.0 & 5.2 & 0.9 & 18.5 & 19.6 & -12.5 & -51.2 & 5.6 & 42.9 & \\
\hline $\begin{array}{l}\text { Actual storage } \\
\text { change }^{2}\end{array}$ & -5.9 & -0.5 & 1.0 & 2.5 & 0.6 & 10.9 & 5.0 & 13.3 & -16.5 & -0.9 & 20.2 & \\
\hline
\end{tabular}

${ }^{1}$ Stream inflow includes constant assumed groundwater inflow of $0.4 \mathrm{~m}^{3} / \mathrm{sec}$ or about $1 \mathrm{Mm}^{3}$ per month throughout the year

${ }^{2}$ Monthly storage change calculated from observed lake level and depth-dependent lake volume data. 
Appendix 3.0 Wetland functionality calculations based on formula found in this doccument

Sherwood, Reed C. Subsurface Flow Constructed Wetlands for WasteWater Treatment. N.p.: United States Environmental Protection Agency, July 1993. PDF. < http://www. ceadu.org.uy/documentos/Manual\%20EPA\%20de\%20Sistemas\%20Naturales.pdf> 
Since the term LW in equation (5) is equal to the surface area of the bed, rearrangement of terms in equation (5) permits the calculation of the surface area $(A$, required to achieve the necessary level of $\mathrm{BOD}_{5}$ removal:

$$
A_{s}=(L)(W)=\frac{Q\left[\ln \left(C_{0} / C_{e}\right)\right]}{K_{T} d n}
$$

(6)

$$
\text { Where: } \quad \begin{aligned}
A_{s} & =\text { bed surface area, } \mathrm{m}^{2}\left(\mathrm{ft}^{2}\right) \\
& \text { Other terms defined previously }
\end{aligned}
$$

The depth of media selected will depend on the design intentions for the system. If the vegetation is intended as a major oxygen source for nitrification in the system, then the depth of the bed should not exceed the potential root penetration depth for the plant species to be used. This will ensure availability of some oxygen throughout the bed profile, but may require management practices which assure root penetration to these depths. Table 6 presents results from the pilot system in Santee, CA (4) showing the relationship between root penetration and performance. The root depths shown in Table 6 are considered to be near the maximum practical limit to be expected. The design approach in Europe has also assumed a maximum depth of 0.6

\begin{tabular}{|c|c|c|c|c|}
\hline \multirow{2}{*}{$\begin{array}{l}\text { Bed } \\
\text { Type }\end{array}$} & \multirow{2}{*}{$\begin{array}{c}\text { Root } \\
\text { Depth, m }\end{array}$} & \multicolumn{3}{|c|}{ Final Effluent Quality, $\mathrm{mq} / \mathrm{L}^{\mathrm{a}}$} \\
\hline & & $\mathrm{BOD}_{5}$ & TSS & $\mathrm{N} \mathrm{H}_{3}$ \\
\hline Bulrush, Scirpus & 0.8 & 5 & 4 & 2 \\
\hline Reeds, Phragmites & 0.6 & 22 & 8 & 5 \\
\hline Cattails, Typha & 0.3 & 30 & 6 & 18 \\
\hline No Vegetation & 0.0 & 36 & 6 & 22 \\
\hline
\end{tabular}
$\mathrm{m}$ for Phragmites (15).

Table 6. Performance of Vegetated and Unvegetated SF Wetland Beds (4)

a. Primary effluent input $\left(\mathrm{BOD}_{5}=118 \mathrm{mg} / \mathrm{L}, \mathrm{SS}=57 \mathrm{mg} / \mathrm{L}, \mathrm{NH} 3=25 \mathrm{mg} / \mathrm{L}\right)$

There is one operational system in the U.S. (Monterey, VA) with a media depth

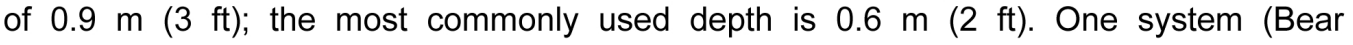




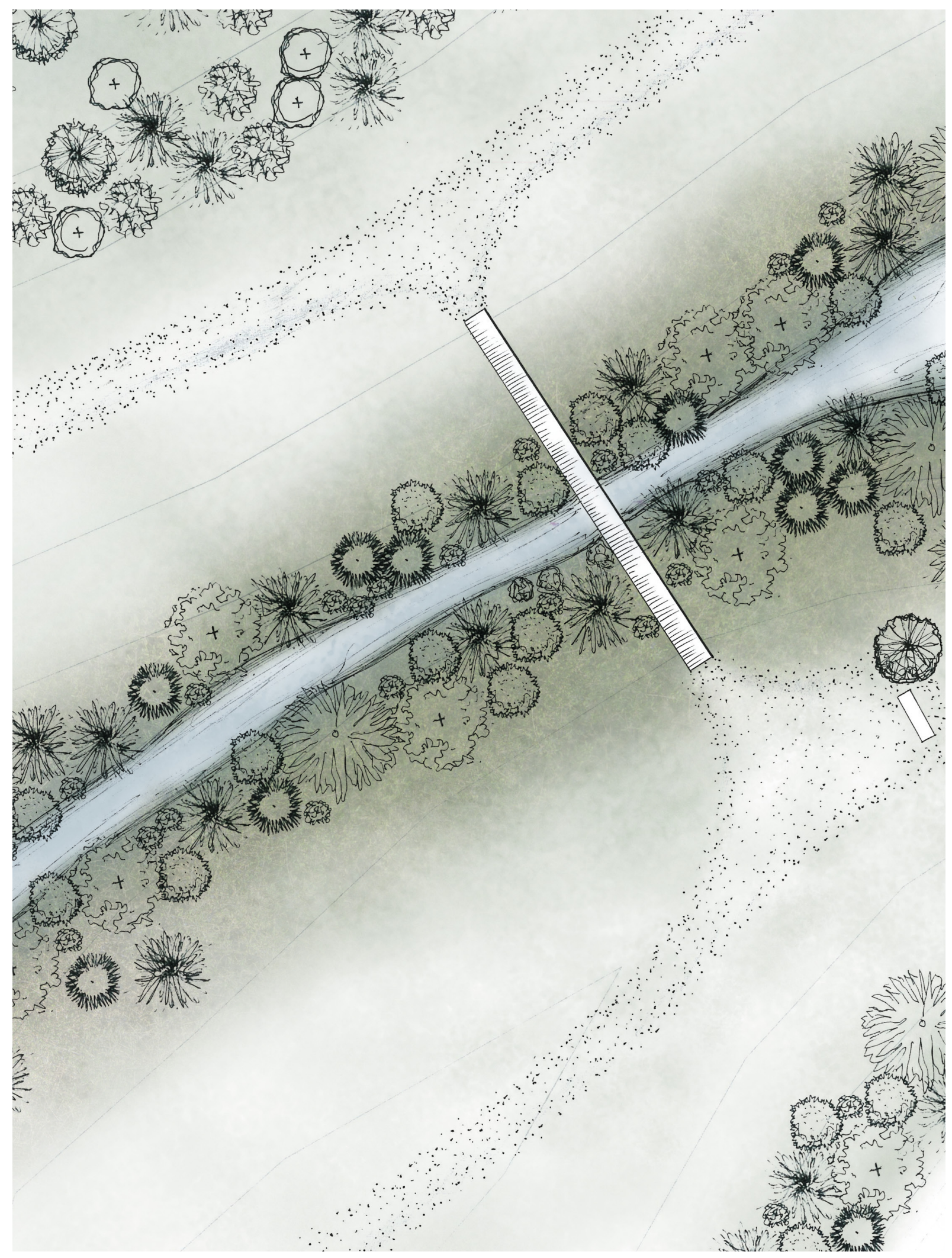

Appendix 4.0 Planting Plan site 3 typical low planting palette 1-200 scale at A4 


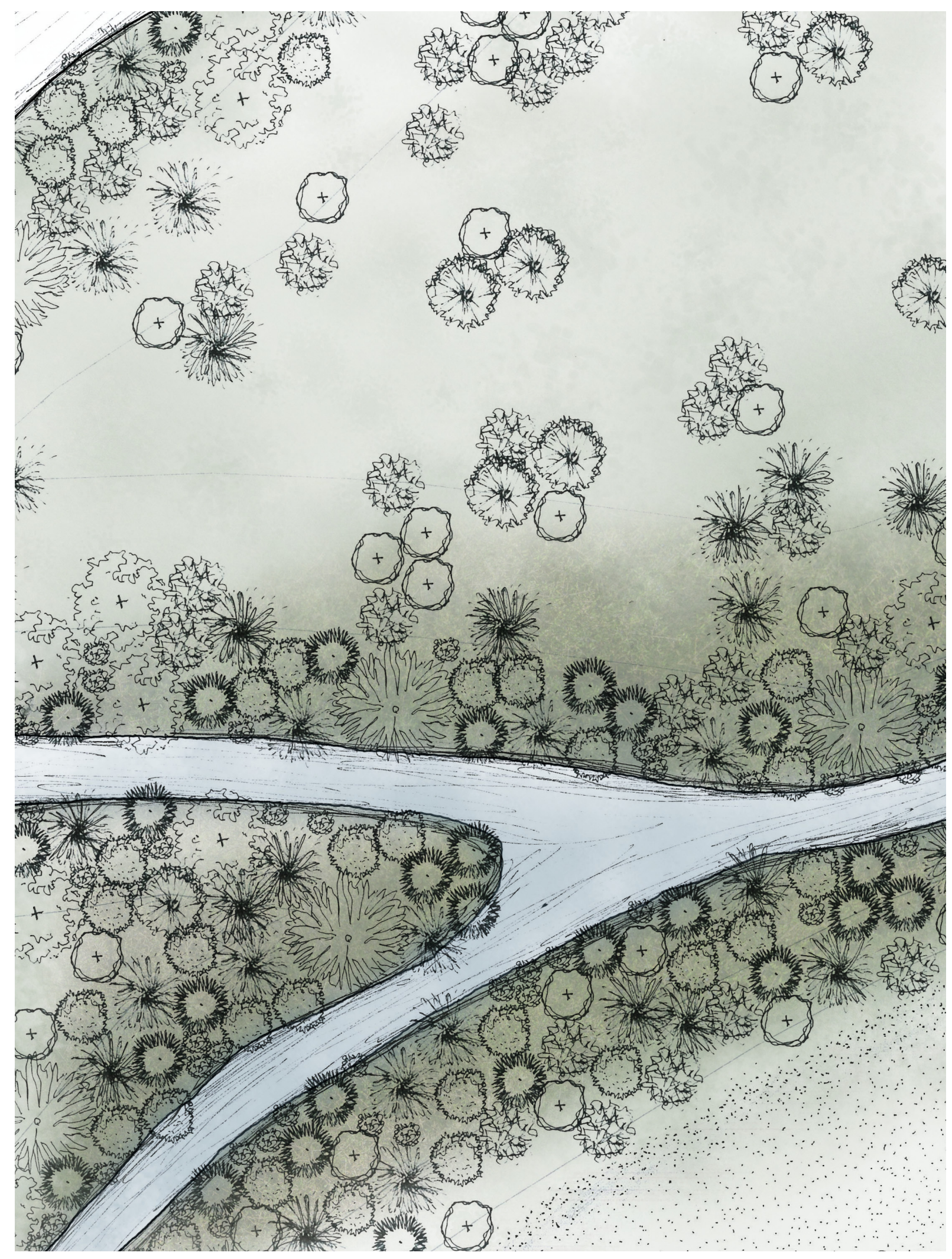

Appendix 5.0 Planting Plan site 3 typical stream planting palette 1-200 scale at A4 


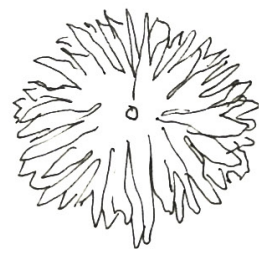

Typha orientalis (Raupo)

Spread: $5.0 \mathrm{~m}$

Height: $2.8 \mathrm{~m}$

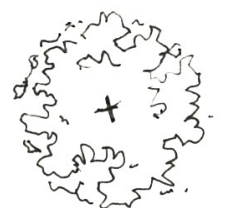

Syzygium maire (Swamp Maire)

Spread: $4.0 \mathrm{~m}$

Height: $8.0 \mathrm{~m}$

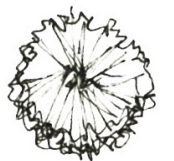

Cordyline australis (Cabbage Tree)

Spread: $3.0 \mathrm{~m}$

Height: $8.0 \mathrm{~m}$

Phormium tenax (Harakeke)

Spread: $3.0 \mathrm{~m}$

Height: $3.0 \mathrm{~m}$

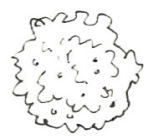

Cortaderia fulvida (Toe Toe)

Spread: $2.0 \mathrm{~m}$

Height: $1.5 \mathrm{~m}$

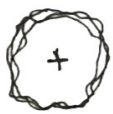

Leptospermum scoparium (Manuka)

Spread: $2.0 \mathrm{~m}$

Height: $4.0 \mathrm{~m}$

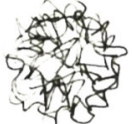

Coprosma propinqua (Mingimingi)

Spread: $2.0 \mathrm{~m}$

Height: $3.0 \mathrm{~m}$

Schoenoplectus tabernaemontani (Bulrush)

Spread: $2.0 \mathrm{~m}$

Height: $1.2 \mathrm{~m}$

Baumea articulata (Jointed twig-rush)

Spread: $2.0 \mathrm{~m}$

Height: $1.8 \mathrm{~m}$

Carex buchananii (Buchanan's sedge)

Spread: $0.8 \mathrm{~m}$

Height: $1.0 \mathrm{~m}$ 
